PROSPECTION AND GOALS IN HEALTHY AGING

\author{
By \\ Leann K. Lapp \\ Bachelor of Science, McGill University, 2007 \\ Master of Science, University College London, 2008 \\ Master of Science, Université Pierre et Marie Curie (Paris VI), 2009 \\ Master of Arts, Ryerson University, 2013

\begin{abstract}
A dissertation
presented to Ryerson University

in partial fulfillment of the requirements for the degree of

Doctor of Philosophy

in the Program of Psychology
\end{abstract}

Toronto, Ontario, Canada 2017

Copyright by Leann K. Lapp (2017) @ 


\section{AUTHOR'S DECLARATION FOR ELECTRONIC SUBMISSION OF A DISSERTATION}

I hereby declare that I am the sole author of this dissertation. This is a true copy of the dissertation, including any required final revisions, as accepted by my examiners.

I authorize Ryerson University to lend this dissertation to other institutions or individuals for the purpose of scholarly research.

I further authorize Ryerson University to reproduce this thesis or dissertation by photocopying or by other means, in total or in part, at the request of other institutions or individuals for the purpose of scholarly research.

I understand that my dissertation may be made electronically available to the public. 


\title{
PROSPECTION AND GOALS IN HEALTHY AGING
}

\author{
Doctor of Philosophy
}

2017

\section{Leann K. Lapp}

Psychology

Ryerson University

\begin{abstract}
The focus of this dissertation is on future prospection and goals in healthy aging. More broadly, this dissertation is contextualized within a particular theoretical framework that proposes that some of the cognitive decline associated with aging reflects adaptive changes in motivation and resource allocation (Hess, 2014). We used prospection as the cognitive domain within which to explore these motivational changes. In particular, we looked at a subtype of prospection known as episodic future thinking, which is concerned with how individuals imagine future scenarios, and what effects this might have on behaviour. Whereas the first experiment took up questions about motivational influences on the nature and bounds of future thinking deficits in older adults, the second experiment tested hypotheses about the adaptive function of future thinking. Specifically, episodic future thinking is often presumed to be adaptive for planning and successful action execution. Using prospective memory as a platform, we tested the idea that episodic future thinking can facilitate goal achievement, specifically in older adults.

In the first experiment, a cue-word paradigm, with words chosen to reflect age-relevant goal domains, was used to prompt the imagination of future scenarios. The results from the first experiment suggest that contrary to predictions, goal activation does not affect level of episodic detail in future thinking. However, phenomenological characteristics were modulated, with
\end{abstract}


younger adults showing more sensitivity than older adults. In the second experiment, participants employed different cognitive strategies - one of which included future thinking - in a gold standard test of prospective memory. The results from the second experiment failed to find episodic future thinking a helpful strategy and did not replicate previous work demonstrating a beneficial effect of another commonly used strategy, implementation intentions, which was used for comparison. Nonetheless, secondary analyses suggest that individual differences in strategy preference may be critical to consider before ruling out the utility of episodic future thinking. The results from these experiments contribute to a growing literature on motivation-cognition interactions across the lifespan, and suggest promising future directions regarding research into motivation, prospection, and healthy aging. 


\section{Acknowledgements}

Deep thanks are given first and foremost to my supervisor Dr. Julia Spaniol. A rigorous academic with the perfect measure of warmth, support, and flexibility, Dr. Spaniol was a pleasure to work with and learn from.

Additional thanks are given to my committee members Dr. Todd Girard and Dr. Lixia Yang, both of whom I've had the opportunity to work with in various capacities throughout my graduate training. They are highly respected members of our faculty and I was fortunate to benefit from their insights and support.

I owe thanks to the undergraduate students who helped me with my data collection: Stephanie Yung, Ryan Williams, and Ryan Marinacci. They are each talented individuals with promising futures ahead.

Finally, thanks to my family and AO, all of whom supported me through a seemingly never-ending educational career. 


\section{Table of Contents}

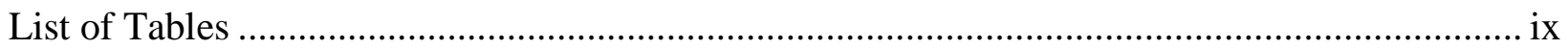

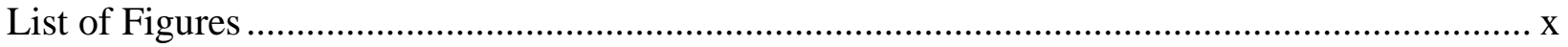

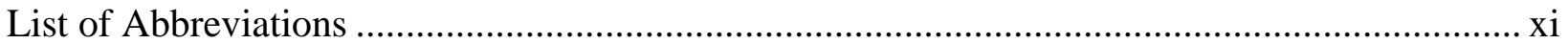

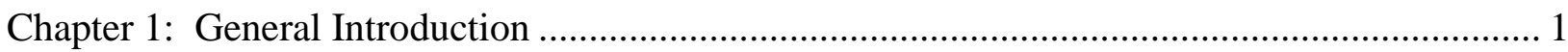

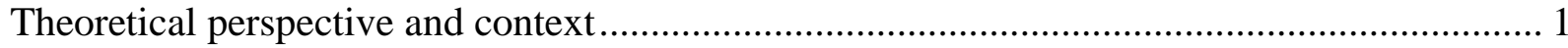

Review of rationale and brief overview of experiments .................................................... 3

Chapter 2: Literature Review for Experiment 1 ................................................................ 7

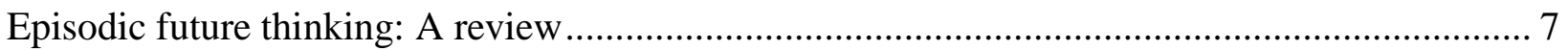

Autobiographical memory and episodic future thinking: An aging perspective .................... 11

Episodic future thinking: Beyond the brain ................................................................... 13

Autobiographical memory, future thinking, and the self.............................................. 15

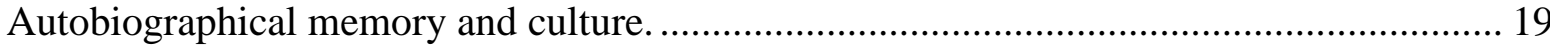

Autobiographical memory, episodic future thinking, and the social context.................... 20

Changes in personal goals and age differences in future thinking. ................................. 22

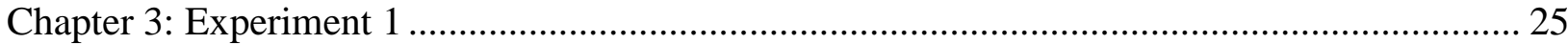

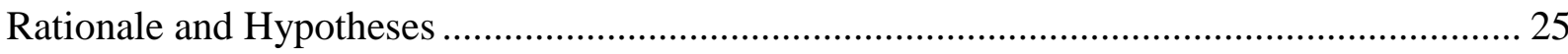

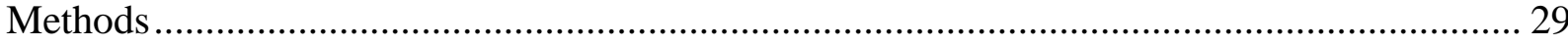

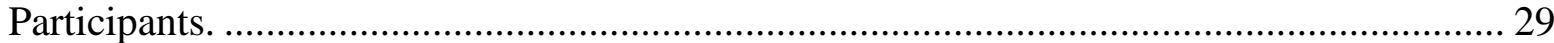

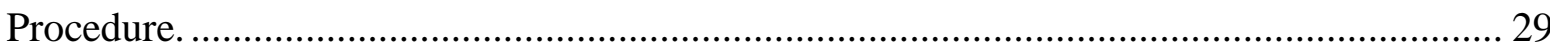

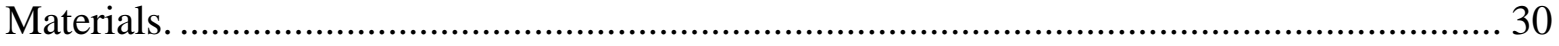

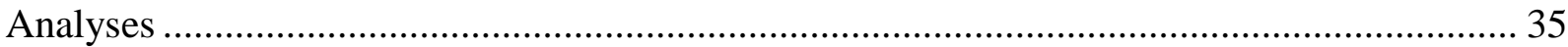

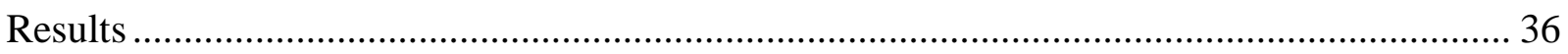

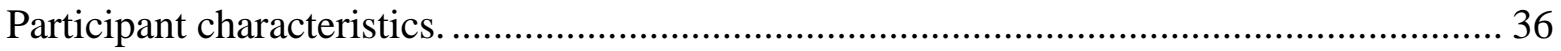

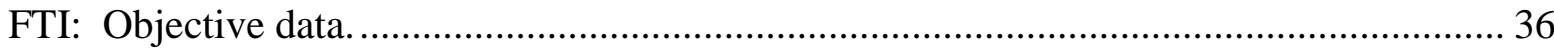

Phenomenological ratings: Subjective data........................................................... 37

Additional analyses: Practice (Non-goal) Trials. ............................................................ 39

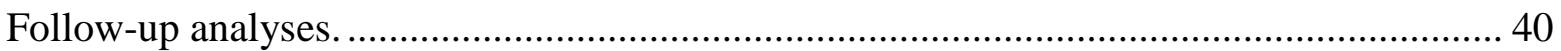

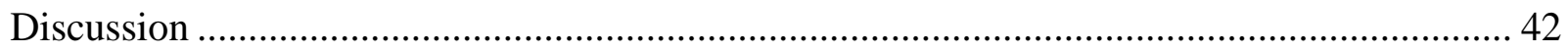

Chapter 4: Literature Review for Experiment 2 ........................................................... 51

Prospective memory, future thinking, and goal achievement ............................................. 52 


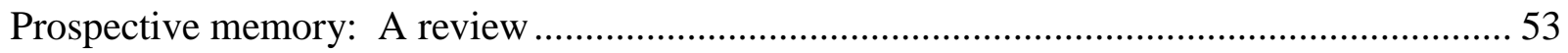

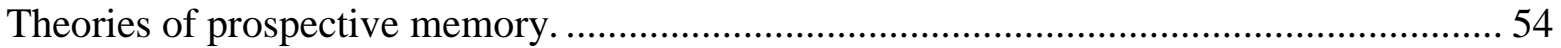

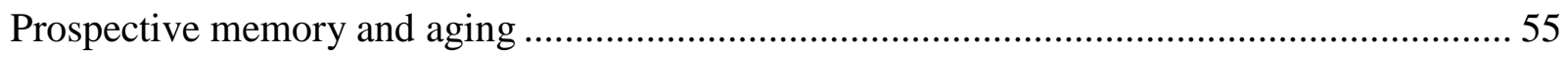

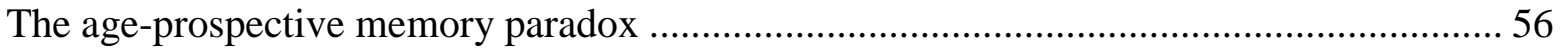

Evidence for episodic future thinking as a mnemonic aid for prospective memory:

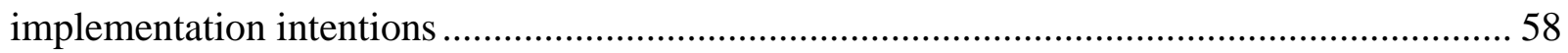

Evidence for episodic future thinking and prospective memory improvement ..................... 60

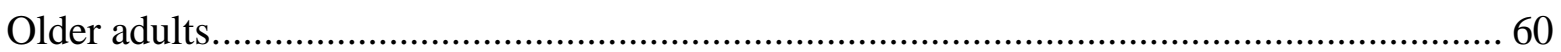

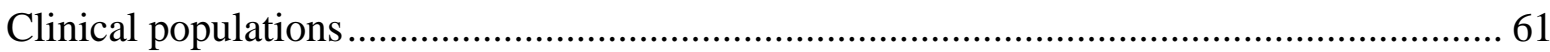

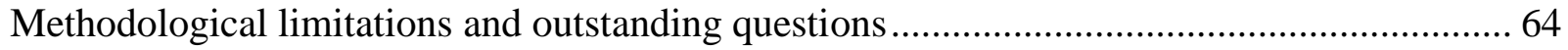

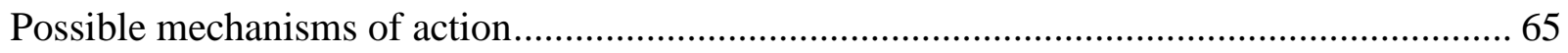

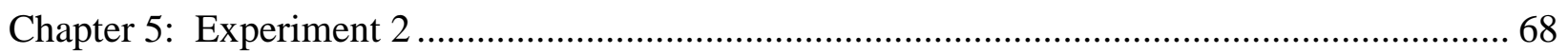

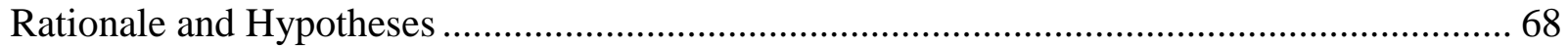

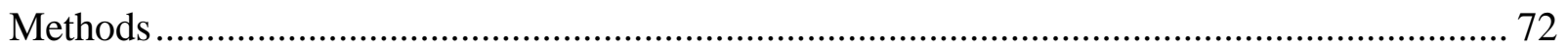

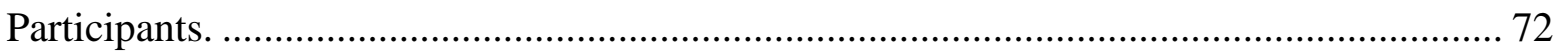

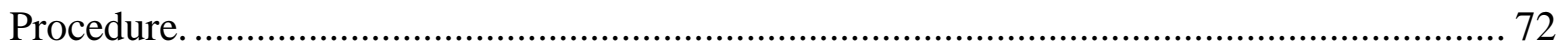

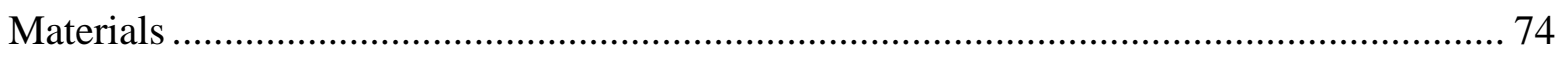

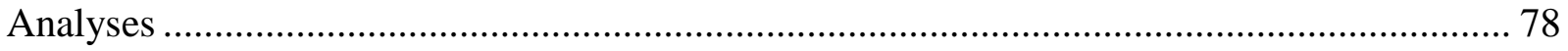

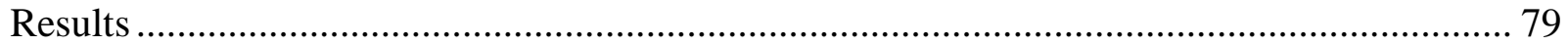

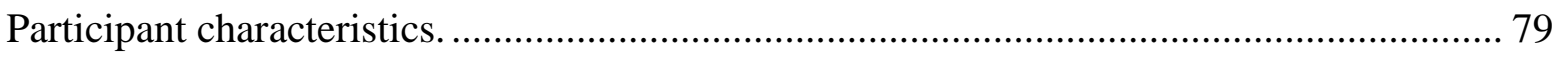

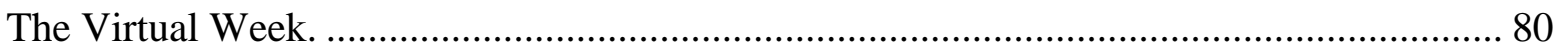

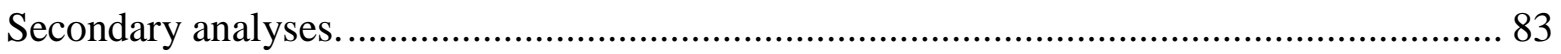

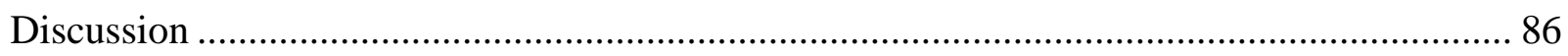

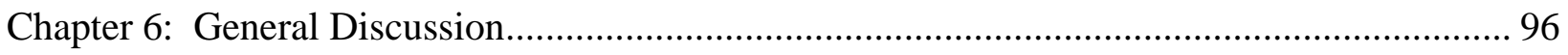

Summary and Implications of Experiment 1................................................................ 99

Summary and Implications of Experiment 2 ........................................................ 102

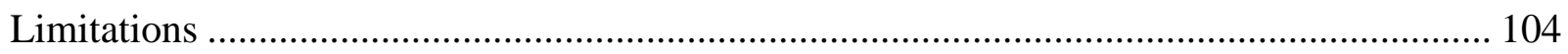

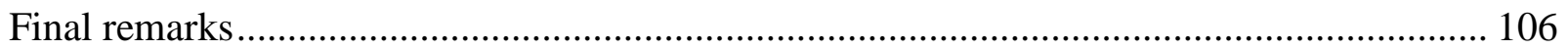

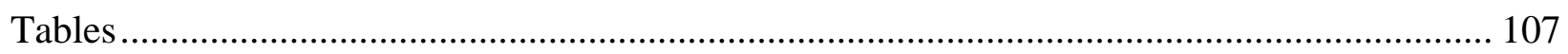

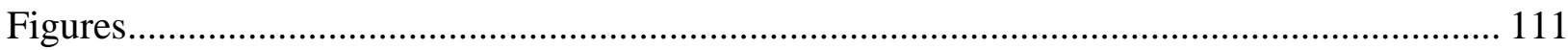

Appendix A: Follow-Up Questionnaire - Experiment 1 ............................................... 122 
Appendix B: Sample Narratives - Experiment 1 ......................................................... 124

Appendix C: Consent \& Debriefing Forms - Experiment 1 ............................................. 126

Appendix D: Consent \& Debriefing Forms - Experiment 2 ........................................... 133

Appendix E: Strategy Instruction Slides - Experiment 2 ............................................ 141

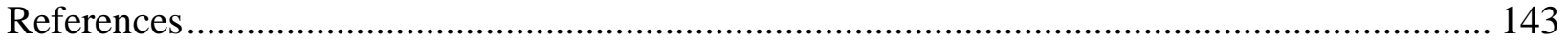




\section{List of Tables}

Table 1: Demographic and Neuropsychological Data (Experiment 1)................................ 107

Table 2: Demographic and Neuropsychological Data (Experiment 2) ................................. 108

Table 3: Virtual Week Performance ................................................................................ 109

Table 4: Distribution of Strategy Preference between Age Groups ........................................ 110 


\section{List of Figures}

Figure 1. FTI Scoring: Proportion of Internal Details................................................ 111

Figure 2. Phenomenological Ratings: Emotional Valence ............................................... 112

Figure 3. Phenomenological Ratings: Emotional Intensity ............................................ 113

Figure 4. Phenomenological Ratings: Personal Significance .............................................. 114

Figure 5. Phenomenological Ratings: Subjective Distance............................................. 115

Figure 6. Snapshot of the Virtual Week Board Game..................................................... 116

Figure 7. Depiction of Tasks within a "day” of the Virtual Week ....................................... 117

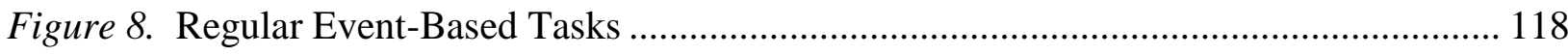

Figure 9. Regular Time-Based Tasks ............................................................................. 119

Figure 10. Irregular Event-Based Tasks .................................................................... 120

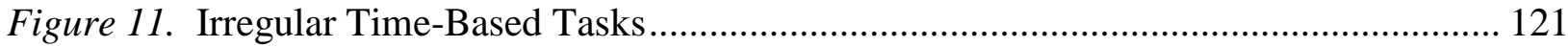




\section{List of Abbreviations}

\begin{tabular}{|l|l|}
\hline ANCOVA & Analysis of covariance \\
\hline ANOVA & Analysis of variance \\
\hline BL & Baseline \\
\hline CBT & Cognitive Behavioural Therapy \\
\hline DASS-21 & Depression Anxiety and Stress Scale 21 item version \\
\hline DS & Digit Span \\
\hline DSC & Digit-Symbol Coding \\
\hline fMRI & Functional Magnetic Resonance Imaging \\
\hline FTI & Future Thinking Interview \\
\hline FT & Future Thinking strategy \\
\hline MMSE & Mini-Mental Status Examination \\
\hline MTL & Medial temporal lobe \\
\hline PAM & Preparatory attentional and memory processes \\
\hline PANAS & Positive and Negative Affect Schedule \\
\hline PFC & Prefrontal Cortex \\
\hline PM & Prospective memory \\
\hline RIS & Remembering-imagining system \\
\hline SAM & Survey of Autobiographical Memory \\
\hline SMS & Self-memory system \\
\hline SOC & Selectivity, optimization and compensation \\
\hline SPSS & Statistical Package for the Social Sciences \\
\hline TMT & Trail Making Test \\
\hline VPA & Verbal Paired Associates \\
\hline VVIQ & Vividness of Visual Imagery Questionnaire \\
\hline VW & Virtual Week \\
\hline WAIS-IV & Wechsler Adult Intelligence Scale $4^{\text {th }}$ Edition \\
\hline WMS & Wechsler Memory Scale \\
\hline WT & When-Then strategy \\
\hline
\end{tabular}




\section{Chapter 1: General Introduction}

\section{Theoretical perspective and context}

Within the field of cognitive aging, it is well-established that healthy aging is associated with declines in episodic memory, attention, processing speed, and executive functions (Grady, 2012 for a review). Consequently, there has been a focus on understanding the functional and structural neural underpinnings of these changes. Depending on the task, older adults may show less activation in relevant brain areas as compared with younger adults, which is taken to reflect cognitive deficits (Grady, 2012). In other tasks, older adults show more activation, which is often interpreted as compensation, lack of efficiency, or dedifferentiation (Grady, 2012). Structurally, aging has been associated with volumetric declines in specific brain areas, such as the prefrontal cortices, and to lesser degrees, the temporal, occipital, and parietal cortices (e.g., Raz \& Rodrigue, 2006). These structural and functional changes are reflected behaviourally, and prominent theories at this level of explanation assert that aging is associated with declines in inhibition (Zacks \& Hasher, 1994), decreases in cognitive resources (Craik, 1986), and difficulties processing contextual information (Braver et al., 2001). It is proposed that these neural and cognitive changes underpin the declines in memory, attention, processing speed, and executive function typically observed in older adults.

However, research from lifespan perspectives has emphasized factors beyond brain-based

changes, focusing on the relationship between motivation and cognition (e.g., Hess, 2014). From this perspective, it is argued that due to changes in circumstances and experiences that occur with aging, there are increases in the selectivity and engagement of cognitive resources, which are understood as adaptive, and can help explain some of the typical age-related changes in cognition (Hess, 2006). 
One of these changing circumstances includes the increased cognitive costs to cognitive activities that come with aging (Hess, 2014). Because of this, older adults become more selective in how much they invest in cognitive activities and which ones they choose to pursue. In other words, motivation is a moderator of age differences (Hess, 2014). This selectivity is not only a function cognitive cost, but also due to changes in developmental goals (Hess, 2014). Indeed, many theories of lifespan development theorize about why and how goals change with age. The selectivity, optimization and compensation (SOC) model, which is a more general lifespan theory, proposes that because of age-related changes in cognitive resources, individuals must become increasingly selective and direct their efforts away from resource and skill acquisition towards maintenance and loss prevention of current resources (Baltes, 1997; Freund \& Ebner, 2005). Socioemotional selectivity theory posits that because of an increasingly limited time perspective, older adults come to prioritize more emotional and meaningful goals, as opposed to acquisition goals (Carstensen, Isaacowitz \& Charles, 1999). This theory has been called upon to explain the "positivity effect," which is found in many cognitive aging studies. For instance, older adults sometimes demonstrate better memory for positive stimuli compared with negative stimuli (Carstensen \& Mikels, 2005). Mikels, Larkin, Reuter-Lorenz and Carstensen (2005) found that once stimuli were switched from neutral to emotional images, age differences in working memory were eliminated, suggesting that age-related changes in motivation can shed new light onto how age-related changes in cognition are interpreted. The wealth of such studies underscores the fact that cognition is not strictly "cold" information processing. Rather, processes such as memory and attention have self-regulatory functions, often utilized in the service of maintaining emotional well-being (Carstensen \& Mikels, 2005). 
Emotion regulatory goals influence these cognitive processes, and because goals change with age, they must be considered when understanding cognitive aging.

\section{Review of rationale and brief overview of experiments}

In my Master's thesis, I studied how a particular type of goal construct known as “possible selves" changes with age (Lapp \& Spaniol, 2016). Possible selves reflect personal, future goal states, a type of future-oriented cognition presumed to guide behaviour and shape overall well-being (Higgins, 1997; Markus \& Nurius, 1986). Results from the thesis revealed that while particular themes of possible selves remained salient across the lifespan, new themes emerged with age. Still other types of possible selves, which had been of primary importance for younger adults, receded with age. Broadly, the dissertation extends this focus on goals and future-oriented cognition through the study of episodic future thinking and of prospective memory.

Experts are increasingly interested in delineating future-oriented cognition, otherwise known as prospection. Recently, a taxonomy of prospection was proposed (Szpunar et al., 2014), not dissimilar to taxonomies of memory (e.g., Squire, 2004), to distinguish among different facets of prospection. Szpunar and colleagues (2014) outlined four types of prospection, all of which fall along a spectrum spanning from episodic knowledge on the one pole to semantic knowledge on the other. These modes of prospection include simulation (e.g., episodic future thinking), prediction (e.g., affective forecasting), intention (e.g., prospective memory), and planning. These four modes purportedly interact to support the initial stages of conceptualizing possible future events, extending to the final stages of actually executing these events (Michaelian, Klein, \& Szpunar, 2016). Research remains in the early stages of uncovering the cognitive processes, motivational influences, emotional states, and functional 
sequelae involved in each of these modes of prospection. Moreover, how and why these modes may be modulated by age is unclear. The dissertation is part of this larger effort to characterize age differences in prospection, as well as to empirically validate some of the theoretical claims about the functions of the different modes of prospection.

The first dissertation experiment adds to the literature concerning how and why episodic future thinking (simulation) changes with age (Lapp \& Spaniol, 2017). Episodic future thinking is a cognitive ability that appears to decline with age (e.g., Addis, Wong \& Schacter, 2008). Typically, these changes are presumed to reflect deterioration in episodic memory and other related domains, but evidence also suggests that motivational and social factors may contribute to some of the age differences (e.g., Trunk \& Adams, 2009). Moreover, emerging evidence suggests that like episodic memory, episodic future thinking is also organized by personal goal structures (e.g., Conway \& Pleydell-Pearce, 2000; D’Argembeau \& Mathy, 2011). Given the Master's thesis work with possible selves, described earlier, which illustrated how this particular goal construct evolves with age (Lapp \& Spaniol, 2016), the first dissertation experiment examined whether activating age-congruent goal states may facilitate future thinking. That is, the first experiment investigated the nature of episodic future thinking in older adults, and in particular, how motivational factors might influence this cognitive process.

The second experiment investigated the function of episodic future thinking with respect to goal achievement. Claims about an instrumental relationship between episodic future thinking and goal achievement have some support from the self-regulation and motivation literatures, where future thinking has been a technique of interest for decades (see Taylor, Pham, Rivkin \& Armor, 1998, for a brief review). Often called by other names such as mental simulation or 
visualization, research has demonstrated that imagining oneself carrying out future, goal-directed behaviours can have positive impacts regarding planning and actual goal execution.

Likewise, research from the cognitive training and rehabilitation literatures has suggested that imaginal and simulation techniques may improve memory and planning (for reviews see Fish, Wilson, \& Manly, 2010; Hering et al., 2013; Piras, Borella, Incoccia, \& Carlesimo, 2011). Prospective memory, which is the memory to execute some behaviour in the future after a delay, is considered an example of goal-directed behaviour. As such, it represents a prime platform from which to explore claims about the beneficial role of episodic future thinking on goal achievement. Moreover, older adults commonly report difficulties with prospective memory in everyday life (Kliegel \& Jager, 2006), providing yet another impetus to uncover methods to enhance it.

Studies from the neuroscience and cognitive aging literatures on prospection also suggest that a primary function of episodic future thinking is to facilitate planning, problem solving and other goal-directed behaviours, including prospective memory (e.g., Brewer \& Marsh, 2010; Schacter, Addis, \& Buckner, 2008; Szpunar, 2010; Terrett et al., 2016). Such studies have suggested that episodic future thinking can be utilized to enhance prospective memory performance (e.g., Altgassen et al., 2015). Other studies also suggest a relationship between episodic future thinking and prospective memory through overlapping neural correlates between both modes of prospection (e.g., Stokes, Thompson, Cusack, \& Duncan, 2009). Finally, a recent study (Terrett et al., 2016) examined performance on both episodic future thinking and prospective memory tasks in younger and older adults, finding significant, positive correlations between them in both younger $(r=.27, p<.01)$ and older adults $(r=.28, p<.01)$. For younger adults, episodic future thinking accounted for a significant amount of the unique variance in 
prospective memory performance, which was greater than any of the other predictors except for retrospective memory ${ }^{1}$. The second dissertation experiment sought to extend this line of work by testing predictions about the functional role of episodic future thinking in goal-oriented behaviour by investigating the effects of a future thinking manipulation on prospective memory in younger and older adults. This experiment informs theories regarding the function of episodic future thinking, and tests its possible utility as a mnemonic aid for prospective memory, a cognitive domain of great importance for older adults.

The dissertation is divided into the following sections. Chapter 2 provides an in depth review of the future thinking literature and its relationship to goals and motivation in an aging context. Chapter 3 outlines the details of the first dissertation experiment. Chapters 4 and 5 follow the same structure with respect to the second dissertation experiment. Chapter 6 synthesizes both experiments, contextualizing them within current literature, identifies limitations and speculates on future directions.

\footnotetext{
${ }^{1}$ Interestingly, episodic future thinking did not account for any unique variance in prospective memory for the older adults, which the authors hypothesize might reflect the group's inability to utilize episodic future thinking as a strategy.
} 


\section{Chapter 2: Literature Review for Experiment 1}

\section{Episodic future thinking: A review}

Research into episodic future thinking has exploded in the past 10 years. Episodic future thinking, which has been referred to as a type of prospection (Buckner \& Carroll, 2007) or mental simulation (Schacter \& Addis, 2007), appeared in the psychological literature for decades, but the recent growth was spurred by advances made in memory research. For instance, it was discovered that both H.M. and K.C., famous patients who suffered significant lesions to their medial temporal lobes that compromised their episodic memory for personal experiences, had also lost the ability to imagine their own personal futures (Rosenbaum et al., 2005; Tulving, 1985). Both patients, when asked to imagine what they would do tomorrow, indicated that their minds were blank or provided only unspecific, vague responses (Tulving, 1985). Tulving (1985) defined autoneotic consciousness, which is the ability to represent and become aware of subjective experiences in the past and future through 'mental time travel' (e.g., Wheeler et al., 1997). These patients had lost their autoneotic consciousness, unable to project themselves into the past or forward into the futures (Rosenbaum et al., 2005; Suddendorf \& Corballis, 1997; Tulving, 1985). Through these concepts - autonoetic consciousness and mental time travel memory and future thinking were conceptually and empirically connected.

Subsequent research corroborated the finding that deficits in autobiographical memory are associated with difficulties in future thinking, including in patients with amnesia (e.g., Gamboz et al., 2010; Kwan, Kurczek, \& Rosenbaum, 2015; Rosenbaum et al., 2005; Tulving, 1985), patients with Alzheimer's disease (Haj, Antoine, \& Kapogiannis, 2015), or patients with other neurological conditions (for a review, see Irish \& Piolino, 2015). 
Later research using neuroimaging confirmed that autobiographical memory and episodic future thinking rely on overlapping brain areas (e.g., Addis, Wong, \& Schacter, 2007; see Benoit $\&$ Schacter, 2015 for a review). Both processes engage a core network involving parts of the medial cortical surface, the hippocampus and parahippocampus, and the lateral temporal and inferior posterior parietal cortices (Benoit \& Schacter, 2015). Furthermore, both processes tap into the "default network," which comprises the medial prefrontal, temporopolar, hippocampus, parahippocampus, and parietal regions (Addis et al, 2007; Maguire, 2001; see Schacter, Chamberlain, Gaesser \& Gerlach, 2012 for a recent review).

Research from the priming and the false memory literatures exposed the fallibility of memory (e.g., Loftus, 2003; see Schacter, 2012 for a review) and confirmed early claims that episodic memory retrieval is a constructive process (Bartlett, 1932). Synthesizing these findings, Addis and Schacter (2007) articulated the constructive episodic simulation hypothesis. This hypothesis suggests that an error-prone episodic memory system makes episodic future thinking possible. A memory system that is flexible, where memories are reconstructed from various sources with each recollection, allows for the imagination of infinite hypothetical future scenarios, which is extremely adaptive.

Indeed, many researchers have speculated about the function of future thinking, mostly related to coping and goal achievement (e.g., Szpunar, 2010). As noted in Chapter 1, Szpunar and colleagues (2014) proposed a taxonomy of future thinking to include four domains: simulation, prediction, intention, and planning. Each domain shares the quality of imagining a specific, autobiographical event that could take place in the future. Episodic future scenarios have been suggested to facilitate the coordination of future behaviour (Spreng \& Levine, 2006), goal achievement (e.g., Pham \& Taylor, 1999), problem solving (Gerlach, Spreng, Gilmore \& 
Schacter, 2011; Sheldon, McAndrews, \& Moscovitch, 2011) and action planning (D'Argembeau Renaud, O., \& Van der Linden, 2011).

Despite the wealth of similarities between autobiographical memories and future thinking, studies have documented important differences as well. Narratives of future scenarios tend to be rated as less detailed and more positive than personal memories (e.g., Szpunar, 2010 for a review). The lessened detail relates to both sensory and contextual (e.g., visuospatial) features (e.g., D’Argembeau \& Van der Linden, 2006). Future thinking also produces more brain activity as compared with autobiographical memory (e.g., Addis et al., 2007), most notably in the anterior hippocampus. In the meta-analysis by Benoit and Schacter (2015), the results showed that episodic simulation engages specific nodes, such as those located in the left dorsolateral prefrontal cortex and posterior inferior parietal lobe, more strongly than seen with episodic memory. In a naturalistic, observational study of future thinking (D'Argembeau et al., 2011), future-oriented thoughts were found to be highly frequent in everyday life. Indeed, previous research has found that individuals spend almost twice as much time thinking about the future than they do about the past (Jason, Schade, Furo, Reichler \& Brickman, 1989). While a portion of this future thinking is not about specific future events (e.g., imagining a specific outing with friends next Saturday, versus musing about possible, future changes in the political leanings of a country), individuals nonetheless spend much of their time thinking conceptually about the future (D’Argembeau et al., 2011).

Within the field of philosophy, and more specifically, the philosophy of memory, the extent to which future thinking and memory are related is contested. Perrin and Michaelian (2017) describe two stances on the matter: continuists, who believe that future thinking and episodic memory are two poles of the same mental time travel spectrum, and thus represent 
variations of a fundamental cognitive ability, versus discontinuists, or those thinkers who believe that future thinking and episodic memory represent distinct abilities. They parse these two stances even further: for example, they define extreme continuists as those who maintain that the only difference between future thinking and episodic memory is the temporal perspective, whereas moderate continuists might acknowledge that differences exist (e.g., future thinking is more effortful and implicates additional brain areas) but that overall, they engage the same fundamental processes. Moderate continuists are most similar to mainstream neuroscientific and psychological perspectives, whereas philosophers have traditionally ascribed to discontinuists viewpoints (Perrin \& Michaelian, 2017).

Perrin and Michaelian (2017) describe extreme and moderate discontinuists, as well. The gist of the discontinuist stance (and indeed the crux of the debate between continuists and discontinuists) is metaphysical: there is a distinct causal link between episodic memory and past events, which is not the case for future events. As such, episodic memory and future thinking are different in kind (Perrin \& Michaelian, 2017). In addition, discontinuists draw on the neuroscientific and psychological literature that describes important differences, as reviewed earlier, such as how future thinking tends to be more positive and less detailed (e.g., Szpunar et al, 2010) and engages additional brain areas (e.g., Addis et al., 2007), which they take as evidence that episodic memory and future thinking differ in kind rather than simply degree (Perrin \& Michaelian, 2017). Perrin and Michaelian (2017) also describe how discontinuists propose that the functions of episodic memory and future thinking, respectively, differ. Whereas memory is utilize for problem solving and self-regulation, future thinking - as evidenced by its positivity bias - is used to maintain self-image, support emotion regulation, and approach new problems. As such, future thinking likely has a different role with respect to identity. Finally, 
discontinuists maintain that epistemologically, episodic memory differs from future thinking in that it is possible to misidentify features of past events when one remembers (e.g., misremembering an individual or object) due to the fixed, causal relationship with the remembered event, whereas this is impossible with future episodes since they have not occurred ("[future thinking] is immune to error through misidentification"). While the debate between continuists and discontinuists is unresolved, critical questions about the nature, function, and relationships between memory and future thinking are being discussed, which may provide insights for psychologists and neuroscientists.

\section{Autobiographical memory and episodic future thinking: An aging perspective}

The intersection between episodic future thinking and memory is also supported by findings from the cognitive aging literature. However, whereas age-related deficits in episodic memory are well-documented (Craik \& Salthouse, 2000), fewer studies have investigated agerelated changes in the ability to imagine the future (for a recent review, see Schacter, Gaesser, \& Addis, 2013). Nevertheless, for both autobiographical memory and episodic future thinking, older adults tend to produce less detailed responses (fewer "internal," or episodic details) than do younger adults (Addis, Wong \& Schacter, 2008; Levine et al., 2002). At the same time, older adults add significantly more semantic and tangential details to their responses. Abram and colleagues (2014) assessed five different age groups for both autobiographical memories and future thinking abilities across a temporal continuum. Their results replicated the finding that older adults produce fewer episodic details and more semantic details for both memories and future scenarios compared with younger adults. Further, subjective ratings indicated that older adults reported less sense of re-experiencing (or pre-experiencing) their events, which is 
consistent with a shift towards semantic retrieval. In other words, older adults describe a reduced autonoetic experience in both temporal directions.

Brain changes have also been detected between younger and older adults when they imagine their future (Addis, Roberts \& Schacter, 2011). These age differences have been contextualized within the larger body of cognitive aging literature, which suggests that generally, there are age-related deficits in episodic memory (e.g., Zacks et al., 2000) whereas semantic memory is preserved or even improved (e.g., Adams et al., 1997). More specifically, aging has been associated with a general reduction in episodic specificity, related to declines in prefrontal cortex integrity (Cabeza, Anderson, House, Mangels, \& Nyberg, 2000). Levine et al. (2002) suggested that these brain changes may cause older adults to lose "resolution power" such that they remain at higher, more general levels of autobiographical memory during the retrieval process. As such, rather than eventually retrieving specific, episodic details, they retrieve disproportionately more semantic and nonspecific information (Craik \& Grady, 2002), and this appears to extend to episodic future thinking.

The reconstructive nature of both autobiographical memory retrieval and episodic future thinking requires the integrity of relational processes and importantly, older adults also tend to show difficulties with binding and relational abilities (e.g., Naveh-Benjamin, 2000). Relational memory, just like autobiographical memory and episodic future thinking, engages the hippocampus (Giovanello, Schnyer, \& Verfaellie, 2004). In support of the constructive episodic simulation hypothesis, it has been shown that for both younger and older adults: (i) level of episodic details generated for autobiographical memories correlates with the number of details for future events, and that (ii), degree of impairment on measures of relational memory correlates with degree of episodic detail for past and future events (Addis, Wong \& Schacter, 2007; but see 
Cole, Morrison, \& Conway, 2013, and D'Argembeau et al., 2010, for a lack of relationship between behavioural measures of relational memory and episodic future thinking). Both age groups demonstrate left hippocampal engagement when retrieving past events, and additionally recruit the right hippocampus for future events, which is interpreted to reflect the increased relational processing demands (Addis et al., 2007). Based on the constructive episodic simulation hypothesis, the age differences in internal details for both past and future events are attributed to changes in relational and episodic memory (Addis et al., 2008).

At the same time, evidence suggests that the age differences in these processes are not exclusively related to episodic processes. Studies have shown that generating autobiographical memories as well as future scenarios also depends on other cognitive domains such as executive function (D'Argembeau, Ortoleva, Jumentier \& van der Linden, 2010) and working memory (e.g., Abram et al., 2014; Cole et al., 2013). Both of these domains also show declines with age and have been shown to predict some of the decreases in specificity in both autobiographical memories and future scenarios (Abram et al., 2014). Semantic memory and conceptual knowledge have also been found to play key roles in episodic future thinking (e.g., Irish, Addis, Hodges, \& Piguet, 2012; Irish \& Piolino, 2015). But beyond other cognitive domains, there is theory and research to suggest that motivational factors might also influence the age differences in episodic future thinking (e.g., Trunk \& Adams, 2009).

\section{Episodic future thinking: Beyond the brain}

In addition to the evidence suggesting that the difficulties older adults have with episodic future thinking stem from cognitive declines in domains such as episodic memory, research from social psychology indicates that broader contextual factors also play a role. As alluded to in the introduction, memory, like future thinking, is not only explained by the nuts and bolts of 
information processing, storage and retrieval. Contextual factors such as motivation and social context, as well as the characteristics of the rememberer and their audience significantly shape autobiographical memory (Bluck \& Alea, 2011). As a specific example, Qi Wang, a leading researcher of culture and autobiographical memory, describes how European Americans tend to recall more positive, specific, one-time episodes that depict a unique aspect of their experience, whereas their Asian counterparts tend to recall more neutral, general, repeated events that often focus on social interactions (Wang, 2016). Wang (2016) also reported that European Americans appear to have greater access to a larger number of memories, including early events. She stressed the need to conceptualize autobiographical memory within a culturally dynamic system, which shapes personal goals as well as social norms. Social norms, in turn, influence how memory is used, starting in early childhood. Moreover, she suggested that future thinking should also be understood within a similar culturally dynamic context. Indeed, emerging empirical research is suggesting that these and additional contextual factors are also integral to understanding episodic future thinking.

While theories from cognitive psychology and neuroscience propose that a primary function of autobiographical memory is to allow episodic future thinking, research from social psychology contains theories about the functions of autobiographical memory that extend to the construction of identity, relationships, and goal achievement. For example, in a well-known model, Alea and Bluck (2003) proposed three distinct functions of autobiographical memory: self-continuity, forging social bonds, and directing future behaviour. The latter, directive function relates explicitly to future thinking, and is similar to the conceptualizations within cognitive neuroscience described earlier. More specifically, the directive function contends that autobiographical memory is specifically used to guide future thought and behaviour (Alea \& 
Bluck, 2003) through the imagination of future scenarios. Given the overlap between this directive function and earlier sections, it will not be reviewed again. However, the first two functions add to the earlier sections by elaborating how motivational features affect memory and future thinking. According to the model, the first two functions of autobiographical memory the self and social - directly modulate autobiographical memory itself, and given the strong connection between autobiographical memory and episodic future thinking, some of these same components might be important to consider with respect to the latter. As such, the self and social functions of autobiographical memory will be briefly reviewed below and extended to how they might relate to future thinking as well.

Autobiographical memory, future thinking, and the self. While the emphasis has been on uncovering the cognitive and neural underpinnings of mental time travel, several findings have also highlighted the relationship between autobiographical thinking and the self. Indeed, one of the hallmarks of autobiographical thinking is that it involves the self (e.g., Chessell, Rathbone, Souchay, Charlesworth, \& Moulin, 2014; Conway, 2005). Autobiographical thinking is involved in how individuals maintain a coherent sense of self across time, often through the formation of an autobiographical narrative (McAdams, 2006). This narrative is extended in time, encompassing both semantic and episodic features of one's past as well as hopes and goals for the future (Rathbone, Conway \& Moulin, 2011). In fact, individuals with impaired episodic memory, such as those with Alzheimer's disease, have been shown to experience a decrease in the sense of a "temporally extended self" (Addis \& Tippett, 2004) and a decreased sense of self-concept (Martinelli, Anssens, Sperduti \& Piolino, 2013).

The relationship between the self and autobiographical memory is formalized by Conway and Pleydell-Pearce (2000)'s self-memory system (SMS), which explains how autobiographical 
memory is constructed. Most importantly however, the model explains how autobiographical memories support, maintain, and are shaped by personal goals, which are critical for a sense of a coherent self. The model therefore predicts that memories related to current goals should be most accessible.

According to Conway and Pleydell-Pearce's (2000) model, autobiographical memories are selected based on the autobiographical knowledge base, which comprises (i) the semantic knowledge about who the self was, is and can be, and (ii) the working self. Autobiographical memories are retrieved in a hierarchical fashion, moving from the general, more semantic levels towards specific, episodic events. More specifically, the autobiographical knowledge base organizes information into life periods, which concern general themes in one's life, followed by general events, which are more specific representations of general or repeated events, and finally, into event-specific knowledge, which are actual episodic events. The working self consists of the conceptual self and of the individual's goal system (Conway \& Loveday, 2015). Memories are activated based on selections made by the working self. Moreover, the working self filters what experiences become stored in the autobiographical knowledge base (Conway \& Loveday, 2015). Thus, the activation of specific autobiographical memories results when, in response to a cue, the working self activates particular representations across the hierarchy of the autobiographical knowledge base (Conway \& Loveday, 2015). Placing the findings reviewed earlier into this model, older adults appear to remain at conceptual and semantic levels of retrieval and do not use the episodic memory system (i.e., event-specific knowledge) as much as is characteristic of younger adults.

Many empirical studies support the strong association between goals and autobiographical memories. McAdams et al. (1997) found that individuals who strongly 
endorsed the Eriksonian notion of generativity tended to produce autobiographical events that were indicative of this goal. Holmes and Conway (1999) demonstrated that when older individuals were required to recollect memories from each decade of their lives, they tended to produce memories that were characteristic of typical psychosocial goals of that decade or life period. Conway and Holmes (2004) had older adults freely recall memories from multiple life stages. Thematic analysis of memories for various psychosocial themes indicated that memories that were once highly relevant to the individual during a particular life period (e.g., identity formation during young adulthood) remain highly accessible when memories from that particular period are recollected. Thus, one of the important functions of the working self is to maintain accessibility of memories that are related to salient goals (Conway, 2005). Taken together, individuals appear to hold "self-defining" memories, which are an accessible set of memories that support the sense of self (e.g., Lardi, D'Argembeau, Chanal, Ghisletta, \& Van der Linden, 2010).

Recent evidence is suggesting that likewise, episodic future thinking is also organized hierarchically, and also strongly shaped by personal goals. For instance, D’Argembeau and Mathy (2011) demonstrated that the construction of episodic future episodes generally begins with the establishment of general knowledge of one's personal future, which provides the framework into which episodic details may be added later. They also demonstrated that this general personal knowledge is organized according to personal goals: cueing participants with personally relevant goals facilitated the formation of future events (see also Christian et al., 2013; Demblon \& D’Argembeau, 2014; Stawarczyk \& D’Argembeau, 2015). Cole and Berntsen (2015) measured voluntary and involuntary mental time travel, or the ability to mentally relive past events or preview future ones (Wheeler et al., 1997). They found that future-directed mental 
time travel was more strongly related to current concerns and goals than were memories. Furthermore, representations related to current concerns and goals were rated as more vividly imagined, supporting the assertion that autobiographical thought connected to salient goals is more accessible.

More recently, it has been shown that individuals also support their identity through the imagination of meaningful goals and events (e.g., D’Argembeau, Lardi \& Van der Linden, 2012). Moreover, studies have shown that anticipated future events may even be rated as more important than memories in defining the self (Addis, Wong \& Schacter, 2008; Berntsen \& Bohn, 2010). Of course, the notion that individuals hold future representations of the self is not new (D’Argembeau et al., 2012). For example, Markus and Nurius (1986) proposed the concept of possible selves, encompassing both who one aspires to be and who they are afraid of becoming. Any given individual can hold a multitude of possible selves, which emerge and fade in significance over the lifespan (e.g., Frazier, Cotrell \& Hooker, 2003; Smith \& Freund, 2002). With age, individuals tend to hold fewer possible selves across fewer life domains, suggesting a narrowing, selecting and focusing of future goals (Cross \& Markus, 1991). While possible selves do not necessarily require an episodic component, research has shown that individuals do indeed hold a set of significant future events that are easily accessible, frequently thought about, and define important features of the self (D'Argembeau et al., 2012). The way in which individuals construct self-defining narratives of autobiographical events correlates with the ways in which they also construct such future events (D'Argembeau et al., 2012). Understood within the SMS (Conway \& Pleydell-Pearce, 2000), possible selves can be understood to reflect the conceptual and general levels of the autobiographical knowledge base, while the construction of specific future events reflects the subsequent engagement of the episodic system (D'Argembeau 
et al., 2012). Indeed, D’Argembeau and Mathy's (2011) findings provide preliminary evidence for such an organization of the future thinking process.

Other studies have supported this relationship between memory, future thinking, and the self. Pillemer and colleagues (2013) captured memories and episodic future events in younger adults. They found that achievement themes in both memories and future events, which are particularly salient for younger adults, were associated with higher ratings for positive selfregard, as compared with other themes.

Autobiographical memory and culture. Memories and future thinking are also shaped by larger, culturally shared goals and semantic knowledge. The 'cultural life script' is composed of culturally specific events that are expected to occur throughout a prototypical lifespan (Berntsen \& Rubin 2004). Presumably, it also guides the construction of both autobiographical memories and episodic future thinking (Bohn \& Berntsen, 2013; Grysman, Prabhakar, Anglin, \& Hudson, 2010). Berntsen and Jacobsen (2008) studied the nature of involuntary memories and future events and found that life script events are more likely to be generated when future events were imagined farther into the future (rather than closer to the present), suggesting that perhaps individuals require such normative, conceptual scaffolds to imagine an increasingly uncertain future. Berntsen and Bohn (2010) asked participants for memories and future events in response to cue words as well as in response to requests for important events. They found that when asked for important past and future events, participants tended to describe events that were part of the cultural life script, whereas life script events were significantly less frequent in response to random cue words. Further, as events increased in temporal distance from the present, they tended to rely more on life script events, suggesting that the life script is particularly helpful for mental time travel to farther life periods both past and future (Berntsen \& Bohn, 2010). 
Furthermore, Grysman, Prabhakar, Anglin, and Hudson (2014) found that while both memories and episodic future thinking rely on the cultural life script, the latter depends on it more strongly, perhaps because of the greater uncertainty and difficulty in generating such events.

Berntsen and Bohn (2010) also found that importance for the self (i.e., "self-narrative") increased with temporal distance, which was interpreted within temporal construal theory (Trope \& Liberman, 2003). According to this theory, temporally distant events consist of higher level, more conceptual representations, whereas events that are closer to the present involve more specific, contextual and concrete details (Berntsen \& Bohn, 2010). This provides additional evidence that both autobiographical memory and episodic future thinking are governed by similar underlying systems. Overall, the evidence suggests that both memories and imagined scenarios strongly relate to the sense of self, and that the activation of semantic knowledge, in the form of "cultural life scripts" or in the form of personal goals, may facilitate mental time travel.

\section{Autobiographical memory, episodic future thinking, and the social context. The} second function of autobiographical memory identified by Alea and Bluck (2003) is social. Indeed, many researchers have claimed it is the most fundamental function of autobiographical memory (e.g., Neisser, 1988). Sharing autobiographical memories with others provides material for conversation, which might be in the service of communicating or teaching information, social bonding, or increasing intimacy (e.g., Pasupathi, Lucas \& Coombs, 2002).

It has been suggested that older adults may hold different communication goals than younger adults, which may be reflected in differences in narrative style and content. Trunk and Adams (2009) assessed communication goals in younger and older adults when delivering either episodic or procedural narratives. While younger adults preferred expressive goals for episodic 
narratives and objective goals for procedural narratives, older adults did not exhibit such consistent goals. Rather, older adults tended to report a mix of both goals for both types of narratives. It was concluded that younger adults may produce less tangential speech - which would be reflected as higher internal details in autobiographical and future thinking paradigms as a function of specific communication goals, which are not necessarily shared with older adults.

Although not measured with respect to personal past and future events, research has investigated how the relationship between the rememberer and listener can influence narratives. Adams and colleagues (2002) studied how younger and older adults recounted a story they learned to either the experimenter or a young child. Results indicated that both younger and older adults adapted their retellings when the young child was the listener, by including more elaborations and repetitions. Age differences occurred only when the experimenter was the listener. Overall, the older adults were found to adapt their retellings to their listeners more than did younger adults, suggesting that the communication goals and narrative style were particularly important for the older adults and help to explain some of the age differences noted. Both studies suggest that the social context can significantly influence narratives, and that older adults may be differentially impacted by such contextual factors.

Changes in narrative style have also been attributed to other sources. It has been suggested that older adults have acquired more general knowledge and wisdom (Labouvie-Vief \& Blanchard-Fields, 1982) and prioritize providing context (Levine et al., 2002), which might translate into greater semantic and elaborative detail when recalling past or imagining future scenarios. Gaesser and colleagues (2011) compared younger and older adults for past and future thinking but also for a picture description task in order to tease apart narrative or descriptive 
styles from episodic processes. They found that older adults showed similar deficits in internal details across all three tasks, and because the picture task does not rely on episodic processes, the authors concluded that mechanisms other than episodic processes must be considered; in fact, performance on the picture task explained most of the variance in performance on the past and future thinking tasks.

Changes in personal goals and age differences in future thinking. As regards the self function of autobiographical memory, there are significant age-related changes to goals and identity that might be of importance. Described earlier, cultural life scripts provide a heuristic for autobiographical memory retrieval and future event generation. Importantly, life scripts differ between younger and older adults (Rubin, Berntsen \& Hutson, 2009). For younger adults, the script includes marriage, having children, landing a first job, settling on a career, and the death of a loved one. For older adults, the script includes parents' death, empty nest, grandchildren, retirement, and one's own death. The life script represents normative goals and expectations, and which change with age and likely influence the construction of past and future events in different ways. Grysman and colleagues (2014) studied the relationship between the cultural life script and future thinking in younger and older adults. Replicating previous findings, they showed that life script events were more frequent when events were imagined farther into the future, and there was a positivity bias in both age groups. Despite the fact that the life scripts of older adults tend to include more negative events as compared with younger adults, the positivity effect was observed across the lifespan, regardless of whether a life script event was generated or not. Still, whereas younger adults generated events about their own life script, older adults more frequently imagined attending life script events of others. Thus, despite 
some similarities across the lifespan (i.e., the maintenance of the positivity effect), the specific future events varied as a function of age-related changes in the life script.

On an individual (rather than cultural) level, goal orientation also changes with age. Recent work in our laboratory has addressed the idea of lifespan changes in goal orientation by analyzing the content of self-reported goals ("possible selves") in younger and older adults (Lapp \& Spaniol, 2016). Adapting a method used in prior studies on possible selves (Markus \& Nurius, 1986), research participants were asked to list attributes that described the person they "hoped to be" or the person they "ought to be." Presumably, possible selves help regulate current behaviour by providing a point of reference (Higgins, 1983). Individuals are motivated to decrease the discrepancy between hoped-for selves and to avoid becoming feared future selves in order to maintain a sense of well-being (Carver \& Scheier, 1982). We investigated how discrepancies between current and future selves change with age, and also performed thematic analyses of the specific types of selves generated by the younger and older participants. Our results indicated that the magnitude of discrepancy between current and future selves does not change with age but that the types of selves do. Both younger and older adults prioritized interpersonal goals, naming possible selves such as "caring" or "loving" most frequently. However, there were also important shifts that occurred with age. Older adults generated significantly more possible selves from the domain of duties and obligations, characterized by attributes such as "punctual" and "responsible," as well as from the domain of health, including attributes such as "fit" and "healthy." In contrast, younger adults generated significantly more possible selves characteristic of achievement themes, such as "successful" or "leader." Thus, both older and younger adults were able to project themselves into the future in terms of an abstract, semantic self-construct, and the types of future selves they envisioned showed both 
stability as well as change. These findings are consistent with motivational theories of lifespan development (e.g., Baltes, 1997; Carstensen, Isaacowitz, \& Charles, 1999; Ebner , Freund, \& Baltes, 2006), which postulate age-related shifts from the pursuit of gains and the acquisition of resources, knowledge and relationships, towards the prevention of losses and the maintenance of resources, relationships, and positive experiences. Thus, the changing goals of younger and older adults are expressed in abstract, conceptual future projections, and it may be possible that there is also a relationship between motivational themes and episodic future thinking. 


\section{Chapter 3: Experiment 1}

In the previous chapter, the directive relationship between autobiographical memory and episodic future thinking was reviewed, which provided a context for understanding the agerelated changes documented in episodic future thinking. There is compelling evidence from cognitive psychology and neuroscience suggesting that these age-related changes in episodic future thinking are underpinned by the same brain-based changes observed in autobiographical memory, such as decrements in relational binding. However, research was also reviewed from social psychology, including cross-cultural studies and lifespan theories of development, that support a significant role of motivation and other contextual factors in shaping autobiographical memory and episodic future thinking. A prominent theory of autobiographical memory (Conway \& Pleydell-Pearce, 2000) acknowledges the primary role that personal goals play in the selection and formation of autobiographical memories, and there is evidence to suggest this same system shapes episodic future thinking. The first dissertation experiment is nested within this theoretical work and aims to test hypotheses about how age-related changes in personal goals might relate to variance in episodic future thinking patterns observed across the lifespan. ${ }^{2}$

\section{Rationale and Hypotheses}

More specifically, the present research investigates how personal goals, which are agedependent, may modulate the ease with which individuals produce simulations of future scenarios. To our knowledge, the contribution of goals in explaining age differences in episodic future thinking has not been explored. Adapting the typical cueing paradigm utilized to measure episodic future thinking, participants were prompted with words relating to themes that differ in motivational salience between younger and older adults. Broadly, we expected that activating

\footnotetext{
${ }^{2}$ The results of this experiment have been published in Memory (Lapp \& Spaniol, 2017). The methods, results, tables, and figures are all presented in this dissertation as they appear in the peer-reviewed, published manuscript.
} 
motivational themes that are more or less important to younger and older adults should modulate the degree of episodic detail produced. Older adults should benefit from the activation of personal goals. The self-reference effect, which describes how memory can be significantly boosted when the information to be remembered is encoded with reference to the self (e.g., Hamami, Serbun \& Gutchess, 2011) is maintained with age (Gutchess, Kensinger, Yoon, \& Schacter, 2007), suggesting that older adults remain sensitive to self-related information. In a population that shows pronounced difficulties with episodic thinking, Kwan and colleagues (2015) found that cueing episodic future thinking in individuals with MTL amnesia using more detailed, personally meaningful sentences (instead of single, decontextualized cue words) significantly enhanced episodic detail in three of the five patients. The authors suggest that, depending on the extent of neurological and/or cognitive damage, episodic future thinking can be facilitated when personally meaningful goal structures are activated. This may provide a type of semantic scaffolding onto which individuals can then add episodic richness (Kwan et al., 2015). Indeed, this is consistent with the semantic scaffolding hypothesis proposed by Irish and colleagues (2012; Irish \& Piguet, 2013), as well as the view that episodic future thinking, like memory, should be considered within the context of motivated cognition.

Regarding specific hypotheses, we predicted that older adults would generate fewer episodic details and more semantic details than younger adults overall (Addis et al., 2008; Gaesser et al., 2011; Levine et al., 2002). Critically, however, we predicted that these age differences would depend on goal domain, based on previous findings of age differences in goal salience $^{3}$ (Lapp \& Spaniol, 2016). In a domain that is more salient for younger adults than for older adults (achievement), we expected that age differences in episodic future thinking would be

\footnotetext{
${ }^{3}$ For a reminder, this was reviewed in detail at the end of Chapter 2.
} 
particularly large. In a domain that is equally important to younger and older adults (interpersonal relationships), we expected the age differences would be of intermediate size. Finally, in domains that are more salient for older adults than for younger adults (duties/obligations, and health), we expected that the age differences would be minimized.

In addition to quantifying episodic and semantic details using a standardized measure, participants provided subjective, phenomenological ratings of their future simulations: vividness, emotional valence and arousal, personal significance, and imagined date of the event (see Addis et al., 2008). Our hypotheses regarding these phenomenological characteristics were more tentative, since prior studies that have captured self-reported phenomenological ratings have been mixed (e.g., Addis et al., 2008; Addis, Musicaro, Pan, \& Schacter, 2010). We expected that perceived vividness of the imagined scenarios should show a Cue X Age interaction, with those cues most salient for younger or older adults, respectively, being associated with greater ratings of vividness. This would be consistent with some previous research indicating that mental time travel is perceived as more vivid the more closely it relates to current concerns and goals (Cole \& Berntsen, 2016; Singer \& Blagov, 2004).

Concerning valence, hypotheses were also speculative. On the one hand, there is reason to predict that older adults would rate their responses more positively than younger adults: previous studies support an age-related positivity effect in autobiographical memory (e.g., Carstensen \& Mikels, 2005; Singer, Rexhaj, \& Baddeley, 2007). On the other hand, Chessell et al. (2014) did not find an age difference in emotional valence between younger and older adults when they imagined future scenarios; however, their paradigm for eliciting episodic future thinking differed from ours. As such, the question of age differences and emotional valence remains unanswered. We also predicted that older adults would rate their future simulations as 
more emotionally arousing than younger adults, which has been found previously (e.g., Addis et al. 2008; Addis et al., 2010; Addis, Roberts, \& Schacter, 2011). It was also expected that cues more salient for younger or older adults, respectively, should elicit more positive and arousing ratings, although the evidence for this is less strong. Cole and Berntsen (2016) found that mental time travel related to goals was rated more positively and intensely than representations that were unrelated, but this study did not compare younger and older adults.

Again, age differences in personal significance ratings in episodic future thinking studies have been conflicting (Addis et al., 2008; 2010); thus, it is difficult to make clear hypotheses. However, we did expect a Cue X Age interaction based on the fact that the cues should represent themes varying in salience, depending on age. Thus, we expected that younger adults would rate responses to achievement cues as most salient and older adults would rate responses to health and duties/obligation cues as most salient. We expected no age differences for responses to the interpersonal cues since this theme was reported equally often by younger and older adults in the original study (Lapp \& Spaniol, 2016).

Finally, we expected that younger and older adults would rate their imagined events as occurring relatively close to the present day; that is, most responses should be expected to occur in the next few months rather than the next few years. Prior work has shown that individuals tend not to cast their events very far into the future (if the date is unconstrained) and that this pattern holds across younger, middle-aged and older adults (Spreng \& Levine, 2006). Presumably, it is cognitively demanding to project events farther into the future as there is less certainty and as a result, more novelty to construct. Regarding group differences, Chessell et al. (2014) found that older adults imagined their future events closer to the present as compared with younger adults, perhaps because of a more limited future time perspective. Socioemotional 
selectivity theory (Carstensen, Isaacowitz, \& Charles, 1999) posits that the priority of personal goals is influenced by perceptions of remaining time left to live; older adults have been consistently found to have a more limited future time perspective, presumably due to the relationship between chronological age and time left to live (e.g., Lang \& Carstensen, 2002).

\section{Methods}

Participants. Thirty-four older adults ( 30 females; mean age $=72.06$ years; range 65 85 ) and 33 younger adults ( 28 female; mean age $=20.91$; range $18-30$ ) completed the study at Ryerson University. This sample size is based on our previous work with younger and older adults and provides sufficient statistical power $(\geq .90)$ to detect medium-sized between- and within- group main effects and interactions ${ }^{4}$. Numbers of males and females did not differ significantly between age groups. Older adults were recruited from an existing database of selfselected older adults interested in research participation and were compensated $\$ 24$ for their participation. Younger adults were recruited from introductory psychology classes in the Ryerson psychology department and received partial course credit for their participation. Exclusion criteria - assessed via interview with an experimenter - for both age groups included: current or past (within 3 years) psychiatric diagnosis, psychiatric medication, learning disability, neurological disease, uncontrolled thyroid or heart conditions, cancer (past 3 years), and any history of symptomatic concussion or head injury. In addition, older adults were excluded if they scored below 26 on the Mini-Mental Status Examination (MMSE; Folstein, Folstein, \& McHugh, 1975).

Procedure. Ethical approval was received from the Ryerson University Ethics Board. Participants were tested individually by the first author or one of two trained research assistants.

\footnotetext{
${ }^{4}$ Established using $G^{*}$ power 3.1 with alpha $=.05$.
} 
Upon providing written consent, participants completed paper and pencil baseline measures (described below), followed by the adapted Future Thinking Interview (FTI; Addis et al., 2008). Once finished, they repeated another baseline measure, the Positive and Negative Affect Schedule (PANAS; Watson, Clark, \& Tellegen, 1998) for a second time, as well as a demographics screening interview (younger adults only; older adults were screened over the phone prior to participation), and finally older adults completed the MMSE. All participants were then debriefed and compensated for their participation. Sessions took approximately 1 hour for younger adults and 1.25 hours for older adults.

\section{Materials.}

Baseline measures. Participants completed Digit Symbol Coding, a measure of processing speed (WAIS-IV, Wechsler, 2008), the Shipley Vocabulary test (Shipley, 1940), which is a multiple-choice measure of crystallized intelligence, and two self-report scales: the Depression Anxiety and Stress Scale 21 (DASS-21; Lovibond \& Lovibond, 1995) and the PANAS (Watson et al., 1998). The DASS-21 comprises 21 items that measure depression, stress, and anxiety. The PANAS contains two subscales of 20 items that assess state affect. The MMSE (Folstein et al., 1975) is a commonly used instrument to screen for cognitive impairment.

Adaptive Future Thinking Interview. The FTI (Addis et al., 2008) is a future-directed version of the past-directed Autobiographical Interview (Levine et al., 2002). In response to cue words, participants were asked to imagine and describe aloud plausible but novel future scenarios that could possibly happen to them. In keeping with the original version, participants were also instructed to generate scenarios that are specific in time and place, lasting no longer than a few hours or one day at maximum. It was emphasized that they should make the scenarios as rich as possible and may use their five senses to aid them in producing details. They 
were encouraged to include details about what they imagined seeing, hearing, tasting, smelling or touching in their simulations. They were asked to imagine their future scenarios from a field perspective (rather than observer perspective). Finally, participants were told that they had a maximum of three minutes to talk but that they could take however much time was necessary to think of a scenario to describe (instructions based on similar studies such as Addis et al., 2008). The administrator only began timing once the participant began to speak. If responses were shorter than 2 minutes and 20 seconds, the administrator asked once if the participant had any further details to add. If participants reached the time limit, they were gently cut off from further responding. Participants were free to imagine their event at any time point in the future. See Appendix B for sample narratives from a younger and an older adult.

Instructions and cue words were presented on the computer screen using E-prime 2.0 (Psychology Software Tools, Inc.). Participants advanced through the instruction slides at their own pace. Participants' understanding was queried by the administrator following each instruction slide to ensure full understanding. Participants then completed two practice trials and received feedback from the administrator. Each participant saw the same cue words, in the same order, for the practice trials. The first practice trial word was "dress," which is typical of cue words used in other episodic future thinking studies. The second practice trial word was "entertained." This word was selected because it was less concrete than the first, making it more comparable to the cue words used in the experimental trials. If a participant did not understand the instructions during the practice trials, or began to describe a memory or a very general event instead, the participant was stopped, reminded of the instructions, and asked to begin again. Timing was restarted for these select cases. Once participants demonstrated full understanding of the task, they began the experimental trials of the FTI. 
In contrast to the original version of the FTI, where the cue words are neutral and unrelated to personal goals, the cue words were selected for this task to represent the four goal domains that emerged from a previous study about changes in personal goals across the lifespan (Lapp \& Spaniol, 2016). These domains include: interpersonal relationships, achievement, duties and obligations, and health. In the adapted FTI, 2 cue words were chosen per goal domain, yielding a total of 8 cue words 5 . All participants saw the same cue words, and cue order was counterbalanced across participants. In order to be consistent with the original version, participants were told that their responses did not have to involve the cue word itself; rather, participants could free-associate in response to each cue word. It was explained that each cue word was simply a prompt for the imagination. One limitation to our study is that the cue words were uniquely selected for this study and as such, differed from the non-goal-related cue words that have been used in previous episodic future thinking studies. Due to concerns about participant fatigue, an additional control condition, whereby cue words were unrelated to goals, similar to previous studies, was not included. Consequently, responses to goal-related cue words cannot be directly compared to responses to non-goal-related cue words, which might cloud comparisons made with previous studies. In the attempt to address this limitation, secondary analyses (see section Additional Analyses) were conducted in which data from the 2 practice trials were compared with those from the experimental trials, since the cue words employed for the two practice trials were not related to personal goals and were the same as those used in other episodic future thinking studies (e.g., "dress"). We expected that in response to non-goal-related words, the pattern of age differences would match what previous studies have

\footnotetext{
${ }^{5}$ The 4 goal domains are as follows, with the 2 cue words listed in parentheses: achievement (successful; famous); duties and obligations (organized; punctual); physical health (healthy; active); and interpersonal conscientiousness (kind; friendly).
} 
found: Older adults should produce significantly fewer internal (episodic details relating to the central event) and more external (non episodic details, including semantic, tangential, or repeated information) details than younger adults. Moreover, we expected that the magnitude of age differences in response to non-goal-related words would be comparable to those in response to goal-related words that are equally salient for both age groups (e.g., interpersonal relationships).

For the duration of the task, the administrator sat beside the participant and discreetly monitored the time of each response. No feedback was given for the 8 trials, although participants were reminded each time to imagine specific and detailed events. If participants began to describe a memory instead or clearly forgot the instructions, they were reminded of the instructions by the administrator and timing was restarted for the new response.

Following each response, participants provided a series of numerical ratings in response to questions about phenomenological characteristics of the future scenario they had just generated (Addis et al., 2008). These included: vividness ( $1=$ vague, to $5=$ very vivid), emotional valence $(1=$ very negative, to $5=$ very positive $)$, emotional intensity $(1=$ non emotional, to $5=$ highly emotional $)$, personal significance $(1=$ insignificant, to $5=$ life changing), field or observer perspective, and the approximate date of the generated event ( $1=$ next few weeks; $2=$ next few months; $3=$ next few years).

Adapted FTI scoring. Responses were audio recorded and later transcribed for scoring according to the standardized Autobiographical Interview procedure (Levine et al., 2002). Responses were de-identified and then assigned by an external assistant blind to the study hypotheses to an administrator that had not tested that particular participant. Although scorers were blind to group membership, at times it was possible to identify group membership based on response content. All administrators were trained as reliable scorers according to the 
Autobiographical Interview training procedures prior to the commencement of the study. Interrater reliability of scoring assessed with intraclass correlations, established on the basis of 20 events $^{6}$ scored by the administrators, was high (>.85 for internal and external detail scores).

To score a particular response, scorers first identify the central event of the narrative (that is, the event that is specific in time and place). If multiple events were generated in one response, the central event is that which is composed of the greatest number of internal details. Next, responses are segmented into discrete units, which are then classified as "internal" details (i.e., episodic information related to the main event, such as, "It's Tuesday, next week, and I imagine myself walking into my exam in the great hall") or "external" details (i.e., semantic details, repetitions, or details irrelevant to the main event, such as "It's very important to be punctual. I'm a lot more punctual than I used to be"). These coarse categories are subsequently broken down further into different types of internal details (e.g. event, time, place, emotion, etc.) and external details. For each response, the number of each detail type is tallied, and the totals are averaged across the two responses for each cue type to create internal and external scores for each cue type for each participant.

Follow-up Scoring Analyses. As stated earlier, although the goal themes were derived from prior empirical work (Lapp \& Spaniol, 2016), participants in this study were not asked to rank the cue words per se (or goal themes that the words were assumed to reflect) in order of personal importance. In response to an anonymous reviewer comment received about our manuscript during the peer-review process, we collected additional data approximately one year after the study was completed in order to assess the validity of the study stimuli. The older adults from the original study $(\mathrm{N}=32$; two participants could not be reached) were re-contacted.

\footnotetext{
${ }^{6}$ Events were from the standardized scoring set from the Levine protocol, which is the typical practice for establishing reliability amongst scorers.
} 
With their consent, we mailed them a questionnaire (see Appendix A) to determine the ranked order of importance of each goal theme. In addition, to confirm that the cue words did in fact reflect the goal theme (i.e., interpersonal, achievement, duties/obligations, or physical health) they were chosen to represent, the questionnaire asked participants to rate which goal theme best reflected each cue word. Because the younger adults in the study were drawn from first year psychology classes, it was not possible to re-contact these same individuals due to stipulations of the University ethics board. As such, a new sample of younger adults $(\mathrm{N}=32)$ completed the questionnaire via online survey. Independent $t$ tests showed that the new sample did not differ from the original sample of younger adults in terms of demographic characteristics, with one exception: the original sample reported significantly more stress than the new sample, $t(62)=$ $2.44, p<.05$. Otherwise, the samples did not significantly differ on the depression or anxiety subscales of the DASS, or baseline levels of affect as measured by the PANAS.

\section{Analyses}

Demographics and baseline measures were analyzed using $t$-tests and chi-square tests. To investigate experimental hypotheses, the proportion of internal details relative to overall number of details was submitted to mixed analyses of variance (ANOVAs) with 2-level between-subjects (age: younger and older) and 4-level within-subjects factors (goal domain: interpersonal, achievement, duties/obligations, physical health). Likewise, the results from the phenomenological ratings were submitted to similar ANOVAs ${ }^{7}$. Exploratory correlational analyses were also performed to investigate relationships between the baseline measures, the

\footnotetext{
${ }^{7}$ While the ratings might be classified as ordinal data, parametric tests were utilized as these tests are robust and appropriate for the mixed design of the experiment. Parametric tests can be a valid option for these data (Velleman \& Wilkinson, 1993) given the experimental questions and hypotheses, and there is precedent within the autobiographical memory and episodic future thinking literature for analyzing similar data in this fashion (Viard et al., 2011).
} 
future thinking interview scoring, and the phenomenological ratings made by participants.

Because the positive PANAS scores correlated with some of the outcome measures, additional

analyses (analyses of covariance; ANCOVA) were run using group mean centered values for this variable (Schneider, Avivi-Reich, \& Mozuraitis, 2015). The results of these additional analyses are reported only if they diverged from the original results.

Violations of sphericity were corrected using the Greenhouse Geisser correction. Significant results from the omnibus tests were followed up using Bonferroni corrections. The alpha level was set to .05 , and all tests were two-tailed.

\section{Results}

Participant characteristics. Demographic characteristics are displayed in Table 1. Older adults had significantly more years of education and demonstrated higher levels of crystallized intelligence but had significantly slower processing speed. Younger adults reported significantly higher levels of anxiety and stress according to the Depression, Anxiety and Stress Scales (DASS-21; Lovibond \& Lovibond, 1995) scores, but the groups did not differ in terms of depressive symptoms. These measures did not correlate significantly with any of the main outcome measures after controlling for age and thus were not included in the main analyses.

Regarding state affect, older adults reported significantly higher levels of positive affect both before and after the main task. Meanwhile, levels of negative affect did not significantly differ between groups. Change scores were analyzed separately within each age group using paired $t$-tests, which indicated that neither group reported significant changes in positive or negative affect across the session.

FTI: Objective data. Younger and older adults did not differ significantly in terms of total number of details produced in their responses, and this effect held across all cue types, 
indicating that the total verbiage produced was not significantly affected by goal domain (see

Figure 1). They nevertheless evinced differences when types of details were compared. The Detail (2 levels: internal/external) X Cue (4 levels) X Age (2 levels: younger and older) ANOVA revealed a main effect of Detail, $F(1,65)=33.21, p<.001, \eta_{p}{ }^{2}=.34$, but no main effects of Age, $F(1,65)=.43, p=.52, \eta_{p}{ }^{2}<.01$, or goal domain (Cue), $F(3,195)=.51, p=.68$, $\eta_{p}{ }^{2}<.01$. There was also a significant Detail X Age interaction, $F(1,65)=46.80, p<.001, \eta_{p}{ }^{2}=$ .42. Follow-up tests showed that, in keeping with previous findings (e.g., Addis et al., 2008), younger adults produced significantly more internal details, $t(65)=4.13, p<.001, d=1.01$, while older adults produced significantly more external details, $t(65)=-6.70, p<.001, d=1.64$. To corroborate this pattern, the proportion of internal details relative to total details was also submitted to a mixed ANOVA, which showed a significant effect of Age, $F(1,65)=70.93, p<$ $.001, \eta_{p}{ }^{2}=.52$. Neither the main effect of cue type, $F(3,195)=.61, p=.61, \eta_{p}{ }^{2}<.01$, nor the interaction, $F(3,195)=94, p=.43, \eta_{p}{ }^{2}=.01$, was significant.

\section{Phenomenological ratings: Subjective data.}

Vividness. Mixed ANOVAs indicated that older and younger adults did not significantly differ in terms of self-reported vividness of their responses. There was also no effect of cue type, nor a significant Cue X Age interaction.

Emotional valence $^{8}$. The mixed ANOVA revealed a main effect of cue type, $F(3,195)=$ $15.48, p<.001, \eta_{p}{ }^{2}=.19$, driven by the fact that overall, responses to the duties and obligation cues were rated as significantly more negative than the rest, which showed no differences from

\footnotetext{
${ }^{8}$ Because the positive PANAS scores (pre-FTI) correlated with the emotional valence ratings and because these scores significantly differed between younger and older adults, an ANCOVA was run using them as a covariate. The pattern of results changed only slightly: the main effect of age became significant, $F(1,64)=4.01, p=.049, \eta_{p}{ }^{2}$ $=.06$, whereas before it was only a trend. The other effects were unchanged.
} 
each other (see Figure 2). There was a trend for a main effect of group, $F(1,65)=3.74, p=$ $.057, \eta_{p}{ }^{2}=.054$, whereby overall, older adults tended to make more positive ratings than younger adults. There was also a significant Cue $\mathrm{X}$ Age interaction, $F(3,195)=5.10, p<.01$, $\eta_{p}^{2}=.07$. Within the younger adults specifically, they made significantly higher ratings in response to the achievement cues as compared with duties/obligations cues $(p<.001)$ and physical cues $(p<.01)$ while there was no difference with responses to interpersonal cues. Further, younger adults' responses to the duties/obligation cues were significantly more negative than all other cue types ( $p<.001$ for interpersonal and achievement; $p<.05$ for physical).

On the other hand, for older adults, responses to the physical cues were significantly more positive than responses to duties and obligations $(p<.01)$, but responses to this latter cue did not differ from the other two cue types (i.e., interpersonal and achievement). Thus, for older adults there was less variation in the ratings, and achievement was not as salient as it was for the younger adults. Younger adults viewed achievement responses as particularly positive (i.e., highest ratings) and duties/obligation cues as particularly negative (i.e., lowest ratings).

Emotional intensity. There was a significant main effect of cue type, $F(3,195)=12.29$, $p<.001, \eta_{p}{ }^{2}=.16$, as well as a significant Cue X Age interaction, $F(3,195)=7.97, p<.001, \eta_{p}{ }^{2}$ $=.11($ see Figure 3). There was no main effect of age. Bonferroni-corrected post hoc tests indicated that achievement was significantly more arousing than all other cue types $(p<.01$ for interpersonal; $p<.001$ for duties and obligations and for physical). The interaction was driven by the younger adults, who rated responses to the achievement cues as more arousing than all other cue types ( $p<.001$ for all three), while for older adults, there were no significant differences between cue types. In other words, responses to the achievement cues were more arousing than responses to all other cue types, but only for the younger adults. 
Personal significance. In contrast to Addis et al. (2008), who reported no age group difference in personal significance ratings (albeit for neutral cues), we found a significant effect of age, $F(1,65)=9.29, p<.01, \eta_{p}{ }^{2}=.13$, indicating that older adults made significantly higher ratings overall than the younger adults (see Figure 4). There was a main effect of cue as well, $F(2.58,167.35)=8.34, p<.001, \eta_{p}{ }^{2}=.11$, with responses to achievement cues rated as more personally significant than all others $(p<.001$ for interpersonal and physical cues; $p<.05$ for duties and obligations). Finally, there was a significant Cue X Age interaction, $F(2.58,167.35)$ $=11.95, p<.001, \eta_{p}{ }^{2}=.16$. Younger adults rated responses to achievement cues as more personally significant than all other cue types $(p<.001)$, while older adults rated responses to all cue types as equally significant.

Subjective distance. Participants were asked to indicate when in the future they imagined their event to occur (i.e., next few weeks, next few months, or next few years). There was a main effect of Cue, $F(3,195)=16.03, p<.001, \eta_{p}{ }^{2}=.20$, where responses to achievement cues were projected farther into the future than all other cues types $(p<.001)$. There was no main effect of Age and no significant Cue X Age interaction (see Figure 5).

Perspective. No results were significant here, indicating that all participants followed the instruction to imagine their simulations from the field perspective rather than from an observer perspective, and that this was not modulated by cue type.

\section{Additional analyses: Practice (Non-goal) Trials.}

To explore potential differences between non-goal-related and goal-related words, a mixed Cue (5 levels: practice, interpersonal, achievement, duties/obligations, physical health) by Age (2 levels: younger and older) ANOVA was run. There was a main effect of Cue, $F(4,244)$ $=4.08, p=.003, \eta_{p}^{2}=.06$, and of Age, $F(1,61)=73.23, p<.001, \eta_{p}{ }^{2}=.55$. Consistent with 
prior studies (e.g., Addis et al., 2008) and with the results from the experimental trials exclusively, older adults produced a lower proportion of internal details as compared with younger adults. Pairwise comparisons indicated that a greater proportion of internal details were produced in the practice trials compared with each of the experimental trials except for the physical health domain. There was no significant Cue X Age interaction, however, $F(4,244)=$ $1.70, p=.15, \eta_{p}{ }^{2}=.03$, suggesting that the cue effect was similar for both age groups ${ }^{9}$.

The phenomenological ratings from the practice trials were compared with the experimental trials to investigate if goal-related words elicited a different pattern of ratings compared with non-goal-related words. Mixed Cue (5 levels: practice, interpersonal, achievement, duties/obligations, physical health) by Age (2 levels: younger and older) ANOVAs were re-run separately for each of the phenomenological ratings. The pattern of results was unchanged when compared to the main analyses; that is, the pattern of ratings did not differ between practice and experimental trials. All main effects and interactions remained significant, and no new effects emerged.

\section{Follow-up analyses.}

Word ratings. Following the FTI, participants were asked to rate each of the 8 cue words for the characteristics of imageability, valence, and arousal. Correlations were run across age groups between these word characteristic ratings and the particular outcome variables where relationships might be expected (e.g. word imageability might be related to perceived vividness ratings). Results indicated that imageability word ratings for any of the cue types did not

\footnotetext{
${ }^{9}$ An additional $\mathrm{t}$ test was run comparing the proportion of internal details produced in the practice trials between age groups. This additional test was run to ensure that possible differences for the non-goal-related cues were not "washed out" by including four goal-related cues that already did not show any interaction. The test confirmed that for the non-goal-related cues, as with the goal-related cues, older adults generated relatively fewer internal details as compared with younger adults, $t(61)=5.57, p<.001, d=1.40$.
} 
correlate with the proportion of internal details derived from the FTI. Similarly, imageability word ratings did not correlate with phenomenological ratings for vividness except for the interpersonal cue type $(r=.27, p<.05)$. Valence word ratings did not correlate with the emotional valence ratings, and arousal word ratings did not correlate with the emotional intensity ratings. Given the lack of meaningful relationships between the word characteristics and outcome variables, they were not included as covariates in any of the main analyses.

Correspondence of responses to cue words. To be consistent with previous studies, participants had been instructed that the cue word was simply a prompt for their imagined future scenarios and as such, they could free-associate if necessary. Therefore it was possible that participants did not generate responses that actually involved the goal domain reflected by the cue word. To test this possibility, 15 responses were randomly selected from each age group and an independent rater, blind to the actual cue words and the overall experimental questions, matched each response to a likely cue word. The rater correctly identified 26 of the 30 responses. The rater also provided 'second guesses' for a subset of responses since some were ambiguous (e.g., responses to 'active' versus 'healthy' were sometimes difficult to differentiate). When the rater's second guesses were considered, the rater correctly identified 29 of the 30 responses. These results suggest that participants were generally providing responses reflective of the goal domains represented by the cue words.

Follow-up questionnaire. We used the follow-up questionnaire data to determine the modal ranks of each of the four goal themes. For example, the modal rank of the 'Physical Health' theme in the older-adult group corresponded to the most frequent rank assigned to this theme by older participants. Supporting the study hypothesis, the modal rankings differed across age groups. Whereas older adults ranked the goal themes in the following order of importance: 
'Physical Health', ‘Considerate' (i.e., Interpersonal conscientiousness), 'Responsible' (i.e., Duties and Obligations), and finally, 'Achievement', a less distinctive pattern emerged for the younger adults. Younger adults ranked 'Achievement' and 'Physical Health' as equally mostimportant (tied for top modal rank). This was followed by the goal theme 'Responsible,' and lastly, 'Considerate,' which was ranked as least important. Overall, the different cue words, which reflect four distinct goal themes, appear to change in importance with age.

The follow-up questionnaire also allowed us to test whether the cue words actually reflected the intended goal themes. Indeed, this was the case; both age groups provided the same pattern of rankings for each of the cue words, which validated the selection of study stimuli. For example, the cue word 'successful' was rated as most reflective of 'Achievement', and 'punctual' was rated as most reflective of the theme 'Duties and Obligations'.

Finally, correlations were run, for the older adult group only ${ }^{10}$, to explore whether individual goal theme rankings related to the objective (i.e., the FTI data) or subjective (i.e., the phenomenological ratings) data from the experimental trials. No significant correlations emerged, underscoring the lack of relationship between goals and episodic future thinking, as was suggested by the main analyses.

\section{Discussion}

The results from this study indicate that the episodic future thinking deficits found in healthy older adults are not mitigated by the activation of age-relevant goals. Prompting episodic future thinking with age-relevant goal themes did not affect age differences in level of episodic detail, suggesting that at least with our cue word method, the age differences in episodic

\footnotetext{
${ }^{10}$ As a reminder, the younger adults who completed the questionnaire were a different sample than those who participated in the original study whereas the older adults were the same because it was possible to re-contact them. As such, correlations were only run within the older adults.
} 
future thinking are robust. Older adults preferentially provided semantic and external information in their simulations, which is in line with the constructive episodic simulation hypothesis (Schacter \& Addis, 2007). Similarly, subjective ratings of vividness were unchanged by the cueing scheme in both age groups. Thus, the episodic richness of future thinking does not appear to be enhanced by the activation of age-related goals and this null effect was observed for both age groups.

Kwan et al. (2015) distinguish between two possible mechanisms underlying the increased external details and decreased internal details found in the responses of older adults. The difficulty generating internal details might relate to deficits in relational binding (e.g., Mullally, Hassabis, \& Maguire, 2012), which is largely supported by the hippocampus. Meanwhile, the inflated external details might relate to executive dysfunction, such as problems with inhibitory control or attention (e.g., Zacks \& Hasher, 1994). Like autobiographical memory construction, episodic future thinking is thought to be a generative process in which different types of knowledge are bound together, from the general to the specific, to form a cohesive episode (Conway \& Pleydell-Pearce, 2000). This type of controlled processing is thought to involve the frontal/executive system as well as working memory (e.g., Piolino et al., 2009). Above and beyond these cognitive mechanisms however, cohort differences in narrative style (e.g., Gaesser et al., 2011) may also contribute to the particular ratio of internal to external details demonstrated by older adults, although its contribution has not been fully addressed (but see Trunk \& Abrams, 2009). Our results suggest that these mechanisms are not influenced by the activation of goals, at least with the methodology employed here.

Several possibilities may explain the null effect of goal activation on episodic future thinking in both younger and older adults. The goal domains were selected based on group 
patterns that emerged in a previous study (Lapp \& Spaniol, 2016), and the samples may have differed between studies. Although this concern was partially addressed by the follow-up questionnaire, the sample of younger adults differed from the original sample. Furthermore, although the results from the follow-up questionnaire support the premise that goal themes change in importance with age, these themes were imposed by the experimenters. Studies that allow for individualized selection of goals represent future avenues of exploration. Alternatively, it is conceivable that cueing with single words or with conceptual rather than concrete words is not sufficiently potent to activate goal representations. Future work should begin to compare different types of methods for eliciting autobiographical thinking to better understand the impact of these factors.

Lending support to this future direction, Sheldon and Chu (2016) measured differences in fluency and episodic detail when autobiographical memories were cued with theme words (e.g., accomplishments; romantic experiences) versus more concrete, spatial words (e.g., concert venue; waiting room). The results revealed that spatially cued memories were retrieved more quickly and with more episodic detail, whereas thematically cued memories were retrieved in greater quantities. This was taken as confirmatory of models like that of Conway and PleydellPearce (2000) whereby a large quantity of memories is nested within thematic or goal structures. The thematic cues were presumed to function as a top-down, organizational retrieval mechanism. The authors noted that they did not compare responses between the different thematic cues, but they hypothesized that particular themes (e.g., accomplishments) would elicit more self-defining memories across the lifespan whereas other themes (e.g., holidays) would prompt memories more central to one's personal life narrative and thus might be more constrained to a particular life period (Sheldon \& Cue, 2016). Regarding spatial cues, the authors suggested that these cues 
prompt retrieval in a more bottom-up fashion, and as such, provide efficient access to sensoryperception and emotional details (Sheldon \& Chu, 2016). They speculate that these two different types of retrieval reflect different neural networks, and may exist to serve different functions. For instance, thematic cues bring many different episodes to mind, which could be more helpful for long-term planning or for drawing conclusions based on a variety of experiences, whereas spatial cues could be more useful when automatic retrieval is demanded, like when quick solutions or decisions are necessary (Sheldon \& Chu, 2016). Overall, the results hint at the importance of types of cues utilized for prompting autobiographical thinking.

Finally, another consideration is the type of words that were selected to represent the goal domains. These words, while matched to each other for important linguistic characteristics, differed from words used in other studies that do not investigate goal-related stimuli. For instance, the degree of concreteness is different, as are other grammatical characteristics such as lexical class (i.e., verb or noun). One hypothesis is that non goal-related words, which tend to be more concrete, would elicit higher episodic detail since these types of cue words might be less abstract and thus easier to imagine. Although not one of the aims of this study, future research could focus on more fine-grained analyses of how these lexical properties may contribute to episodic future thinking in general and to age differences in particular.

On the other hand, while grammatical properties of the cue words were not examined, participants were asked to rate the cue words for imageability, valence, and arousal. The results showed that there was no relationship between these word ratings and the phenomenological ratings of future thinking responses for vividness, emotional valence, and intensity, respectively. It is unclear why the word characteristics did not meaningfully relate to the phenomenological ratings, as might have been expected. The lack of relationship suggests that the overall 
phenomenological pattern of future thinking responses found in this study is relatively robust, regardless of certain word features. At the same time, participants' phenomenological ratings may not necessarily be accurate reflections of their responses: for instance, older and younger adults did not differ in their vividness ratings and yet on the objective measure of episodic, internal details, there were significant group differences. A possible follow-up study might engage trained and blind raters to rate responses on valence and intensity and explore correlations between the word ratings and phenomenological ratings. This might reveal any relationships that might be beyond participants' perception, or it might corroborate the lack of influence these word features have on the phenomenology of future thinking, thus supporting the pattern of results found in this study.

As noted earlier, it is noteworthy that there were no age differences in self-reported vividness ratings despite the significant differences in the objective indices of internal detail. Other studies have also found a discrepancy between objective and subjective ratings. For instance, Irish and colleagues (2012) found that individuals with semantic dementia rated their autobiographical memories as equally vivid as their future simulations, despite the significant impairment in episodic future thinking uncovered by the objective index. This raises the question: What are individuals evaluating when rating perceived vividness? The authors proposed that these individuals were recasting their entire memories into the future, corresponding to similar perceptions of familiarity between conditions and hence, similar subjective ratings for vividness and emotionality. Because we did not include an autobiographical memory condition, we cannot fully corroborate this hypothesis, but it could possibly explain our own similar pattern of results. Another possibility is that because the total number of details (total verbal output) did not differ between younger and older adults, the 
participants may have been rating total verbiage provided in their responses, rather than episodic vividness per se. This underscores the importance of incorporating both objective and subjective measures of episodic future thinking, especially in the context of aging research.

While no discernible differences in levels of internal and external details, as captured by the objective scoring scheme, or self-reported vividness, were elicited by the different cue types, this was not the case for the majority of the subjective ratings. Particular phenomenological features were modulated by the activation of different goal domains, with interesting differences depending on age group. Cueing younger adults with achievement-related words corresponded to responses rated as more positive, more arousing, and as more personally significant than responses to many of the other cue types. Younger adults also showed some decreased ratings for emotional valence regarding responses to duties/obligations cues. In other words, many phenomenological features were sensitive to goal domain in the younger adults. Moreover, the fact that achievement-related cues corresponded to increased perceptions of emotionality and personal significance is consistent with lifespan theories of development. According to socioemotional selectivity theory, when time is perceived as open-ended, as it generally is for younger adults, goals tend to concern novelty and acquisition of resources, including achievement (Charles \& Carstensen, 2010). Thus, it is not surprising that responses to achievement cues were more salient for younger adults.

By contrast, older adults' subjective ratings were, on the whole, less sensitive to goal domain as compared with those of younger adults. Cue type did not affect older adults' ratings of emotional intensity or personal significance. Although there was some elevation in emotional valence for responses to the physical/health cue types, a significant difference emerged only with respect to responses to one other cue type (duties/obligations). At the same time, older adults 
were not completely resistant to the effects of goal activation. Like younger adults, they projected their responses to achievement cues significantly farther into the future than other cue types. This suggests that the older adults were able to differentiate between cue types, even though most phenomenological ratings were unaffected by cue. Overall, the older adults appear to show a dedifferentiation across the different cue types, which is not the case with the younger adults.

Collapsing across cue types, we found additional age differences in phenomenological features. Compared with younger adults, older adults rated their responses as more positive and more personally significant. This is consistent with general findings supporting a positivity effect in the cognitive aging literature (see Mather \& Carstensen, 2005, for a review). Whether older adults are actually simulating more positive scenarios or are appraising them more positively after the fact is unclear since there was no objective index of emotional valence. However, autobiographical memory research has found that older adults' memories become more positive with passing time, whereas younger adults' memories become more negative (Kennedy, Mather, \& Carstensen, 2004). This suggests that older adults tend to appraise their future simulations in a positive light, perhaps motivated by their general prioritization of emotional well-being.

For other aspects of the phenomenological experience however, younger and older adults showed similar patterns. Just as there was a lack of age difference in subjective ratings of vividness, there was no difference in emotional intensity, despite the fact that the simulations produced by older adults lacked internal details. The fact that the age groups did not differ in self-perceived emotional intensity suggests that level of internal details is not clearly related to 
emotional intensity. In other words, these results suggest that one's sense of emotional intensity is not entirely dependent on degree of re-experiencing.

There was also no age difference regarding when in the future the simulations were predicted to occur. On average, both age groups dated their events as happening within the next few months. This finding is interesting given differences in future time perspective between younger and older adults (e.g., Lang \& Carstensen, 2002). On the one hand, it is consistent with prior research that suggests it is increasingly demanding to generate future scenarios that extend farther into the future (Spreng \& Levine, 2006); most future simulations are imagined close to the present. On the other hand, it is interesting that older adults did not cast their projections significantly closer to the present compared with younger adults, given the reduced cognitive resources available for older adults.

In summary, the results from our study support the finding that age-related deficits in episodic future thinking are pronounced (Abram et al., 2014; Schacter et al., 2013), even when age-relevant goals are activated. However, phenomenological features of the future simulations are modulated by these age-relevant goals, although younger adults appear to be more sensitive than older adults. While the cue word technique is the most commonly employed methodology in future thinking research (Spreng \& Levine, 2013), other paradigms may be fruitful in future studies (e.g., Kwan et al., 2015; Sheldon \& Chu, 2016). Providing richer or more personalized cues may allow for a deeper exploration of our original hypotheses (i.e., can the activation of age-relevant goals facilitate episodic future thinking?). Indeed, there is a significant body of theoretical literature (e.g., Conway \& Pleydell-Pearce, 2000), as well as empirical work (e.g., D’Argembeau \& Mathy, 2011; D’Argembeau \& van der Linden, 2004; Kwan et al., 2015), that propose a profound relationship between motivational constructs and episodic thought. Future 
research on motivational aspects of episodic future thinking in the context of aging will help shed light on the cognitive and psychosocial processes that influence this ability. 


\section{Chapter 4: Literature Review for Experiment 2}

As mentioned in an earlier chapter, one of the supposed reasons for a fallible, constructive memory system is to allow for episodic future thinking, which in turn is presumed to be adaptive. One of the purported functions of episodic future thinking is the facilitation of goal achievement, or the successful completion of some intended action in the future. Indeed, studies have shown that engaging in particular forms of future thinking can increase the probability that an intended behaviour is actually completed in the future (Chen et al., 2015; Gollwitzer \& Sheeran, 2006; Taylor et al., 1998). The following experiment investigated this claim with older adults, using prospective memory as the model for goal-directed behaviour. As will be argued below, prospective memory represents one important form of goal intention (e.g., Penningroth \& Scott, 2007), and there are both theoretical and empirical reasons for relating it to episodic future thinking (e.g., Burgess et al., 2011; Szpunar et al., 2014). Indeed, a handful of very recent empirical studies have begun to also explore this same question. For example, Altgassen et al. (2014) had older and younger adults engage in episodic future thinking prior to completing an ecologically valid laboratory task of prospective memory and found that this intervention significantly improved performance. However, such research is still emerging and further corroboration will help validate these theoretical claims about the function of episodic future thinking. Moreover, prospective memory is a domain with which older adults commonly report trouble, so exploring how episodic future thinking might alleviate such complaints would also be of benefit to this population.

First, a brief review of goals and prospective memory is provided, highlighting how it relates to cognitive aging in particular. Next, the case is made for how prospective memory and 
episodic future thinking are related, drawing largely from the self-regulation and motivation literature. Finally, the rationale for the remaining dissertation experiment is explained.

\section{Prospective memory, future thinking, and goal achievement}

Successful goal completion requires translating vague, abstract intentions into specific behaviours that one will carry out at a later point in time. This involves an interaction between cognitive and motivational processes, which Gollwitzer (1990) captured in his model of goal attainment. The model posits four separate action phases of goal pursuit: the predecisional phase, the preactional phase, the actional phase, and the postactional phase. Each phase is associated with a particular task. In the first phase, the goal must be chosen from an array of possible desires and a commitment must be made for its pursuit. In the second phase, an action plan is created, and effective plans specify which, where and when actions should be completed (Faude-Koivisto, Wuerz, \& Gollwitzer, 2009). In the actional phase, the plan is carried out, and in the postactional phase, the results are evaluated.

Goal attainment is not always so straightforward however; many goals become derailed, distracted, or even fixed in the first or second stages, even if intentions are strong (Gollwitzer \& Sheeran, 2006). Forgetting the intention or specifics of the action plan is one common reason for failing to complete goals (Gollwitzer \& Sheeran, 2006). For example, Milne and colleagues (2006) found that $70 \%$ of participants forgot to complete planned breast exams despite having formed strong intentions to do so (Gollwitzer \& Sheeran, 2006).

Forgetting may be due to lack of cognitive resources, attentional depletion or memory failures (Einstein \& McDaniel, 1996). Within the memory literature, this failure to act is known as a prospective memory failure, and as such, prospective memory can be a critical component of successful goal achievement. Thus, prospective memory represents an opportune arena for 
exploring theoretical claims about the benefits of episodic future thinking for goal achievement, and furthermore, might provide insights into how episodic future thinking could improve cognitive functioning in older adults.

\section{Prospective memory: A review}

Prospective memory is remembering to perform a particular action or intention at some future point (Brandimonte, Einstein, \& McDaniel, 1996). It is composed of two components: a retrospective component (what is the intention) and a recognition component (cue identification) (Simons, Scholvinck, Gilbert, Frith, \& Burgess, 2006). Although these components are behaviourally separable, they both rely on the anterior prefrontal cortex, which is thought to direct attention between external stimuli (cue identification) and internal representations (maintaining the intention; Simons et al., 2006).

Common lab-based tasks of prospective memory involve performing some type of ongoing task, such as categorizing pictures or words, where a baseline of reaction time and accuracy is established. Subsequently, participants are instructed to perform a second action, such as pressing a particular key, as a function of a cue that will be added to the ongoing task, such as a sound or a particular picture or word. A delay usually follows these instructions (i.e. the retention interval), where participants may complete a filler task to prevent any rehearsal of the instructions, prior to beginning the ongoing task again with the addition of the prospective memory component. The cue does not interfere with performance of the main task. As such, difficulty is enhanced because executing the intended action is self-initiated (Gonen-Yaacovi \& Burgess, 2012; Graf \& Uttl, 2001).

Cues can be of two types: event-based or time-based. In event-based prospective memory, an external cue, often in the environment, serves to prompt the execution of the 
intended action. Time-based prospective memory involves executing the action after a specific amount of time or at a particular time in the future. Time-based tasks are typically more difficult as they require the most self-initiation (e.g., Gonen-Yaacovi \& Burgess, 2012). Cues are also distinguished based on focality to the main task. If the cue must be processed in order to complete the main task, it is classified as focal, whereas if it is not central to the main task, it is non focal. Typically, time-based and non focal cues are the most difficult types of prospective memory cues.

Performance is evaluated based on successful execution of action following the cue. In addition, costs to the ongoing task are often measured to reveal mechanisms of successful prospective memory. If performance on the main task is not significantly impacted, it may be concluded that more automatic processes explained the execution of the prospective memory task in response to the cue. However, if successful prospective memory performance came with costs to the main task, it may be concluded that changes in vigilance and attention were responsible. That is, cognitive resources were allocated away from the ongoing task towards monitoring for any presence of the cue.

Theories of prospective memory. Two main theoretical models have been put forward to explain the cognitive processes underlying prospective memory. Einstein and McDaniel (1990) initially proposed the spontaneous retrieval theory to capture participants' self-reported experience of the prospective memory intention automatically "popping" into their minds (Gonen-Yaacovi \& Burgess, 2012). Later, Smith (2003) formulated the preparatory attentional and memory processes theory (PAM theory), which stated that prospective memory relies on an effortful, resource-consuming state of vigilance concerning the appearance of the cue. This theory emerged from studies where performance during an ongoing task was compared before 
and after the addition of the prospective memory instructions and showed that successful action execution was associated with decrements to the ongoing task. Based on the contradictory evidence concerning automatic retrieval and attentional monitoring, Einstein and McDaniel (2000) revised their original theory - the multiprocess model - to propose that prospective memory can be supported by both mechanisms, depending on individual differences, cue types, and other task parameters.

\section{Prospective memory and aging}

Prospective memory is a cognitive domain with which older adults frequently report difficulties (e.g., Maylor, 1990). Self-report measures indicate that older adults complain more of prospective memory problems than of retrospective difficulties (Kliegel \& Jager, 2006; Smith, Della Sala, Logie, \& Maylor, 2000). Studies suggest that prospective memory difficulties may account for over half of all memory problems and strongly relate to maintaining functional independence in everyday living (Crovitz \& Daniel, 1984; Schnitzspahn \& Kliegel, 2009; Terry, 1988). This is not surprising; older adults tend to have trouble when tasks require controlled, self-initiated processing and when environmental supports decrease (Craik, 1986). Craik (1983) predicted early on that older adults should show large declines in prospective memory performance. Compared with other types of memory, such as free recall or recognition, prospective memory is highly dependent on self-initiated activity (Craik, 1986; Gonen-Yaacovi \& Burgess, 2012).

In spite of this prediction, early studies of prospective memory suggested that it is spared by aging so long as cues are focal, because this type of retrieval was purported to rely on automatic processes (e.g., Einstein \& McDaniel, 1990). However, this claim was contested during the following years. Later meta-analyses demonstrated that in lab-based studies of both 
event- and timed-based prospective memory, older adults perform worse than younger adults (Henry, MacLeod, Phillips, \& Crawford, 2004). Still, many studies have failed to find any age differences at all (reviewed in McDaniel, Einstein, \& Rendell, 2008).

More recent meta-analyses have sought to clarify under which conditions age differences should emerge. Kliegel and colleagues (2008) found that age differences in lab-based tasks were largest when cues were non focal to the ongoing task, but some degree of age differences still emerged in response to tasks with focal cues. Uttl (2011) refined the study selection criteria of earlier meta-analyses by accounting for prevalent ceiling effects and other methodological confounds. He found that age differences are large for lab-based tasks with both focal and non focal cues. He also argued that the effect sizes are comparable to those found in recall measures of retrospective memory, thus making a strong case against any claims that prospective memory with focal cues is spared with aging. Ihle et al.'s (2013) meta-analysis of event-based prospective memory studies demonstrated that age differences were largest when the order of the ongoing and prospective memory task responses are specified, compared with when the order is unspecified. Like other meta-analyses, age differences were also larger when cues were non focal rather than focal. Thus, it appears that age differences in prospective memory are reliable but the degree depends significantly on task and study parameters.

The age-prospective memory paradox. The most notable finding with respect to age differences is that older adults reliably outperform younger adults in event- and time-based tests of naturalistic prospective memory (e.g., Henry et al., 2004). Such tasks have included logging the time at various time points throughout the week (e.g. Rendell \& Thomson, 1999), telephoning the researcher (e.g., Matsumoto, Nishimura, \& Tabuchi, 2011; Maylor, 1990), and mailing postcards back to the researcher (e.g., West, 1988). This dissociation between lab-based 
and naturalistic tasks has been termed the age paradox (Rendell \& Craik, 2000). Researchers have postulated several explanations for the paradox (reviewed in Schnitzspahn, Ihle, Henry, Rendell, \& Kliegel, 2011). Many have suggested that lab-based tasks are more cognitively demanding; they are more abstract, less contextualized, and are less amenable to help from compensatory strategies that may be available in everyday situations. Other possibilities include: older adults may be less stressed or busy in their everyday lives, making it easier to complete naturalistic tasks; older adults may be more adept at planning, more aware of their abilities, and more inclined to use strategies and memory aids outside the lab, which may give them an advantage over younger adults (but Phillips et al.'s, 2008 review provides little support for this assertion). Furthermore, naturalistic tasks may tap into different domains and may simply be less demanding than lab-based tasks of prospective memory.

Another promising explanation concerns age differences in motivation towards the prospective memory tasks. Indeed, motivation and task importance are significant factors for prospective memory performance in general (reviewed in Walters \& Meier, 2014). Researchers have used various methods to manipulate importance, such as rewards or incentives, changing task instructions to emphasize the ongoing versus the prospective memory task, or manipulating social contingencies (Walters \& Meier, 2014). Preliminary evidence suggests that in naturalistic contexts, older adults perform better than younger adults because they value the prospective memory task more. In the lab, they might inherently prioritize the ongoing task; however this emphasis is malleable such that if the prospective memory task is prioritized, performance can be significantly improved, albeit at a cost. Understood within both the multiprocess and PAM theories, if the prospective memory task is prioritized, more cognitive resources would be directed towards this task and away from the ongoing task: more important tasks would elicit 
more intense attentional monitoring. Hering et al. (2013) point out that while this interpretation supports the assertion that older adults have more limited cognitive resources, as Hess (2014) describes, older adults still have control over how they decide to allocate these resources. Overall, these results suggest that motivation contributes to prospective memory performance and furthermore, it suggests that factors other than purely "cold," cognitive processes may influence the age differences in prospective memory. As a final consideration, the paradox is particularly striking given the frequency of difficulty older adults report regarding their prospective memory performance in everyday life (e.g., Maylor, 1990). Naturalistic tasks differ from everyday prospective memory in important ways. Naturalistic tasks still provide instructions and structure, which may represent a form of 'scaffolding' that is particularly helpful for older adults (Craik, 1983). Everyday tasks rely heavily on self-initiated action, which becomes more challenging with age due to motivational influences and cognitive changes (Lindenberger \& Mayr, 2014). Thus, while older adults may show a surprising distinction between naturalistic and lab-based prospective memory tasks that demands ongoing investigation, this population still reports difficulties in daily life, which warrants attention and perhaps intervention.

\section{Evidence for episodic future thinking as a mnemonic aid for prospective memory: implementation intentions}

Although connections between memory and episodic future thinking are rather recent, the latter has been associated with goal achievement and motivation for decades. For instance, under the term mental simulation, episodic future thinking has been studied as a technique that can improve goals such as achieving better exam scores in university students (Taylor et al., 1999) and carrying out specific health behaviours (Greitemeyer \& Wuerz, 2006). Developed within the 
action phase model, Gollwitzer (1999) proposed "implementation intentions" as a self-regulatory technique to aid goal striving within the preactional phase. Implementation intentions go above and beyond mere goal intentions by specifying where, when and how one will carry out a future, goal directed behaviour: "If situation X occurs, then I will initiate goal-directed behaviour Y!" (Faude-Koivisto et al., 2009). In other words, implementation intentions involve linking a cue and a response using an if-then or when-then format (Faude-Koivisto et al., 2009).

Because prospective memory can be considered as a type of goal achievement task (e.g., Chen et al., 2015; Penningroth \& Scott, 2007), researchers have begun to apply techniques such as implementation intentions to improve performance. Forming implementation intentions forces the individual to explicitly specify critical components of the task to be remembered. Most of these studies have been carried out with younger adults, but a small number have explored the effects on lab-based tasks with older adults and have found positive results (Burkard et al., 2014; Chasteen et al., 2001; Liu \& Park, 2004; McFarland \& Glisky, 2011; Zimmerman \& Meier, 2009). For example, Liu and Park (2004) used implementation intentions to improve monitoring blood glucose levels, a naturalistic prospective memory task, which was successful. Burkard and colleagues (2014) designed a pilot study to teach older adults how to use implementation intentions (with both verbal and imagery components) to improve everyday prospective memory and other types of goal-directed behaviour, with promising results. Thus, there is evidence to suggest that prospective memory performance can be improved in and outside the lab using implementation intentions. The possible mechanisms through which this improvement occurs are reviewed in a later section.

What remains unclear is whether implementation intention effectiveness is improved with the addition of an imagery component. Cohen and Gollwitzer (2008) claim that implementation 
intentions are not enhanced by the addition of imagery. Likewise, McDaniel et al. (2008) found that imagery encoding did not improve performance above baseline, but Meeks and Marsh (2010) and Brewer and colleagues (2011) found that it did. Similarly, it is unclear if and how implementation intentions and episodic future thinking differ from each other with respect to effects on prospective memory performance. There have been only a handful of studies comparing both methods to each other. McFarland and Glisky (2012) compared them in younger adults and found that implementation intentions, imagery, and the combination of the two each significantly improved performance as compared with a read-only condition, but each of the three experimental conditions did not differ from each other. In Chen et al.'s (2015) metaanalysis, implementation intentions were found to have a medium effect size in improving prospective memory in younger adults $(d=.45)$ and a medium-large effect size in older adults $(d$ $=.68)$. In all but one of the ten studies with older adults, the verbal and imagery components were combined, making it impossible to determine their individual contributions.

\section{Evidence for episodic future thinking and prospective memory improvement}

Older adults. To our knowledge, only two studies have applied a pure episodic future thinking intervention to improve prospective memory, only one of which included older adults. As mentioned earlier, Altgassen et al. (2015) found that episodic future thinking benefited prospective memory performance in older adults using the Dresden Breakfast Task. Older and younger adults were either required to imagine themselves completing the task at encoding, or simply follow the standard instructions. As expected, the older adults performed significantly worse than the younger adults, but most importantly, both groups improved equally as a result of the future thinking manipulation. This suggests that adults across the lifespan can glean similar benefits from future thinking. 
Likewise, Neroni, Gamboz and Brandimonte (2014) had younger adults mentally simulate completing a prospective memory task that they would return to the lab to complete the following day. In this task, participants viewed verbs and had to decide which had double consonants (ongoing task) while remembering to press a target key whenever any of the four cue words appeared (prospective memory task). Results showed that even after this 24-hour delay between future thinking and the prospective memory task, performance was significantly improved compared with the control condition.

Despite the fact that there have been so few studies using episodic future thinking to improve prospective memory, several studies with older adults have applied implementation intentions with an episodic future thinking (imagery) component. These studies provide some evidence for a favourable effect of the combined strategy. Schnitzspahn and Kliegel (2009) tested implementation intentions with imagery in young-old and old-old healthy adults using a lab-based prospective memory task adapted from Chasteen et al. (2001). They found that the strategy improved performance for the young-old group only; in fact, the manipulation hurt performance on the event-based task in the old-old adults. Burkard and colleagues (2014) tested older adults from a memory clinic and found that only those with normal working memory capacity benefitted from the implementation intentions with imagery. Thus, it appears that imagery might have a role to play but that its effect depends on participant characteristic such as age and cognitive capacity.

Clinical populations. Additional evidence can be drawn from several other studies that have employed episodic future thinking to improve prospective memory performance in other populations that demonstrate deficits in this domain. Within the alcohol addictions literature, a small number of studies have applied an episodic future thinking intervention during the 
encoding phase of the prospective memory task and overall, the effect size of this strategy across these studies is small-medium (Faytell et al., 2016). For example, Leitz, Morgan, Bisby, Rendell, and Curran (2009) studied the effects of acute alcohol ingestion on prospective memory utilizing the Virtual Week task (Rendell \& Craik, 2000; Rendell et al., 2009), which is a laboratory-based prospective memory task that has relatively high ecological validity ${ }^{11}$. In this task, participants move a virtual token around a computerized board game and must complete event- and time-based, regular and irregular prospective memory tasks while performing everyday tasks during a series of virtual days. Participants perform the regular (time and eventbased tasks) every day, while the irregular tasks change with each new virtual day. Using a within-subjects design, the authors compared the standard instructions (i.e., verbally rehearsing the instructions for the day's prospective memory tasks aloud) with a mental simulation task, which required participants to imagine carrying out the task for 10 seconds, for the irregular tasks only. Interestingly, in the simulation condition, participants were instructed to imagine doing the tasks in their everyday lives (e.g., if picking up something from the grocery store at a certain time was the irregular task for the day, participants should imagine going to the store where they usually shop). Of interest here, the findings suggested that the simulation instructions improved performance in the placebo/control (versus alcohol) group for the irregular, event-based tasks. The authors speculated that perhaps the alcohol prevented participants from fully engaging in the future thinking strategy, or that for some unknown reason, it interrupted any advantage the strategy might confer.

In a related study, Paraskevaides et al. (2010) again used the Virtual Week task to explore how episodic future thinking might mitigate the detrimental effects of acute alcohol ingestion.

\footnotetext{
${ }^{11}$ This task is used in Experiment 2 of this dissertation and is described in the following chapter.
} 
The manipulation was similar although they made minor changes to some parameters of the task. Nonetheless, they replicated the findings of Leitz et al. (2009), and showed that the future event simulation improved prospective memory performance for the irregular event-based tasks, improved performance for participants in the placebo condition, and eliminated the prospective memory impairment caused by the acute alcohol. The authors suggest that the future episodic simulation improved prospective memory by strengthening "the intention with a specific visualspatial context." As such, cues would be detected more automatically, which would facilitate task completion.

More recently, Griffiths and colleagues (2012) employed a similar paradigm comparing individuals who met criteria for alcohol dependence with social drinkers. Here, episodic future thinking improved performance for time-based prospective memory tasks for social drinkers but not for those with alcohol dependence. The authors speculated that these individuals may not be able to benefit from the strategy due to troubles with planning rather than imagining skills per se since there were no differences in self-reported vividness between groups. One limitation was that the future episodic simulations might have improved performance simply because participants had more time to encode the instructions rather than as a result of the simulation per se. Finally, Platt, Kamboj, Italiano, Rendell, and Curran (2016) used a similar paradigm with heavy drinkers versus control participants. Overall, the heavy drinkers had poorer performance on time-based tasks compared with the control participants. Future thinking improved performance on time-based tasks only for control participants and improved performance on event-based tasks only for the heavy drinkers. Taken together, there is evidence that future thinking can be utilized by healthy individuals as well as those experiencing deficits in 
prospective memory, although the exact patterns across event- and time-based tasks is inconsistent, perhaps due to slight differences in paradigms and sample characteristics.

Episodic future thinking has also been applied as mnemonic aid for individuals suffering from neurological damage. Grilli and McFarland (2011) showed that the strategy significantly improved prospective memory performance in this population. In this study, the ongoing task consisted of general knowledge multiple choice questions and the prospective memory task required pressing ' 1 ' in response to specific target words. Compared with a rote-rehearsal encoding condition, the self-imagination condition significantly improved performance (66\% advantage) and did not negatively impact performance on the ongoing task. The authors propose that this type of self-imagining or mental simulation not only relies on episodic memory function but also incorporates information from the semantic knowledge of the self, related to the first two levels of the self-memory system described earlier, which may be preserved in these individuals.

\section{Methodological limitations and outstanding questions}

In summary, it appears that prospective memory might benefit from an episodic future thinking intervention, but the literature still contains outstanding questions and methodological caveats. The review by Chen et al. (2015) indicated that only one study compared the verbal and visual forms of implementation intentions in younger adults (McFarland \& Glisky, 2011) and did not find a significant difference. Because so few studies have actually compared the verbal form of implementation intentions with episodic future thinking, it is unclear if it is the act of firstperson mental simulation that is responsible for the improvements in prospective memory performance. Most studies only compare the future thinking condition with standard instructions, which differ in length of encoding time, representing a significant confound. Many 
studies do not include a baseline condition, making it difficult to draw conclusions about mechanisms of these strategies (Chen et al., 2015). Other factors such as motivation or the reprioritization of the ongoing versus the prospective memory task were not assessed, although these factors are critical for determining mechanisms of these interventions. Finally, few studies have explored episodic future thinking as an intervention in older adults; thus replication is needed.

More broadly, there are also important methodological considerations in the prospective memory literature concerning the cognitive aging paradox. As already indicated, many studies have not assessed motivational differences between laboratory and naturalistic tasks, which might help account for the paradox. Finally, many prospective memory tasks provide participants with very few opportunities to carry out their prospective memory activity. For example, lab tasks may have four targets (i.e. cues), making them relatively insensitive measures of prospective memory. From a reliability perspective, tasks with stronger psychometric properties should also be utilized to better understand the paradox (Rendell \& Henry, 2009).

\section{Possible mechanisms of action}

As described in the previous section, the research is inconclusive with respect to whether and how episodic future thinking benefits prospective memory and how it might compare with verbal implementation intentions. Nevertheless, several candidate mechanisms have been put forward. From a social psychology perspective, Taylor et al. (1998) suggest that mental simulation benefits goal achievement by refining problem solving and planning, or by regulating affect and emotional states. Both implementation intentions and episodic future thinking force deeper thinking about how the future activity may be achieved. This may lead to the discovery of potential obstacles or other considerations that would otherwise remain hidden if the instructions 
were accepted at face value. Others have suggested that mentally simulating a future behaviour might improve feelings of self-efficacy, which would increase motivation and hence performance (Bandura, 1986; Lock \& Latham, 1990; Taylor et al., 1998).

Researchers contributing to the literature on implementation intentions and goal achievement (e.g., Faude-Koivisto et al., 2009; Gollwitzer, 1999) hypothesize that implementation intentions and episodic future thinking represent distinct self-regulatory techniques that should be beneficial for prospective memory, albeit via separate mechanisms. Both are relevant in the pre-actional phase and promote action planning. Implementation intentions force the selection of a specific future situation (i.e., a cue) that is activated and accessible such that sensitivity to the cue is enhanced and goal responding is improved via automatic processes. In contrast, future thinking allows for the exploration of several possible plans in a more flexible manner. It is suggested that episodic future thinking should be most beneficial if performed first, in order to explore all options, whereas the implementation intention should be performed subsequently in order to "lock down" the plan (Faude-Koivisto et al., 2009). Thus, benefits should occur at different time points along the goal completion process, but this hypothesis awaits empirical validation (Faude-Koivisto et al., 2009).

By contrast, other researchers conjecture that the mechanisms associated with either strategy do not differ from each other. Altgassen et al. (2015) propose that episodic future thinking strengthens cue-action associations; that is, it strengthens encoding. As a result, it should benefit prospective memory performance via mostly automatic processes, just as implementation intentions are alleged to do. Meanwhile, others have suggested that both strategies might implicitly shift resource allocation to the prospective memory task, representing more controlled processes (Chen et al., 2015). That is, it is possible that motivation is the 
mechanism responsible for the improvements in prospective memory performance. It is also possible that both controlled and automatic processes are implicated. Burkard et al. (2014) suggest that because working memory is required for these strategies to be effective, it follows that some controlled processes are involved, even if the benefits occur mainly through automatic processes.

Still others have sidestepped the automatic/controlled debate and wondered if the "selfimagining" (i.e., episodic future thinking) is beneficial due to self-referential processing (e.g., Grilli \& McFarland, 2011). Indeed, since the 1970s (see Klein, 2012, for a review), it has been shown that introducing self-referential processing into the encoding phase significantly improves memory performance. Grilli and McFarland (2011) suggested that imagining the self performing the future task activates thoughts and feelings about the self, which can later be used as accessible retrieval cues for the prospective memory task. Further, they suggest that even individuals who have mnemonic deficits - such as those with neurological damage included in their studies, and relevant here, older adults - can benefit from this strategy since this general, more semantic self-relevant knowledge (i.e. the top two levels of the SMS) is usually still intact and thus could be used even if these individuals have trouble generating specific future episodic events. Indeed, this is congruent with the fact that in Altgassen et al.'s (2015) study, both older and younger adults improved similarly despite the fact that older adults are known to have trouble with episodic future thinking. In summary then, it remains unclear if and how episodic future thinking aids prospective memory and if its effects differ from related strategies such as implementation intentions. Nonetheless, it represents a promising technique for improving a cognitive domain that is often a concern for older adults. 


\section{Chapter 5: Experiment 2}

The previous chapter introduced the concept of prospective memory and reviewed the literature describing the associated age-related changes, including the differential patterns observed between lab-based and naturalistic tasks. Overall, older adults appear to experience difficulties with prospective memory both in the lab and in tasks of daily living. As such, prospective memory represents a key target for interventions. Implementation intentions are one such strategy, with limited but promising evidence across various populations, including older adults. Research from the alcohol dependence literature was also reviewed, which suggests that episodic future thinking may represent another promising strategy to improve prospective memory, although the mechanisms of action remain to be elucidated. Added support for the utility of episodic future thinking comes from theories of prospection (e.g., Szpunar et al., 2014), which predict that it may also improve prospective memory performance. One study has been conducted to test this claim in older adults (Altgassen et al., 2015) with positive results, although methodological limitations were noted. In the second dissertation experiment, we sought to extend this work.

\section{Rationale and Hypotheses}

In order to test hypotheses about the function of episodic future thinking, it was used as a strategy during the encoding phase of a lab-based prospective memory task. Older adults in particular have difficulties with such tasks and so determining if such a strategy could be effective would be of benefit to this population. The prospective memory task chosen for this experiment is the Virtual Week (Rendell \& Craik, 2000). As a reminder, this task involves clicking a virtual die and moving a token around a board displayed on a computer screen, with one circuit of the board representing one virtual day. A clock is displayed on the centre of the 
screen to mark the advancing time of day. As participants circuit the board, they are prompted to draw cards from an Event pile. Each card describes a specific daily activity (e.g., "you go to your exercise class") that the participant virtually completes. In addition, participants are assigned 'regular' tasks at the start of each day that they must remember to perform; they are deemed 'regular' because these same tasks are assigned and performed every virtual day. Participants are also assigned 'irregular' tasks throughout the day that they must remember to perform. These tasks are classified as 'irregular' because they represent a new set of tasks each day. Both regular and irregular tasks comprise two types, depending on the cue, which may be time-based - for example, "take asthma medication at 11 am" - or event-based, such as "take antibiotics with breakfast."

One of the attractive features about the Virtual Week is that it balances standardization with ecological validity. The trials in the Virtual Week are chosen to mimic actual tasks of daily living, making them more meaningful to participants. This is in contrast to many other prospective memory tasks wherein participants must press a key when a particular word pops up on the screen in the context of another task. The Virtual Week also comprises many more trials than other prospective memory tasks and has been used across a variety of studies, all of which contribute to greater reliability. Moreover, because one of the only other studies that employed an episodic future thinking manipulation to improve prospective memory in older adults (Altgassen et al., 2015) used a conceptually similar task (i.e., the Dresden Breakfast Task), using the Virtual Week here allowed us to conduct a conceptual replication. The Dresden Breakfast Task also involves performing event- and time-based tasks in an ecologically valid setting: participants must prepare breakfast for four people using a computer simulation. However, it 
differs from the Virtual Week in that there is a heavier emphasis on planning, which may make for a less pure test of prospective memory.

The psychometric properties are another valuable feature of the Virtual Week. Testretest reliabilities of prospective memory tasks are low, often estimated at $20 \%$ (e.g., Keleman, Weinberg, Alford, Mulvey \& Kaeochinda, 2006; Rendell \& Henry, 2009). McDaniel and Einstein (2007) attribute this to the fact that most prospective memory tasks involve very few targets. In contrast, the Virtual Week includes many more targets and is associated with reliabilities ranging from .84 to .94 in healthy controls, depending on the type of target (as described in Rendell \& Henry, 2009). Aging studies using the Virtual Week demonstrate that age differences are small for the regular tasks but significantly larger for the irregular tasks (e.g., Rendell \& Henry, 2009).

Returning to the current experiment, the central aim of this experiment was to investigate if episodic future thinking is a beneficial strategy for prospective memory performance using the Virtual Week. To address the methodological problem of longer encoding period in earlier studies testing this strategy, it was compared with a control strategy, implementation intentions, in addition to a no-strategy condition, which served as a baseline. Contrasting episodic future thinking with implementation intentions allowed us to answer calls in the literature for a direct comparison between the two strategies (e.g., Chen et al., 2015).

Regarding specific hypotheses, a main effect of age was expected, with younger adults showing better prospective memory performance on all types of tasks (event-based and timebased). Age differences should also emerge for the regular and the irregular tasks, with younger adults outperforming older adults for both regular and irregular tasks (e.g., Rendell \& Henry, 2009). With respect to the effect of the strategies, the predictions were less certain. It was 
hypothesized that both strategies would improve both event- and time-based prospective memory performance on the irregular tasks ${ }^{12}$, but it was unclear how they would compare with each other or if any age by condition interactions would emerge. Based on a recent review (Chen et al., 2015), both age groups were expected to benefit from implementation intentions compared with baseline, since previous studies have shown it to be a beneficial strategy for both younger and older adults. Regarding episodic future thinking, however, a clear prediction was more difficult to make. In the alcohol use literature reviewed in the previous Chapter, some studies found that episodic future thinking improved performance on the time-based tasks (e.g., Griffiths et al., 2012; Leitz et al., 2009;) while others found it improved performance on the event-based tasks (Paraskevaides et al., 2010); that is, a consistent pattern failed to emerge across studies.

Drawing from the cognitive aging literature on episodic future thinking, younger adults' prospective memory performance might benefit more than that of older adults since older adults tend to struggle with episodic future thinking. In this case, an interaction between age and condition would be observed. On the other hand, Altgassen et al. (2015) found that older adults' performance improved just as much as younger adults' performance, suggesting that older adults can utilize future thinking in the same way as younger adults. One possibility is that because the future thinking task is much more structured and directed in this type of experiment (i.e., it is very clear what the older adults should imagine) than typical future-thinking paradigms (e.g., future thinking as a function of a cue word, which is akin to free recall), older adults can engage in future thinking similarly to younger adults. In other words, the structured nature of the task may provide sufficient cognitive scaffolding for the older adults such that they are able to engage

\footnotetext{
${ }^{12}$ There should be no effect of either strategy on the regular tasks since they are repeated each day and the strategies are not applied to them.
} 
in similar levels of future thinking as the younger adults. Overall, this is an empirical question that was tested in this experiment.

\section{Methods}

Participants. Thirty-six (28 female) younger adults aged 17-23 years $(M=19.03, S D=$ $1.50)$ and 36 older (26 female) adults aged $65-85$ years $(M=72.58, S D=5.68)$ completed the study. This sample size is based on our previous work with younger and older adults and provides sufficient statistical power $(\geq .90)^{13}$ to detect medium-sized between- and withingroup main effects and interactions. The younger adults were students enrolled in introductory psychology classes at Ryerson University and received course credit for their participation. The older adults were recruited over the phone from Ryerson's database of older adults who have indicated their interest in being contacted for participation in research studies. They received $\$ 30$ each for their participation. Participants were healthy and included only if they met the following criteria, which was established via interview with an experimenter: no current or past neurological disease, no learning disabilities, no brain tumour, no other current or past (within the past 3 years) diagnosis of cancer, no current psychiatric diagnoses, no uncontrolled thyroid, metabolic, or cardiovascular disease, and no vision or hearing problems (unless corrected to normal). In addition, older adults were included only if they scored higher than 26 on the MMSE (Folstein et al., 1975) to screen for cognitive impairment. Finally, participants were excluded if they were not fluent English speakers.

Procedure. After providing written informed consent to participate, participants began the Virtual Week (Rendell \& Craik, 2000) to test their prospective memory. All participants performed a practice "day," as is typical for this task. Subsequently they completed one

\footnotetext{
${ }^{13}$ Established using G*Power 3.1 with alpha $=.05$. Effect sizes used were large for the between-subject tests and medium for the within-subjects and interactions calculations.
} 
"control" day with the standard instructions. Next, they completed two more days: one with the future thinking instructions and one with the implementation intention instructions, and the order of the instructions was counterbalanced across participants. Prior to beginning either experimental condition (i.e., the future thinking days or the implementation intention days), the Virtual Week was stopped so that participants could run through a brief, computerized tutorial presenting the instructions. The tutorial involved an explanation, an example, and two practice trials during which the participants were asked to implement the encoding instructions (see the Appendix for the tutorial slides viewed by participants). Feedback from the experimenter was provided as needed. Once the tutorial was completed, participants returned to the game and applied the encoding instructions for another virtual day.

In the original procedure, participants were expected to play two virtual days per condition. However, the older adults performed the task more slowly than expected during piloting and many would have exceeded the session time limit. As such, it was decided that participants could play only one virtual day per condition.

The future thinking instructions were modeled after Leitz et al.'s (2009) protocol, in which participants imagined themselves carrying out the virtual day's irregular tasks in their everyday lives. Participants had 30 seconds to describe their future thinking narratives out loud. If they spoke for less than 20 seconds, the experimenter provided one prompt ("Is there anything else you can imagine about this scenario?"). For the implementation intention instructions, participants were told to form a simple verbal statement with no imagery component. That is, they were told that they should not employ any visualization strategy and should only focus on the verbal statements they were making. Following Burkard and colleagues lead (2014), this set of instructions was referred to as the "When-Then" (WT) strategy with participants since this 
terminology is simpler than "implementation intentions." To match the time spent on encoding in the future thinking condition, when participants were in the implementation intentions condition, they were asked to repeat their statement aloud four times, which corresponded to a similar amount of time.

Once participants completed the Virtual Week, they returned to the table where they completed a battery of baseline measures: Verbal Paired Associates I and II (taken from the Wechsler Memory Scale, Fourth Edition; WMS-IV; Wechsler, 2009), the DASS-21 (Lovibond \& Lovibond, 1995), the Shipley Vocabulary Test (Shipley, 1940), Digit Span (taken from the Wechsler Adult Intelligence Scale, Fourth Edition; WAIS-IV; Wechsler, 2008), Trails A and B (Reitan \& Wolfson, 1985), and the Vividness of Visual Imagery Questionnaire (VVIQ; Marks, 1973).

Of note, the younger adults received a demographic questionnaire once the battery was completed, whereas the older adults were administered the MMSE (Folstein et al., 1975). The older adults had already completed a demographic interview over the phone during the recruitment process. All participants were asked if they had a preference for either of the encoding strategies or not. Finally, participants were debriefed about the purposes of the experiment and compensated for their participation.

\section{Materials}

The Virtual Week task. This is a well-validated measure of prospective memory (Rendell \& Craik, 2000), with good psychometric properties and sensitivity to pathology as well as healthy age-related changes in older adults (Rendell \& Henry, 2009). Rose and colleagues (2010) found that across their sample of healthy younger and older adults, reliability estimates ranged from .84 to .94 for the regular, irregular, and time-check tasks. Split-half reliability has 
been estimated to be .74 in a group with schizophrenia and .66 for the healthy controls (Henry et al., 2007). In the context of aging, decline associated with increasing age is small on regular tasks, but "substantial" on irregular tasks (reviewed in Rendell \& Henry, 2009). Rose and colleagues (2010) reported a main effect of age on the Virtual Week, with an effect size of $\eta_{p}{ }^{2}=$ .53 , and follow-up analyses associated with an effect size of $\eta_{p}{ }^{2}=.59$ for the age difference on the irregular tasks, compared with $\eta_{p}{ }^{2}=.38$ on the regular tasks. Recently, Mioni and colleagues (2015) confirmed that internal consistency was high for younger and older adults and that testretest reliability was also high.

For each virtual day, participants "move" a token around the board (see Figure 6) by clicking a virtual die to determine spaces moved. The passing hours of the day are continuously shown on a clock at the centre of the board. Each circuit of the board represents one day. As participants move around the board, they are directed to draw from an Event card pile, which requires them to make decisions (e.g., what they should eat for lunch) or perform certain activities (i.e., select the activity from a drop-down menu of possible activities); this represents the ongoing task. Participants must complete regular event- and time-based prospective memory tasks every day, such as taking a medication at a particular time and performing another activity after breakfast. The time-based tasks are cued by the clock (e.g., take asthma medication at 11 am and $9 \mathrm{pm}$ ) on the screen, whereas the event-based tasks (e.g., take antibiotics after breakfast and dinner) are cued by draws (e.g., of breakfast and dinner cards) from the Event cards pile.

Participants must also complete new, irregular tasks each day. At the start of each new day, participants read aloud a start card that indicates the two irregular tasks for the day. Each new day brings ten prospective memory tasks in total: four regular, four irregular, and two timecheck tasks (see Figure $\mathbf{7}$ for a visual depiction of how the regular and irregular tasks are 
distributed within and across "days" of the Virtual Week). In the current study, the critical manipulation (future thinking vs. implementation intention vs. control) is applied only to the irregular tasks since these are different each day. The manipulation could not be applied to the regular tasks, which are prone to practice effects because they are repeated each day.

To perform each task, participants click a specific box on the screen that allows them to choose from a drop-down menu which task they wish to perform at that time (based on the time displayed on the clock), or in response to an event-based cue. Participants go through a practice day first to learn the components of the task, after which they complete three days (i.e., baseline instructions, followed by future thinking or WT, depending on counterbalancing). Three days of the Virtual Week corresponds to a total of 30 prospective memory tasks in total.

\section{Baseline Measures}

The Depression Anxiety and Stress Scale 21 (DASS-21; Lovibond \& Lovibond, 1995):

The DASS-21 measures and discriminates between the constructs of depression, stress, and anxiety. In this version, participants answer 21 questions about somatic and psychological symptoms of these three constructs over the past week using a 0 to 3 rating scale. It has been found to distinguish reliably between depression, physical arousal, and psychological tension and agitation (Antony, Bieling, Cox, Enns, \& Swinson, 1998). Moreover, the internal and concurrent validity were found to be in the acceptable to excellent range (Antony et al., 1998). In a sample of older adults specifically, the three-factor structure was replicated, along with evidence for good internal consistency, excellent convergent validity, and good discriminative validity (Gloster et al., 2008).

The Trail Making Test (TMT; Reitan \& Wolfson, 1985): The TMT measures visual search, scanning, processing speed, and executive functions (Tombaugh, 2004). The measure 
consists of two parts, A and B. In part A, participants must connect 25 numbers that are placed randomly on a page with a pencil, as fast as possible. In part B, the task difficulty is increasing by requiring participants to switch between numbers and letters, connected each in ascending order (e.g., 1, A, 2, B, 3, C, etc.). Scores are measured in time taken to complete the task. As such, lower scores reflect faster, and hence, better performance. According to one study (Sellers $\&$ Nadler, 1992), it is the second most widely used neuropsychological measure in patients 60 years of age and older. As summarized in Bowie and Harvey (2006), it has been found to have excellent interrater reliability but is susceptible to practice effects for short intervals (e.g., 6 weeks; Stuss, Stethem, \& Poirier, 1987) but likely not after 6 months (Giovagnoli et al., 1996).

Verbal Paired Associates I and II (from the Wechsler Memory Scale IV, WMS-IV; Wechsler, 2009): These subscales provide an index of immediate and delayed verbal memory for associated word pairs, respectively. Participants are read a series of word pairs, after which the test period begins, where the examiner reads one word at a time and the participant must recall the corresponding word. There are four trials of the same list in a different order. In part II, participants are tested on their memory for the word pairs in both a recall and recognition format.

The WMS-IV is among one of the most popular measures employed by neuropsychologists (e.g., Butler, Retzlaff, \& Vanderploeg, 1991) and has been considered the gold standard for memory evaluation (e.g., Hartman, 2009).

Vividness of Visual Imagery Questionnaire (VVIQ; Marks, 1973): This is a 16-item self-report questionnaire asking participants to visualize and rate vividness of mental imagery on a 5-point scale. Lower scores indicate more vivid imagery. Although the relationship to objective measures of imagery are low, the VVIQ is still one of the most commonly used 
subjective measures of visual imagery and is related to performance in visual memory experiments (e.g., Marks, 1973). The VVIQ has also demonstrated acceptable split-half and internal consistency (McKelvie, 1986). It has also been found to be unifactorial (Richardson, 1999). D'Argembeau and Van der Lindsen (2006) found that the VVIQ significantly predicted vividness and other phenomenological characteristics for both autobiographical memories and episodic future thinking.

Mini-Mental Status Examination (MMSE; Folstein, Folstein \& McHugh, 1975): This is one of the most widely used screening tools for general cognitive functioning (Folstein, Folstein, McHugh, \& Fanjiang, 2001). The 30-items are organized into the following domains: orientation, attention and calculation, recall, language, and a three-stage command. According to Folstein et al. (2001), the following cut-off scores are suggested: greater or equal to $27=$ normal; $21-26=$ mild; $11-20=$ moderate; less than $10=$ severe. Although criticized for low reliability and lack of standardized scores, it has been used since 1975 and remains a popular screening (if not diagnostic) tool (Lopez, Charter, Mostafavi, Nibut, \& Smith, 2005). Tombaugh and McIntyre (1992) found that measures of reliability and validity were satisfactory and support its use as a brief screening measure that can be used to document change over time but should not by itself be used to make diagnostic judgments.

\section{Analyses}

All data analyses were carried out with IBM SPSS Statistics Version 23. The results from the Virtual Week were analyzed according to the instructions provided by the task developers (Rendall \& Craik, 2000), as well as following the precedent set by previous studies utilizing the task (e.g., Leitz et al., 2009). The main variable of interest was the mean proportion of correct responses for the regular and irregular tasks, as well as for task type (event-based or 
time-based). Responses were considered correct if the correct action was performed at the correct time (e.g., if the assigned task was "Get a haircut at 12 pm", the response would be considered correct if the 'haircut' task was selected from the drop-down menu when the virtual clock displays $12 \mathrm{pm}$ ) or before the next dice roll. Response times per se are not significant because the virtual clock only moves when the participant manually moves the token from one square to the next; that is, the clock does not correspond to real time. As such, the Virtual Week is self-paced and is not affected by individual differences in response time.

The Virtual Week data were submitted to mixed $2 \times 3 \times 2 \times 2$ ANOVA, with age (2 levels: older and younger) as the between-subjects factor, and condition (3 levels: baseline, future thinking instructions, and implementation intention instructions), task type (2 levels: event-based or time-based tasks) and regularity (2 levels: regular or irregular) as the withinsubjects factors. Any interactions were explored with further ANOVAs and post-hoc, Bonferroni-adjusted pairwise comparisons. Relationships with the baseline neuropsychological measures and other sample characteristics were explored with Pearson correlations.

\section{Results}

Participant characteristics. The results from the neuropsychological battery are summarized in Table 2. In brief, older adults demonstrated significantly higher levels of education and crystallized intelligence and lower levels of depression, stress and anxiety, as captured by the DASS-21. Younger adults outperformed the older adults in the domains of executive function and associative memory, both immediate and delayed. Age groups did not differ in working memory performance or self-reported vividness of visual imagery (VVIQ). Data from one older adult were unavailable for the VVIQ because she had to leave the testing session early. 


\section{The Virtual Week.}

Performance on the Virtual Week task is summarized in Table 3. A 2 (age: younger vs. older) x 3 (condition: baseline vs. FT vs. WT ${ }^{14}$ ) x 2 (task type: event vs. time) x 2 (regularity: regular vs. irregular) mixed ANOVA was performed. Performance was expected to improve after baseline, for both regular (due to practice effects) and irregular (due to condition) tasks, which would be consistent with previous studies (e.g., Rendell \& Craik, 2000). However, a Condition x Regularity interaction was expected since the use of strategies (condition) was predicted to improve performance on the irregular tasks to a greater extent. Additional predictions, based on previous studies, included better performance on event- versus time-based tasks and that younger adults would perform better than older adults. A significant Task type $\mathrm{x}$ Age interaction was not predicted to emerge.

The results showed main effects of Condition, $F(2,140)=8.48, p<.001, \eta_{p}{ }^{2}=.11$, Task type, $F(1,70)=36.96, p<.001, \eta_{p}{ }^{2}=.35$, Regularity, $F(1,70)=32.34, p<.001, \eta_{p}{ }^{2}=.32$, and Age, $F(1,70)=10.27, p=.002, \eta_{p}{ }^{2}=.13$. As predicted, performance was higher on the regular tasks as compared with the irregular tasks, and event-based task performance was better than time-based task performance. Regarding strategy use, baseline performance was worse compared with the FT $(p=.001)$ and the WT $(p=.014)$ conditions, while the latter two did not significantly differ from each other. Finally, also as expected, younger adults outperformed the older adults.

Only one significant interaction emerged, Regularity x Task type, $F(1,70)=7.31, p=$ $.009, \eta_{p}{ }^{2}=.10$. On average, event-based task performance was higher on regular $(M=.74, S E=$ $.03)$ as compared to irregular tasks $(M=.66, S E=.04)$. Similarly, time-based task performance

\footnotetext{
${ }^{14}$ As a reminder, the implementation intentions condition/strategy was called "When-Then" for simplicity with participants (Buckard et al., 2014).
} 
was higher when they were regular $(M=.63, S E=.03)$ than when they were irregular $(M=.42$, $S E=.03)$. Nonetheless, compared with event-based tasks, performance on the time-based tasks was impacted to a greater extent by regularity; the time-based tasks were associated with a greater change in performance as a function of regularity. These results were followed-up with ANOVAs separated by regularity ${ }^{15}$. Surprisingly, there were no interactions between Regularity and Condition, or between Regularity and Age, which was contrary to predictions and previous studies, as reviewed earlier.

Regular Tasks. A 2 (age: younger vs. older) x 3 (condition: baseline vs. FT vs. WT) x 2 (task type: event vs. time) mixed ANOVA revealed a significant effect of Age, $F 1,70)=4.71, p$ $<.05, \eta_{p}{ }^{2}=.06$, Condition, $F(2,70)=10.55, p<.001, \eta_{p}{ }^{2}=.13$, and Task type, $F(2,70)=11.16$, $p=.001, \eta_{p}{ }^{2}=.14$, but no significant interactions. Regarding the main effect of Age, younger adults $(M=.74, S E=.04)$ significantly outperformed older adults $(M=.63, S E=.04)$. Unpacking the main effect of Condition, performance for both the FT $(M=.74, S E=.03 ; p<$ $.001)$ and WT $(M=.72, S E=.03 ; p=.004)$ strategies was significantly higher than baseline $(M$ $=.60, S E=.03)$ but that they did not differ from each other. Finally, as expected, performance was significantly higher on the event-based tasks $(M=.74, S E=.03)$ as compared to the timebased tasks $(M=.63, S E=.03 ; p=.001)$. These results suggest that as expected, performance on the regular tasks improved with repeated exposure and that strategy introduction did not hinder this improvement (see Figures 8 and 9).

Irregular tasks. A new set of irregular tasks, two event-based and two time-based, were introduced during each day of the Virtual Week. Prior studies have shown that performance on

\footnotetext{
${ }^{15}$ In addition, the ANOVAs were separated by task type to be consistent with some previous studies, which treated the regular tasks like control tasks (since strategies were not applied to them and thus not expected to have an effect; Paraskevaides et al., 2010).
} 
irregular tasks is lower than on the regular tasks and that performance does not improve across subsequent days of the Virtual Week, presumably due to the novelty of the tasks (reviewed in Rendell \& Henry, 2009). A main effect of Age was predicted, with younger adults outperforming older adults on both event- and time-based tasks. The main outcome of interest however was the effect of Condition (i.e., strategy). We predicted that the FT and WT strategies would lead to improvement on the irregular tasks, but specific interactions were difficult to predict. Previous research, as reviewed earlier, is mixed regarding whether the age groups would respond differently to the different strategies.

The 2 (Age) x 3 (Condition) x 2 (Task type) mixed ANOVA showed a significant main effect of Age, $F(1,70)=10.89, p<.01, \eta_{p}{ }^{2}=.14$, and Task type, $F(1,70)=37.24, p<.001, \eta_{p}{ }^{2}$ $=.35$, which were both consistent with predictions. Most importantly however, there was no main effect of Condition, $F(2,140)=1.36, p=.26, \eta_{p}^{2}=.02$, contrary to the primary prediction. Following-up the two main effects, participants performed better on the event-based tasks $(M=$ $.66, S E=.04)$ than time-based tasks $(M=.42, S E=.03)$, and younger adults had higher scores $(M=.62, S E=.04)$ compared with the older adults $(M=.45, S E=.04 ;$ see Figures 10 and 11 for full summary). These latter two findings are consistent with predictions and with previous research. There were no significant interactions.

To summarize, the introduction of the strategies generally did not improve performance on the irregular tasks, which were the main outcomes of interest. The expected effects of Age and Task type (event- vs. time-based tasks) emerged, but there were no significant effects involving the strategies, which furthermore was associated with a small effect size of $\eta_{p}{ }^{2}=02$. In the first, omnibus test, the only interaction to emerge was between Regularity and Task type, due 
to time-based tasks being more sensitive to regularity than the event-based task. Expected interactions between Regularity and Age or between Regularity and Condition were not found.

\section{Secondary analyses.}

Correlations with cognitive and affective measures. Following prior studies suggesting relationships between certain neuropsychological variables and prospective memory performance (e.g., Rose et al., 2010), correlations were run within each age group to investigate how neuropsychological variables might relate to baseline Virtual Week performance. The only significant correlation was between immediate associative memory and regular time-based tasks at baseline, $r=.34, p<.05$, for younger adults only.

Similar correlations were explored for the subsequent days of the Virtual Week (i.e., the FT and WT conditions) for both regular and irregular tasks. Select correlations were found between certain neuropsychological variables and the time-based tasks only. In younger adults, irregular time-based performance (FT only) was negatively correlated with Trails A, $r=-.35, p<$ .05 , and Trails B, $r=-.43, p<.01$, respectively ${ }^{16}$. In older adults, Trails A but not B was correlated with irregular time-based performance (WT only), $r=-.38, p<.05$. Immediate associative memory was significantly correlated with irregular time-based performance under the FT, $r=.35, p<.05$, and WT conditions, $r=.42, p<.05$, respectively. Finally, delayed associative memory was correlated with irregular time-based performance (WT only), $r=.38, p$ $<.05$. If a Bonferroni correction for the high number of tests is employed, none of the correlations remain significant. While this type of correction is conservative, the fact that only

\footnotetext{
${ }^{16}$ For the Trail tests, because scores are measured in time, lower scores reflected faster and hence, better performance.
} 
one variable was correlated with baseline Virtual Week performance supports the possibility that the neuropsychological variables were not meaningfully related to Virtual Week performance.

Of note, the VVIQ did not correlate with irregular task performance in the FT condition, suggesting that subjective impressions of visual imagery ability were not associated with the effect of FT on prospective memory performance.

Strategy Preference. After the Virtual Week was completed, participants were asked if they preferred one of the strategies, both, or none at all. Responses were coded as follows: $1=$ FT preferred; 2 =WT preferred; $3=$ neither strategy preferred; $4=$ both were equally helpful. The pattern of responses differed between younger and older adults, $\chi^{2}(3, \mathrm{~N}=72)=14.22, p<$ .01. The response frequencies are reported in Table 4. For younger adults, the mode was 2, while for the older adults, the mode was 3. That is, most younger adults preferred the WT strategy while most older adults thought neither strategy was helpful.

A series of ANOVAs was run to explore relationships between strategy preference and performance on the irregular tasks of the Virtual Week. A 4 (strategy preference) x 3 (condition) x 2 (task type) mixed ANOVA was run across the whole sample. There was no main effect of Strategy preference or Condition, but there was a main effect of Task type, $F(1,68)=24.67, p$ $<.001, \eta_{p}{ }^{2}=.27$, due to higher performance on event-based tasks $(M=.65, S E=.04)$ compared with time-based tasks $(M=.42, S E=.04)$. More importantly, there was a significant Condition $\mathrm{x}$ Strategy preference interaction, $F(6,136)=3.22, p<.01, \eta_{p}{ }^{2}=.13$. ANOVAs run within each strategy preference group showed that the interaction was driven by those participants who preferred the FT strategy. These individuals showed a significant main effect of Condition, $F$ (2, $36)=7.08, p<.001, \eta_{p}{ }^{2}=.28$, and Task type, $F(1,18)=12.04, p<.001, \eta_{p}{ }^{2}=.40$, and no significant interaction. Follow-up pairwise comparisons for these individuals revealed that 
performance in the FT condition $(M=.75, S E=.07)$ was significantly better than baseline $(M=$ $.56, S E=.05)$ or in the WT condition $(M=.50, S E=.07 ; p<.05$ for both pairwise comparisons $)$, suggesting that individuals who prefer FT are able to significantly benefit from its utilization. All other strategy preference groups showed a significant main effect of Task type, as expected, but no effect of Condition and no interactions, indicating for these individuals, strategy preference had no effect on Virtual Week performance.

These analyses were repeated with age as a covariate (mean-centered as well as age uncorrected were tried $)^{17}$. The omnibus test remained unchanged, with a significant main effect of Task type and a significant Condition x Strategy preference interaction, as found originally. However, when this test was followed-up to understand the interaction, no main effect of Condition ${ }^{18}$ emerged for the FT group, $F(2,34)=1.47, p=.24, \eta_{p}{ }^{2}=.08$, in contrast to the earlier analyses without age as a covariate.

To explore relationships between strategy preference and performance on the battery of baseline neuropsychological tests, a series of univariate ANOVAs were run, first without age as a covariate, and then again using age as a covariate (mean-centered). Select tests showed significant differences based on strategy preference. When age was not entered as a covariate, performance on the Shipley was significant, $F(3,68)=6.06, p=.001, \eta_{p}{ }^{2}=.21$, explained by higher scores in the both-strategies group $(M=37.33, S E=2.02)$ compared with FT-preferred group $(M=30.11, S E=1.14)$ and the WT-preferred group $(M=29.96, S E=1.0)$. This pattern likely emerged due to uneven distribution of younger and older adults across the strategy

\footnotetext{
${ }^{17}$ Age was treated as a covariate, rather than exploring it by comparing age groups, because the number of participants for each strategy preference group was uneven, and this was significantly exacerbated when the sample was further divided by age group.

${ }^{18}$ Mean-centered covariate.
} 
preference groups. When age was entered as a covariate, there was no significant effect of strategy preference, $F(3,67)=1.51, p=.22, \eta_{p}{ }^{2}=.06$.

When age was not entered as a covariate, Trails A scores were significantly different, $F$ $(3,68)=2.96, p<.05, \eta_{p}{ }^{2}=.12$, but no pairwise comparisons were significant. When age was entered as a covariate, there remained a significant difference in Trails A scores, $F(3,67)=3.49$, $p<.05, \eta_{p}{ }^{2}=.14$, with the neither-strategy-preferred group showing slowest performance. This was significantly different than the both-strategy-preferred group. No other comparisons were significant.

In summary, while some differences emerged on some of the neuropsychological measures, they were not robust in the sense that most pairwise comparisons did not meet threshold for significance. Moreover, performance on measures that could be expected to relate to prospective memory performance - that is, indicators of executive function or memory - did not significantly differ between strategy preference groups, even when age was taken into account. Follow-up is required to test how robust the relationship between strategy preference and prospective memory performance is, especially given the small and unequal number of participants within each strategy group. Nonetheless, these results suggest a fruitful direction for future studies.

\section{Discussion}

As predicted, older adults demonstrated significantly poorer performance across conditions and task types of the Virtual Week, which is unsurprising given the wealth of literature supporting deficits in lab-based tasks of prospective memory as a function of age (Henry et al., 2004). Contrary to our main hypotheses however, the encoding strategy of FT did not confer any benefit on either type of prospective memory task (event- or time-based) 
performance. Moreover, there was a failure to replicate (e.g., Chen et al., 2015) any

improvement from implementation intentions on prospective memory performance. The results from the regular (repeated) prospective memory tasks improved with each day of the Virtual Week, despite the addition of the strategies. This improvement was expected, due to increased familiarity with repeated exposure to the tasks, which is consistent with all prior studies of the Virtual Week. This suggests that our participants did not behave any differently than those of other samples and provides confidence that the null findings with respect to the cognitive strategies were not due to some anomalous feature of our sample.

The failure to find any effect of FT or implementation intentions is surprising based on results from previous studies. A small but significant number of studies have suggested that FT aids prospective memory performance in children (Kretschmer-Trendowicz, Ellis \& Altgassen, 2016), adolescents (Altgassen et al., 2016), younger adults (McFarland \& Glisky, 2012; Neroni et al., 2014) and in older adults (Altgassen et al., 2015). Several studies with clinical populations have also found FT to improve prospective memory: those with HIV+ status (Faytell et al., 2015), individuals with various memory impairments (Grilli \& McFarland, 2011), and those with traumatic brain injury (Potvin et al., 2011). Studies using the Virtual Week in particular also suggest a positive effect for FT on this task. Research from the alcohol use literature, employing a similar paradigm to our study, has shown that FT can improve prospective memory in the Virtual Week. More specifically, Leitz et al. (2009) found FT enhanced prospective memory in the placebo group compared with those who ingested alcohol prior to the task. Paraskevaides et al. (2010) replicated these findings, showing improvement on both types of prospective memory tasks but a larger effect for event-based tasks, as well as for those in the acute-alcohol-ingestion group. Extending these findings beyond the effects of acute alcohol consumption to those with 
alcohol dependence, Griffiths and colleagues (2012) found that FT improved time-based prospective memory only in the control (social drinkers) group. Finally, Platt et al. (2016) found that FT improved event-based task performance only in a group of heavy drinkers, whereas FT improved time-based task performance only in the controls. These authors suggest that FT is helpful for the less demanding prospective memory tasks (event-based) but cannot offset the deficits in strategic monitoring and processing necessary for time-based tasks. Overall, the effect size of FT across these studies using various prospective memory tasks is small-medium (Faytell et al., 2016), which is generally larger than the non significant, main effect of condition found for the irregular tasks in our experiment, $\eta_{p}^{2}=.02$.

As regards implementation intentions, there is also evidence supporting a beneficial effect on prospective memory (see Chen et al., 2015, for a review). However, it should be noted that some studies have found mixed results (e.g., no main effects but some positive findings in secondary analyses; Belanger-Gravel, Godin, Bilodeau, \& Poirier, 2013) or even negative results for implementation intentions on prospective memory in older adults (e.g., implementation intentions hurt performance in the oldest-old; Schnitzspahn \& Kliegel, 2009). The reasons for the discrepant findings remain unclear and it should be noted that none had used the Virtual Week to test prospective memory. Schnitzspahn and Kliegel (2009) speculate that implementation intentions may have attentional costs that have not been measured or appreciated. As such, for particular populations that experience limited cognitive resources, incorporating implementation intentions may increase cognitive load and thereby hurt prospective memory performance. This may have been an important factor for our older sample. Prospective memory tasks that include a measure of how the ongoing task is impacted may 
represent one way to test this hypothesis. Unfortunately, the Virtual Week does not incorporate such a measure.

We can only speculate as to why our results diverge from prior work. Our design was more robust than the studies referenced earlier in that FT and implementation intentions were directly compared to each other in the same study. Few studies have conducted a direct comparison; most studies have employed one or the other or a combination. McFarland and Glisky (2012) directly compared implementation intentions and imagery, as well as both strategies combined, on a lab-based prospective memory task. This is the only study we are aware of that has conducted a direct comparison of these strategies. They found that both strategies were equally effective, and moreover, that the combination of the strategies did not demonstrate any additional benefit, suggesting that the strategies do not work synergistically. The authors concluded that the strategies likely rely on similar mechanisms; that is, they likely operate by strengthening intention formation and/or tapping into the encoding-specificity principle (Tulving \& Thomson, 1973) by increasing the degree of overlap between the simulated context at encoding and the context at retrieval (Brewer \& Marsh, 2010; Tulving \& Thomson, 1973). Because the current study employed a different prospective memory task, it is difficult to speculate further about why our results differed from theirs. Performance in our study was not at floor or ceiling and was comparable to performance in previous studies using the Virtual Week.

Of the studies employing the Virtual Week or analogous task (e.g., the Dresden Breakfast Task in the Altgassen et al., 2015, study) utilizing FT as an encoding strategy, none have included an active control strategy. On the one hand, this is a methodological strength of our study. On the other hand, it is possible that participants were overwhelmed by switching between three different conditions. Anecdotally, some older adults appeared to have difficulty 
just mastering the baseline and regular tasks, and appeared stressed by having to learn FT and implementation intentions. Another possible limitation is that by including three conditions, participants could only play one virtual day for each condition due to time constraints. Most other studies employing the Virtual Week have had at minimum of two virtual days per condition, which might make for a more reliable assessment. As regards the younger adults, perhaps performance across the sample was not sufficiently varied, or perhaps the task was not sufficiently demanding, to allow for any incremental benefit as a result of cognitive strategies. The younger adults in our study were not performing at ceiling, suggesting that sufficient room exists for improvement. Indeed, the studies of FT and Virtual Week performance from the alcohol use literature all found some improvement, whether it be for time- or event-based tasks, in the younger healthy controls, providing evidence that younger adults demonstrate room for improvement (Griffiths et al., 2012; Leitz et al., 2009; Paraskevaides et al., 2010; Platt et al., 2016). As mentioned earlier, perhaps our results diverged due to the addition of another experimental condition, which may have altered the overall demands of the paradigm thereby preventing improvement.

Our secondary analyses revealed both expected and unexpected findings. Contrary to predictions and previous work, none of the demographic or neuropsychological characteristics were reliably associated with the dependent variables of the Virtual Week. Behavioural and neuroimaging studies suggest that prospective memory relies on a concert of cognitive abilities including executive functions and memory (e.g., Gross et al., 2012), so a lack of association between the Virtual Week and the baseline measures was surprising. With respect to the Virtual Week in particular, Rose and colleagues (2010) found an association with working memory performance, but we found no such relationship. In addition, we had expected to see 
relationships with measures of attention and executive function (i.e., Trails A and B) and memory (i.e., paired associates and digit span), but this did not emerge in our analyses. We have no compelling explanation for this pattern of results.

A separate set of secondary analyses revealed an interesting finding regarding strategy preference. Once the Virtual Week was completed, participants were asked for their strategy preference: FT, implementation intentions, neither strategy, or both strategies. The results showed that younger adults differed from their older counterparts in their preference patterns. Whereas the most common response for younger adults $(50 \%)$ was a preference for implementation intentions, the older adults most frequently stated that they preferred neither strategy (42\%). It is unclear why there was an age discrepancy between strategy preferences. It is possible that, as mentioned earlier, the older adults found the strategies to be distracting or overly taxing when already performing a cognitively demanding task. The younger adults might have preferred the implementation intentions to FT because it is more straightforward and less effortful $^{19}$.

Collapsing across age groups, when Virtual Week performance was examined as a function of strategy preference, the results revealed an interaction between strategy preference and condition. More specifically, participants who indicated a preference for FT performed better in the FT condition compared with performance at baseline (no strategy use) and with the implementation intentions condition. Looking more closely within this analysis, there was no interaction between condition and task type (i.e., event- vs time-based tasks) suggesting that neither task types was differentially affected by the use of the FT strategy. These results suggest

\footnotetext{
${ }^{19}$ A manipulation check was not explicitly added to the paradigm but the fact that participants carried out the strategies aloud (whether it be making verbal WT statements or describing their future scenarios) provided one avenue to verify that participants were actually performing the strategies.
} 
a potential moderating effect of strategy preference on the utility of FT for prospective memory performance. It is interesting that those who preferred the implementation intentions did not significantly benefit from said strategy as compared to baseline or FT. As such, there is something unique about the preference for FT and optimized performance.

It is unclear what individual variables determine preference for the FT strategy. Age might be an important variable; indeed this group included more younger adults than older adults. Moreover, when age was entered as a covariate in these analyses, the main effect of preference and the significant Condition x Strategy interaction remained, but the follow-up tests no longer met significance. Regarding other potentially important variables, follow-up analyses examining differences in the baseline neuropsychological measures between strategy preference groups were conducted but a clear pattern failed to emerge. That is, the FT-preferred group did not stand out compared with the other strategy preference groups on any of the baseline measures. Further studies are required to uncover variables associated with a preference for particular strategies.

It is also possible that although participants were asked to select their preferred strategy, participants were actually rating which condition they perceived they had performed the best (or, if they had selected 'both' or 'neither', they thought they performed equally well/poorly across conditions). In other words, perhaps the participant ratings reflect an effect of their performance, rather than actual strategy preference. However, if this were the case, it is curious why, for those who preferred implementation intentions, they did not show significantly better performance for that condition. The lack of relationship between strategy preference and performance in the implementation intentions condition lends support to the possibility that those who prefer FT are able to utilize it effectively and that this is related to personal preference for said strategy. 
Nonetheless, this should be verified through a follow-up study whereby strategy preference is determined a priori, followed by tests of prospective memory using different strategies.

Our study paradigm was not designed to investigate how individual differences in strategy preference might relate to strategy utility with respect to prospective memory performance. As a result, we did not have an equal distribution of younger and older adults across the different strategy preference groups to validly comment on any effect of age on the relationship between strategy preference and utility. Furthermore, dividing each age group of 36 into three or four strategy preference groups would have resulted in as few as six participants in some subgroups, producing an underpowered analysis. Already when collapsed across age groups, there were unequal numbers between strategy preference groups generally, which may detract from the statistical reliability of this finding. Future studies should replicate this finding and create a study design that would identify individuals within each strategy preference group through a first phase, which would allow for recruitment of equal numbers of individuals and potentially matched on other significant characteristics.

Although our study was motivated by basic research on memory, our results may also be contextualized within the cognitive training literature. Very few memory training studies have included prospective memory despite the call for more focus on the training of tasks and processes that are directly implicated in everyday life (McDaniel \& Bugg, 2012). The small literature on prospective memory training or rehabilitation almost entirely comprises studies with clinical populations with neurological impairments rather than with older adults (for reviews see Fish et al., 2010; Hering et al., 2013; Piras et al., 2011). Thus, while our results did not support the hypotheses that we set out to test, they add to the small but growing literature concerning prospective memory training and older adults. 
Cognitive training may involve teaching specific strategies to compensate for or circumvent limitations in compromised cognitive processes, or it may involve the training of basic cognitive processes, which presumes to modify these processes per se (Hering et al., 2013; Reichman, Fiocco, \& Rose, 2010). FT and implementation intentions represent the former as they are presumed to improve performance on a specific task, as opposed to the training of basic cognitive processes (Hering et al., 2013). While imagery and visualization have a long history as a mnemonic strategy for retrospective memory in older adults (e.g., reviewed in Gross et al., 2012), evidence of transfer beyond the task for which these strategies are utilized is extremely limited, and transfer to tasks relevant to everyday living even more so (Hertzog et al., 2008). Experts have begun to stress the importance of cognitive training for transfer (e.g., McDaniel \& Bugg, 2012), and with respect to prospective memory, it has been suggested that future studies design strategies that are theoretically driven and specifically designed to train each of the four components of prospective memory (Hering et al., 2013; Kliegel, Altgassen, Hering \& Rose, 2011).

As an example, a recent study by Rose and colleagues (2015) employed a progressive training programme to improve performance on the Virtual Week task. In contrast to our work, which employed specific strategies in a single session, Rose and colleagues (2015) employed process-training whereby participants returned repeatedly for sessions of the Virtual Week, and the degree of difficulty increased adaptively as performance improved. Additional changes to the task were made with each session in order to train cue detection, monitoring, and cognitive flexibility. Finally, participants received feedback after each session. Control groups were included: one underwent sessions of music training and another was a waitlist, no-contact group. Participants in the Virtual Week-training group showed significantly greater benefits on the 
Virtual Week post-intervention as compared with the control groups. Moreover, there was evidence for both near and far transfer effects based on trends for improvement on a real-life prospective memory task (a call-back task) and on a timed task of instrumental activities of daily living, respectively. Surprisingly, there was no benefit of training on other lab-based tasks of prospective memory (e.g., the Breakfast Task) or working memory, both of which were expected. This suggests that process-based approaches may be worthwhile to pursue in that they may promote both near and far transfer, and more importantly, on ecologically valid activities. Nonetheless, the results are also sobering in that effects were not observed on direct measures of prospective memory and working memory, suggesting important room for deepening our understanding of cognitive training principles.

Taken together, prospective memory represents a relevant target for intervention due to its significance in everyday functioning, especially for older adults. Research is in the early stages, and much remains to be discovered as to which strategies are helpful, and moreover, which types of training might be most likely to generalize beyond the task in question. Processbased approaches such as that demonstrated by Rose and colleagues (2015) may be a fruitful direction for future work. In addition, studies may investigate specific strategies designed to improve the different components of prospective memory, which would ultimately benefit older adults, and which will also add to theoretical understanding of the neural and behavioural correlates of this complex cognitive ability. Finally, future work may continue to investigate cognitive strategies and more specifically, understand how individual differences explain affinity for and utility of specific strategies. 


\section{Chapter 6: General Discussion}

For decades, aging researchers studied cognition, motivation, and emotion separately but in the last several years, integrative perspectives have emerged (e.g., Carstensen, Mikels, \& Mather, 2005). The substantial body of cognitive aging literature documents reliable age-related declines in various aspects of cognitive functioning (e.g., Salthouse, 2010), but as examples of neural and cognitive plasticity are increasingly uncovered (e.g., Greenwood \& Parasuraman, 2010), attention is being paid to how contextual factors influence cognitive change throughout the lifespan (Carstensen et al., 2005). Ample evidence suggests that variables such as motivation (e.g., Hess, 2014), stereotype threat (see Lamont, Swift, \& Abrams, 2015, for a meta-analysis and review), and emotion (Carstensen et al., 2005) significantly shape the trajectory of cognitive aging. This dissertation documents two experiments embedded within this intersection between motivation and cognition, namely the cognitive ability of prospection.

Several prominent lifespan theories have speculated on how contextual factors significantly shape cognitive aging. Socioemotional selectivity theory (Carstensen, Isaacowitz, \& Charles, 1999) contends that motivational shifts occur as a result of changes in future time perspective. Younger adults typically perceive time as open ended, driving them to pursue knowledge, resources, and relationships. With aging comes an appreciation of the constraints on time, leading to a reprioritization of goals towards maintaining resources, preventing losses, and upholding emotional well-being. As relates to cognition, this theory supposes that some of the changes previously believed to be intractable may in fact reflect changes in motivation

(Carstensen et al., 2005). An offshoot of this theory is that older adults should be more motivated to invest cognitive resources in emotional tasks, and that they should engage in selective cognitive processing insofar as it is relevant for emotion regulation (Carstensen et al., 
2005). A wealth of empirical work has confirmed the existence of an age-related "positivity effect" (see Reed, Chan, \& Mikels, 2014, for a meta-analysis, although some caveats to the effect are noted; see also Grühn, Sharifian, \& Chu, 2016), or a relative age difference between younger and older adults in the processing of positive over negative information, evident in domains like attention, memory, and decision making.

Hess's (2014) selective engagement theory builds on the assertion that cognitive performance is influenced by motivational changes. It proposes that because aging is associated with disproportionately higher effort to achieve the same objective performance on cognitive tasks, older adults become increasingly selective regarding task engagement. Consequently, older adults should be less motivated to engage in demanding activities, and they should be more sensitive to self-related implications of tasks (Hess, 2014). Hess (2014) underscores that these changes may be adaptive in that they conserve limited resources, although in some contexts, a narrowing of task selection and engagement could pose problems. Recent empirical work is providing support for these claims. Smith and Hess (2015) included a physiological measure of engagement during a memory search task that included varying degrees of difficulty and a manipulation of personal relevance. Results suggested that the older adults were disproportionately sensitive to the manipulation. Moreover, the older adults showed greater reactivity to increases in task difficulty, which was interpreted to support claims of selective engagement theory.

The first part of this dissertation was inspired by these theories and their implications with respect to prospection, or future-oriented cognition. Interest in prospection has grown at an accelerating rate in the last decade (e.g., Seligman, Railton, Baumeister, \& Sripada, 2013). In 2014, Szpunar et al. integrated disparate types of prospection through a taxonomy of four modes 
- simulation, prediction, intention, and planning - further proposing that these modes vary on the degree of episodic and semantic knowledge involved. While the delineation of this taxonomy marks an important step forward in prospection research, outstanding questions have been identified, such as how might these types of prospection interact with each other, what are their functions, how do the episodic and semantic contributions matter, and how do they change across the lifespan (e.g., Michaelian et al., 2016; Seligman et al., 2013). Some have even suggested that psychology in general requires a redirection towards future-oriented cognition, and that prospection is a uniquely human ability ${ }^{20}$ that is a "central organizing feature" of attention, memory, perception, affect, and behaviour (Seligman et al., 2013).

This dissertation investigated the possibility that age-related changes in one type of prospection - simulation, or episodic future thinking - may partly reflect shifts in motivation. This dissertation was also inspired by questions from the prospection literature concerning how simulation might support two other types of prospection (intention and planning) and how this might unfold in an aging context. The first experiment tested if manipulating the self-relevance of the task might mitigate age differences typically observed in episodic future thinking. The second experiment was spurred by claims about the adaptive, motivational function of episodic future thinking per se. Experts have questioned why it is that humans can engage in episodic thinking - past or future - altogether. Some have claimed that episodic future thinking is beneficial for processes such as emotion regulation, decision making, problem solving, prospective memory, and goal achievement (e.g., Jing, Madore, \& Schacter, 2016; Schacter,

\footnotetext{
${ }^{20}$ However, the assertion that prospection is uniquely human is contested in the literature, with some researchers maintaining that animals are unable to plan or act based on anticipated motivational states not currently experienced (see the Bischof-Kohler Hypothesis, described in Suddendorf \& Corballis, 1997), while others have found results interpreted as evidence of prospection in animals (e.g., Osvath \& Osvath, 2008 for evidence in apes and Correia, Dickinso, \& Clayton, 2007, for evidence in scrub jays). This remains an ongoing debate (e.g., Martin-Ordas, Atance, \& Louw, 2012).
} 
2012; Seligman et al., 2013; Suddendorf \& Corballis, 2007; Taylor et al., 1999), and that perhaps humans have developed the capacity for episodic thinking precisely to permit the accomplishment of such future-oriented goals (Taylor et al., 1999; Taylor \& Schneider, 1989). Conway and colleagues (2016) assert that the remembering-imagining system (RIS) is a central component of one's goal system (Conway \& Pleydell-Pearce, 2000). The RIS is conceptualized as a bellcurve, with the apex being the 'now,' or present, and the tails representing the past and the future, respectively. Movement towards the tails is associated with decreased access and reduced episodic specificity, but increases in "the schematic/generic/stereotypical, culturally specified" (Conway et al., 2016). They maintain that "raised access" to recent memories helps one to ascertain progress towards goals, which, in turn, informs selection of future activities.

\section{Summary and Implications of Experiment 1}

The results from the first experiment showed that prompting younger and older adults with age-appropriate goals did not influence degree of episodic detail in future simulations, which was contrary to the primary predictions. However, goals differentially affected select phenomenological characteristics. Younger adults appeared to be more sensitive to this effect; the older adults' phenomenological ratings showed less differentiation as a function of the different goals. Overall, the results suggest that eliciting age-appropriate motivational constructs is insufficiently powerful to offset the semanticization of future simulations associated with aging. Nonetheless, given the influence on the phenomenological features, the results also suggest that future simulation is not insensitive to motivation. Future research should employ alternative paradigms to further explore how personal goals shape future simulation; this is especially important given the predominance of theories predicting a relationship. For example, researchers might utilize more personal and elaborated cues (e.g., Kwan et al., 2015) as these 
have been shown to modulate episodic detail in populations that typically demonstrate deficits in this domain.

More broadly, characterizing the course of prospection in healthy aging is important insofar as it can inform taxonomic theories (Szpunar et al., 2014), as well as theories about cognitive aging more generally. It also informs knowledge about pathological aging. Future thinking deficits are beginning to be delineated across the dementia subtypes (for a review, see Irish \& Piolino, 2016). For example, individuals with Alzheimer's disease demonstrate symmetrical reductions in episodic details making up their autobiographical memories and future thinking scenarios, as compared with healthy controls, suggesting an important role for medial temporal and posterior parietal regions since these are implicated in this disease (Addis, Sacchetti, Ally, Budson, \& Schacter, 2009; Irish \& Piolino, 2016). Individuals with semantic dementia, who typically show relatively intact episodic memory as compared with Alzheimer's disease but pronounced semantic deficits, show asymmetrical deficits: autobiographical memory is preserved while future thinking - and in particular, episodic detail - is compromised (Duval et al., 2012; Irish et al., 2012; Irish \& Piolino, 2016). Irish and Piolino (2016) note that it is not yet possible to use future thinking to resolve differential diagnoses between dementias, but with more fine-grained measures, it may eventually become another helpful tool in neuropsychological batteries (Ward, 2016). The authors also state that the emphasis on episodic memory problems as central to functional impairment is important, but broadening the focus to include how compromised future thinking impacts daily living warrants attention since it likely influences prospective memory, decision making, and sense of self (Irish \& Piolino, 2016). Indeed, in order for future thinking to represent a relevant neuropsychological assessment target, it should specifically inform diagnosis and intervention (Lezak et al., 2012), and for this to be the 
case, future thinking must show discriminant validity between issues of interest and significance for adaptive functioning (Ward, 2016). This remains an important direction for future research. Miloyan and colleagues (2016) discuss how future thinking may also be of importance with respect to anxiety and depression in aging populations ${ }^{21}$ but its role remains to be elucidated. In point of fact, anxiety and mood disorders represent one of the most common mental health problems in older adults (Miloyan, Pachana, \& Suddendorf, 2016; Reynolds, Pietrzak, El-Gabalawy, Mackenzie, \& Sareen, 2015). These disorders are associated with cognitive impairment in older adults, and appropriate treatment can also improve cognition (Miloyan et al., 2016). Future thinking is already established as an important target in anxiety and depression in younger populations. Anxiety is characterized by attentional biases towards threatening stimuli and future-oriented worries, while depression is associated with decreased positive and increased negative content of past and future thoughts and beliefs about the self, others, and the world (Beck, 1976). This is in contrast to the nature of everyday future thinking, which, for the average, euthymic individual, tends to comprise more positive than negative future thoughts (e.g., Barsics, van der Linden, \& D’Argembeau, 2016; D'Argembeau et al., 2011).

Evidence from intervention studies with younger adults supports a promising role for imagery-based interventions. For example, McEvoy et al. (2015) have found large effect sizes for imagery-enhanced CBT interventions for social anxiety. Patients are taught to attend to and modify past, present and future negative episodic, imagined scenarios. Other interventions (e.g., Raes, Williams, \& Hermans, 2009; Williams, Teasdale, Segal, \& Soulsby, 2000) are focused on

\footnotetext{
${ }^{21}$ The younger adults in our experiments showed significantly higher scores than older adults on the DASS-21, which is typical in cognitive aging research. Indeed, the older adults in both experiments reported quite low scores on the DASS-21. However, the DASS-21 was not significantly related to any of the main outcome measures in our experiments. Nonetheless, the studies cited in this section suggest that anxiety and depression are concerns for older adults in the general population. Future studies may look to include more representative samples or select specifically for older adults who report higher levels of depression and anxiety in order to begin answering questions about how future thinking, memory, and other important variables are implicated in this age group.
} 
improving specificity in episodic thinking, which is associated with decreased depressive symptoms, since overgeneral autobiographical memory and future thinking is a key cognitive marker of depression (e.g., Addis, Hach, \& Tippett, 2016; Williams et al., 2006). The results from those studies are promising and suggest a potential relationship between episodic specificity and well-being. Relatedly, Madore and colleagues (2014) showed that a specificity induction was successful in increasing episodic details for past and future simulations, and importantly, both older and younger adults benefited. Finally, Jing et al. (2016) demonstrated that using a specificity induction to increase degree of episodic detail in future simulations of worrisome events increased positive affect and decreased negative affect. It was also associated with improved performance on a means-end problem solving task and improved self-efficacy with respect to coping with the worrisome event. Given this promising work with younger adults and related work with older adults, Miloyan et al.'s (2016) claim that future-oriented cognitions play an important but under investigated role in anxiety and mood conditions in older adults represents a significant direction for future research and intervention.

\section{Summary and Implications of Experiment 2}

The results from the second experiment were surprising insofar as neither future thinking nor implementation intentions improved prospective memory performance. The fact that implementation intentions were ineffective was especially remarkable given the body of work suggesting its utility (for a review, see Chen et al., 2015). That future thinking was unhelpful was less striking given the dearth of studies employing the strategy in older adults (e.g., Altgassen et al., 2015). Nonetheless, a small but notable amount of work from the alcohol literature (e.g., Griffiths et al., 2012; Leitz et al., 2009; Paraskevaides et al., 2010; Platt et al., 2016) and the TBI literature (e.g., Mioni et al., 2017) have found that future thinking 
significantly improves prospective memory performance on the Virtual Week in particular. We can only speculate as to why no positive relationships emerged. With respect to the motivating question for the experiment, however (i.e., that a primary function of future thinking is to facilitate future action completion, such as with prospective memory), a clear answer failed to emerge.

Nonetheless, the results are equivocal, because they also suggested that when individual differences in strategy preference are accounted for, future thinking may indeed improve prospective memory performance. More specifically, those who indicated a preference for future thinking showed a benefit in prospective memory performance as a function of that strategy. This dovetails with increasing trends towards incorporating individual differences in the field of memory and prospection. For example, Sheldon, Farb, Palombo, and Levine (2016) found evidence for individual differences in trait-like tendencies towards episodic versus semantic autobiographical recollections in younger adults, as measured through a validated self-report measure (the Survey of Autobiographical Memory, SAM; Palombo, Williams, Abdi, \& Levine, 2013) about their mnemonic experience. Moreover, the two patterns (greater episodic tendencies versus greater semantic tendencies) were each associated with distinct MTL connectivity signatures. Those individuals naturally tending towards highly episodic recollections showed MTL connectivity with posterior regions, whereas those tending towards semantic recollections displayed MTL connectivity with inferior and middle PFC regions. The authors explain that, consistent with models of autobiographical memory (Conway \& Pleydell-Pearce, 2000), memories can be retrieved beginning at the conceptual, more abstract, semantic tier, after which details are filled in, but they can also be generated more directly at the episodic detail level, bypassing the conceptual tier. They encourage researchers to incorporate self-report measures 
such as theirs to account for individual differences in their work. Our results align with this request; indeed one future direction would be to directly test how individual differences in preferences for future thinking might predict the utility of this strategy on prospective memory performance, perhaps employing a measure like the SAM (Palombo et al., 2013).

\section{Limitations}

Limitations and caveats have been described in the specific discussion sections for each experiment but will be briefly reviewed here. Regarding the first experiment, the main limitations were failing to include neutral cue words, similar to those utilized in other studies of future thinking. This would have facilitated comparisons across studies, and would have also confirmed if any of the differences observed with the phenomenological ratings were indeed related to the motivational salience of the cues, rather than to lexical properties or other features of the cue words. Additional limitations include not having autobiographical memory conditions, which were left out due to time constraints dictated by the study parameters. Finally, not validating the differential importance of the cue words in the current sample introduced ambiguity in drawing definitive conclusions about the role of goal activation. A tighter study design would have validated the cue words derived from Lapp and Spaniol (2016) in the current sample prior to conducting the main paradigm. Although we did our best to address this shortcoming through an additional survey, this solution was less than ideal since only the original sample of older adults could be re-contacted, and it was almost one year after testing. Nevertheless, we were grateful that a manuscript reviewer noted this weakness such that we were able to address it to some degree, hopefully strengthening the integrity of the study design.

As regards the second study, the data might have been more stable if participants performed more than one day of the Virtual Week for each condition. Again, due to time 
constraints, adding days was not an option. Alternatives might have been to employ a betweensubjects design such that three different groups were allotted to each of the conditions (i.e. no strategy, future thinking, and implementation intentions) so that each condition would have comprised more virtual days. However, the younger sample would have had to be dropped from the design due to the infeasibility of running six different experimental groups. Conversely, more time might have been spent training or calibrating the task for the older adults since it is possible that adding the strategies exceeded cognitive resources; indeed the older adults might have already been overloaded with the baseline demands of the Virtual Week.

Given the null findings, however, future studies may wish to explore one of these alternative designs. We still believe it is important to include active control conditions in order to substantiate strong claims about the utility of a particular cognitive strategy; indeed all of the other prospective memory studies finding support for future thinking have neglected to include a comparison strategy, which represents a significant limitation for clear conclusions.

A final point of consideration, relevant to both studies, concerns the participant characteristics. Across both studies, participants were largely female, and the younger adults were all university students. It would be interesting to explore if different ratios of genders or a selection of younger adults beyond the university setting might impact the results. Moreover, exploring the role of other features such as socio-economic status or culture also warrants further study. Speculating how participant characteristics might impact the results seems clearer for the first experiment, given the importance of motivational factors in that study. Including younger adults from outside the university setting may reduce this age group's sensitivity to the achievement cues for the phenomenological ratings, since university students are embedded within an academic setting that highly reinforces achievement values. The university population 
is quite diverse in terms of culture and class, but unfortunately we did not collect this demographic information and thus, we were unable to investigate how these variables might relate to the goal domains and future-thinking scenarios generated in the first experiment. It is possible that the importance of the goal domains might have been ranked differently (or new categories generated altogether) if factors such as culture or class were accounted for. These represent exciting avenues for future studies.

\section{Final remarks}

Despite the fact that many of the primary predictions of both experiments were unsupported, secondary outcomes emerged, which suggest interesting possibilities for future research. The results add to the nascent interest in prospection within cognitive aging as well as within psychology at large (e.g., Seligman et al., 2013). It is an exciting period: Cole (2016) noted that 2016 witnessed the publication of two special issues on future-oriented cognition, in addition to several books (e.g., Michaelian, Klein, \& Szpunar, 2016; Seligman et al., 2016) synthesizing the literature and presenting lively theoretical debates. This dissertation may be contextualized within this growing body of work, which will only continue to reveal and refine our understanding of prospection and motivation across the lifespan. 


\section{Tables}

Table 1: Demographic and Neuropsychological Data (Experiment 1)

\begin{tabular}{|c|c|c|c|c|}
\hline & $\begin{array}{l}\text { Younger } \\
(\mathrm{N}=33)\end{array}$ & $\begin{array}{l}\text { Older } \\
(\mathrm{N}=34)\end{array}$ & & \\
\hline \multicolumn{5}{|l|}{ Demographics } \\
\hline Age & $20.91(3.47)$ & $72.06(5.08)$ & & \\
\hline Female & 28 & 30 & & \\
\hline Male & 5 & 4 & & \\
\hline Cognitive Measures & & & $t$-test & $p$ value \\
\hline Education & $14.42(2.66)$ & $15.76(2.48)$ & $t(65)=-2.14$ & $<.05$ \\
\hline Shipley & $29.06(4.44)$ & $35.91(3.40)$ & $t(65)=-7.10$ & $<.001$ \\
\hline DSC & $82.64(10.74)$ & $58.12(10.52)$ & $t(65)=9.44$ & $<.001$ \\
\hline MMSE & N/A & $29.00(1.18)$ & & \\
\hline \multicolumn{5}{|l|}{ Affective Measures } \\
\hline Depression & $7.03(6.46)$ & $4.41(4.14)$ & $t(65)=1.98$ & $n s$ \\
\hline Anxiety & $7.58(8.84)$ & $2.65(2.19)$ & $t(65)=3.15$ & $<.01$ \\
\hline Stress & $12.00(7.11)$ & $1.12(.48)$ & $t(65)=3.62$ & .001 \\
\hline \multicolumn{5}{|l|}{ PANAS } \\
\hline Pos-Pre & $27.18(6.46)$ & $35.76(8.31)$ & $t(65)=-4.71$ & $<.001$ \\
\hline Pos-Post & $28.85(9.35)$ & $34.00(9.21)$ & $t(65)=-2.27$ & $<.05$ \\
\hline Neg-Pre & $12.79(3.28)$ & $11.47(2.09)$ & $t(65)=1.97$ & $n s$ \\
\hline Neg-Post & $12.91(3.26)$ & $11.91(3.90)$ & $t(65)=1.13$ & $n s$ \\
\hline
\end{tabular}

Note. Mean (Standard deviations are given in parentheses)

DSC $=$ Digit-Symbol Coding; MMSE $=$ Mini-Mental State Examination $;$ Pos-Pre $=$ Positive PANAS scale prior to the main task; Pos-Post $=$ Positive PANAS scale after the main task; NegPre - Negative PANAS scale, prior to the main task; Neg-Post - Negative PANAS scale, after the main task; $\mathrm{ns}=$ non significant 
Table 2: Demographic and Neuropsychological Data (Experiment 2)

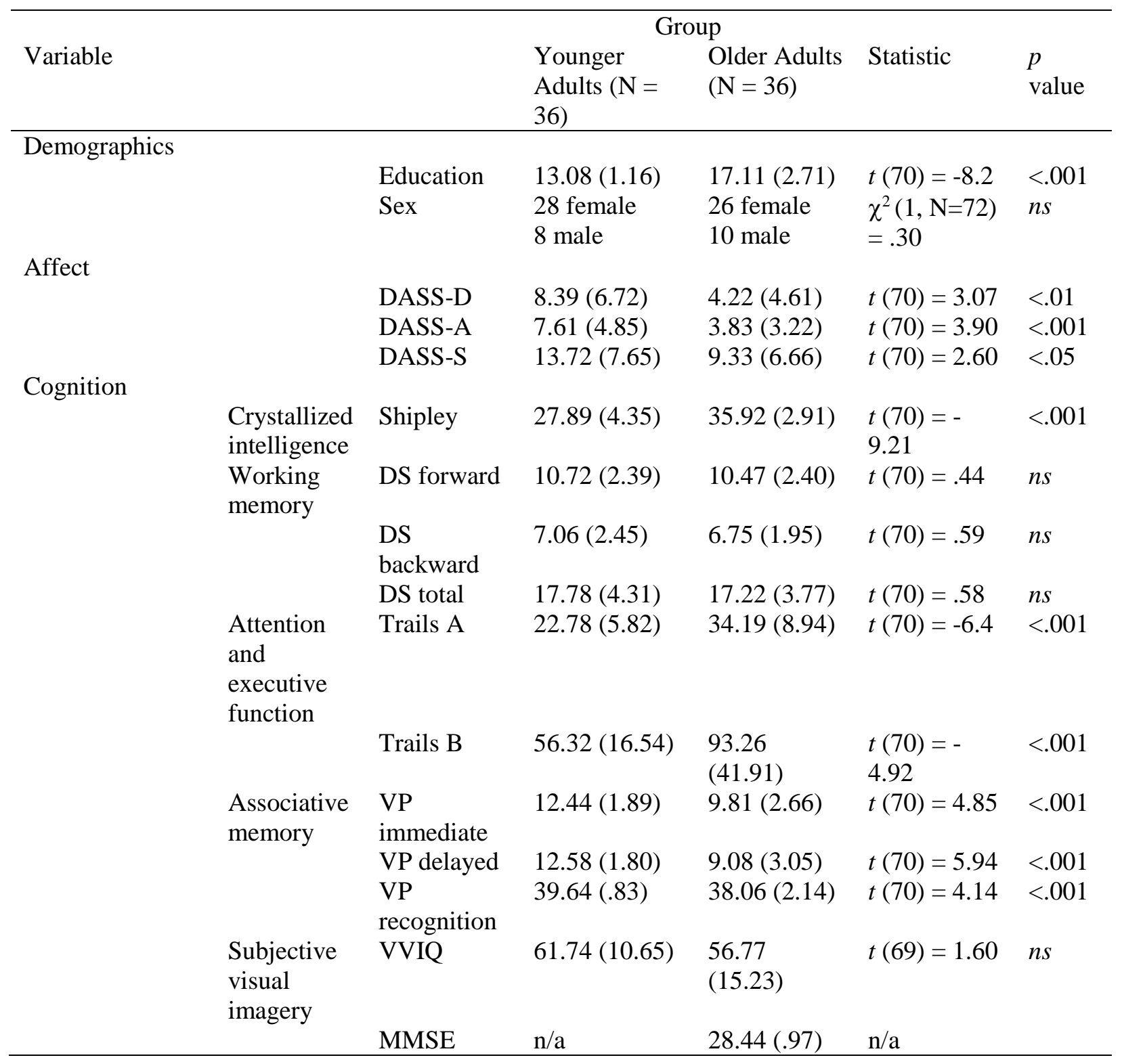

Note. Mean (Standard Deviations are given in parentheses)

DASS $=$ Depression Anxiety Stress Scale; DS = Digit Span; VP = Verbal Paired Associates;

VVIQ = Vividness of Visual Imagery Questionnaire; MMSE = Mini-Mental Status Examination; ns $=$ non-significant 
Table 3: Virtual Week Performance

\begin{tabular}{|c|c|c|c|c|c|c|c|c|c|c|c|c|}
\hline & \multicolumn{12}{|c|}{ Group } \\
\hline \multirow[t]{2}{*}{$\begin{array}{l}\text { Conditio } \\
\mathrm{n}\end{array}$} & \multicolumn{6}{|c|}{$\begin{array}{l}\text { Younger } \\
(\mathrm{N}=36)\end{array}$} & \multicolumn{6}{|c|}{$\begin{array}{c}\text { Older } \\
(\mathrm{N}=36)\end{array}$} \\
\hline & $\begin{array}{l}\text { Correc } \\
\mathrm{t}\end{array}$ & $\begin{array}{l}\text { Mis } \\
\mathrm{s}\end{array}$ & $\begin{array}{l}\text { Littl } \\
\text { e late }\end{array}$ & $\begin{array}{l}\text { Lot } \\
\text { late }\end{array}$ & $\begin{array}{l}\text { Littl } \\
\text { e } \\
\text { early }\end{array}$ & $\begin{array}{l}\text { Lot } \\
\text { earl } \\
\mathrm{y}\end{array}$ & $\begin{array}{l}\text { Correc } \\
\mathrm{t}\end{array}$ & $\begin{array}{l}\text { Mis } \\
\mathrm{s}\end{array}$ & $\begin{array}{l}\text { Littl } \\
\text { e late }\end{array}$ & $\begin{array}{l}\text { Lot } \\
\text { late }\end{array}$ & $\begin{array}{l}\text { Littl } \\
\text { e } \\
\text { early }\end{array}$ & $\begin{array}{l}\text { Lot } \\
\text { earl } \\
\mathrm{y}\end{array}$ \\
\hline \multicolumn{13}{|l|}{ Baseline } \\
\hline $\begin{array}{l}\text { Regular } \\
\text { event }\end{array}$ & 0.69 & 0.08 & 0.07 & $\begin{array}{r}0.1 \\
5\end{array}$ & 0.00 & 0.00 & 0.58 & 0.14 & 0.11 & $\begin{array}{r}0.1 \\
4\end{array}$ & 0.03 & 0.00 \\
\hline Regular & & & & 0.0 & & & & & & 0.1 & & \\
\hline $\begin{array}{l}\text { time } \\
\text { Irregular }\end{array}$ & 0.57 & 0.24 & 0.06 & $\begin{array}{r}0 \\
0.0\end{array}$ & 0.01 & 0.10 & 0.56 & 0.14 & 0.10 & $\begin{array}{r}1 \\
0.0\end{array}$ & 0.06 & 0.04 \\
\hline $\begin{array}{l}\text { event } \\
\text { Irregular }\end{array}$ & 0.68 & 0.19 & 0.00 & $\begin{array}{r}6 \\
0.1\end{array}$ & 0.00 & 0.06 & 0.56 & 0.31 & 0.03 & $\begin{array}{r}7 \\
0.1\end{array}$ & 0.03 & 0.01 \\
\hline time & 0.44 & 0.24 & 0.11 & 8 & 0.00 & 0.01 & 0.36 & 0.36 & 0.07 & 8 & 0.03 & 0.00 \\
\hline \multicolumn{13}{|l|}{ FT } \\
\hline Regular & & & & 0.0 & & & & & & 0.0 & & \\
\hline event & 0.88 & 0.04 & 0.06 & 3 & 0.00 & 0.00 & 0.71 & 0.14 & 0.08 & 4 & 0.03 & 0.00 \\
\hline Regular & & & & 0.0 & & & & & & 0.0 & & \\
\hline time & 0.71 & 0.10 & 0.13 & 1 & 0.03 & 0.04 & 0.67 & 0.13 & 0.06 & 7 & 0.06 & 0.03 \\
\hline Irregular & & & & 0.0 & & & & & & 0.1 & & \\
\hline event & 0.81 & 0.13 & 0.01 & 3 & 0.00 & 0.01 & 0.54 & 0.24 & 0.01 & 3 & 0.00 & 0.08 \\
\hline Irregular & & & & 0.1 & & & & & & 0.1 & & \\
\hline time & 0.57 & 0.16 & 0.11 & 0 & 0.00 & 0.04 & 0.38 & 0.31 & 0.17 & 4 & 0.01 & 0.00 \\
\hline \multicolumn{13}{|l|}{ WT } \\
\hline Regular & & & & 0.0 & & & & & & 0.0 & & \\
\hline event & 0.89 & 0.03 & 0.04 & 4 & 0.00 & 0.00 & 0.71 & 0.08 & 0.10 & 7 & 0.04 & 0.00 \\
\hline Regular & & & & 0.0 & & & & & & 0.0 & & 006 \\
\hline $\begin{array}{l}\text { time } \\
\text { Irregular }\end{array}$ & 0.69 & 0 & 0.15 & $\begin{array}{r}1 \\
0.0\end{array}$ & 1 & 4 & 7 & 4 & 0.13 & $\begin{array}{r}8 \\
0.0\end{array}$ & 03 & 0.06 \\
\hline event & 0.76 & 0.19 & 0.00 & 3 & 0.00 & 0.01 & 0.60 & 0.36 & 0.00 & 1 & 0.00 & 0.03 \\
\hline Irregular & & & & 0.1 & & & & & & 0.2 & & \\
\hline time & 0.47 & 0.33 & 0.00 & 5 & 0.00 & 0.03 & 0.29 & 0.29 & 0.04 & 2 & 0.06 & 0.10 \\
\hline
\end{tabular}


Table 4: Distribution of Strategy Preference between Age Groups

\begin{tabular}{llll}
\hline & \multicolumn{2}{c}{ Group } \\
Strategy Preference & Younger adults & Older adults & Total N \\
\hline FT & $30.6 \%(11)$ & $22.2 \%(8)$ & 19 \\
WT & $50.0 \%(18)$ & $19.4 \%(7)$ & 25 \\
Neither & $19.4 \%(7)$ & $41.7 \%(15)$ & 22 \\
Both & $0 \%$ & $16.7 \%(6)$ & 6 \\
\hline
\end{tabular}




\section{Figures}

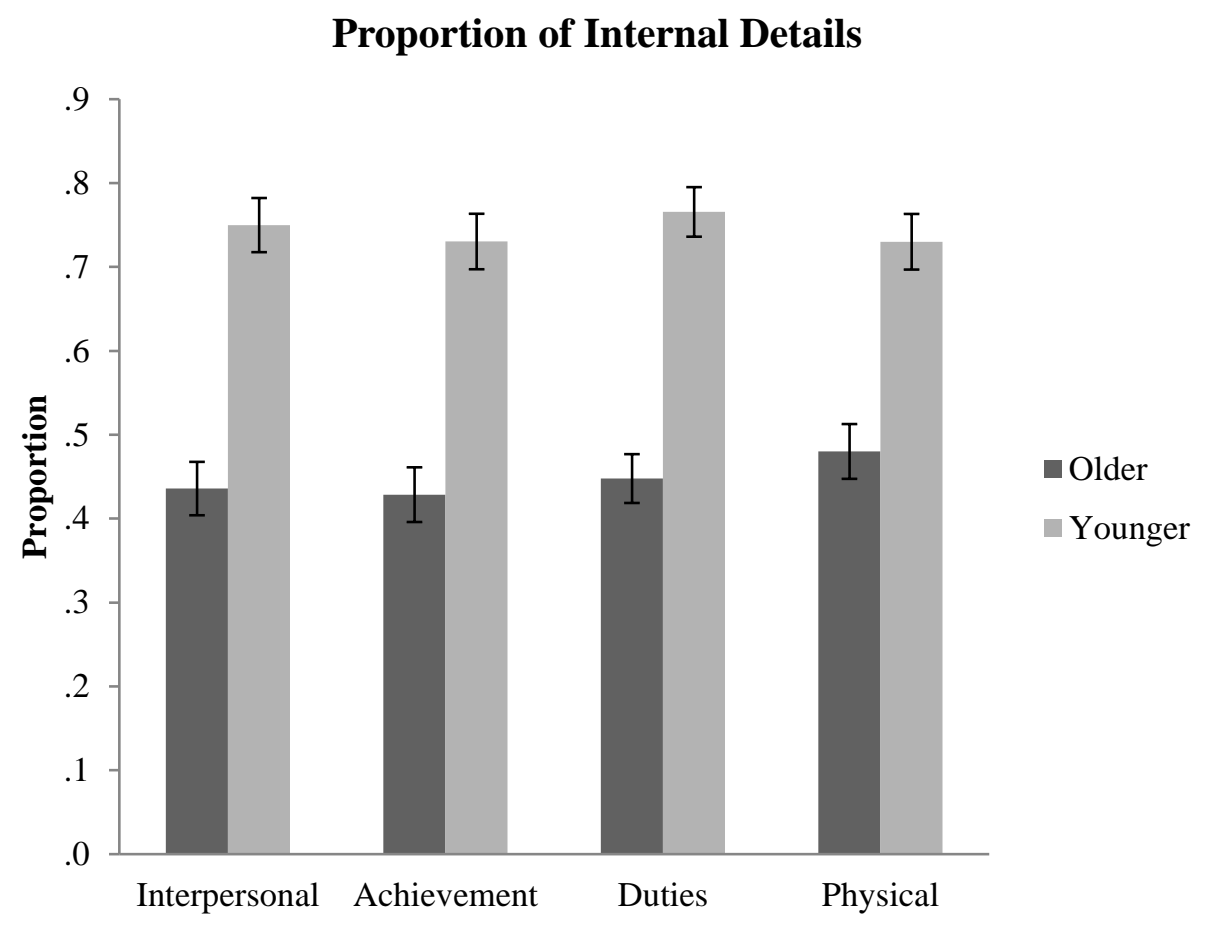

Figure 1. FTI Scoring: Proportion of Internal Details.

Mean proportion of internal to overall details for each cue type. Standard errors are represented by the error bars attached to each column. 


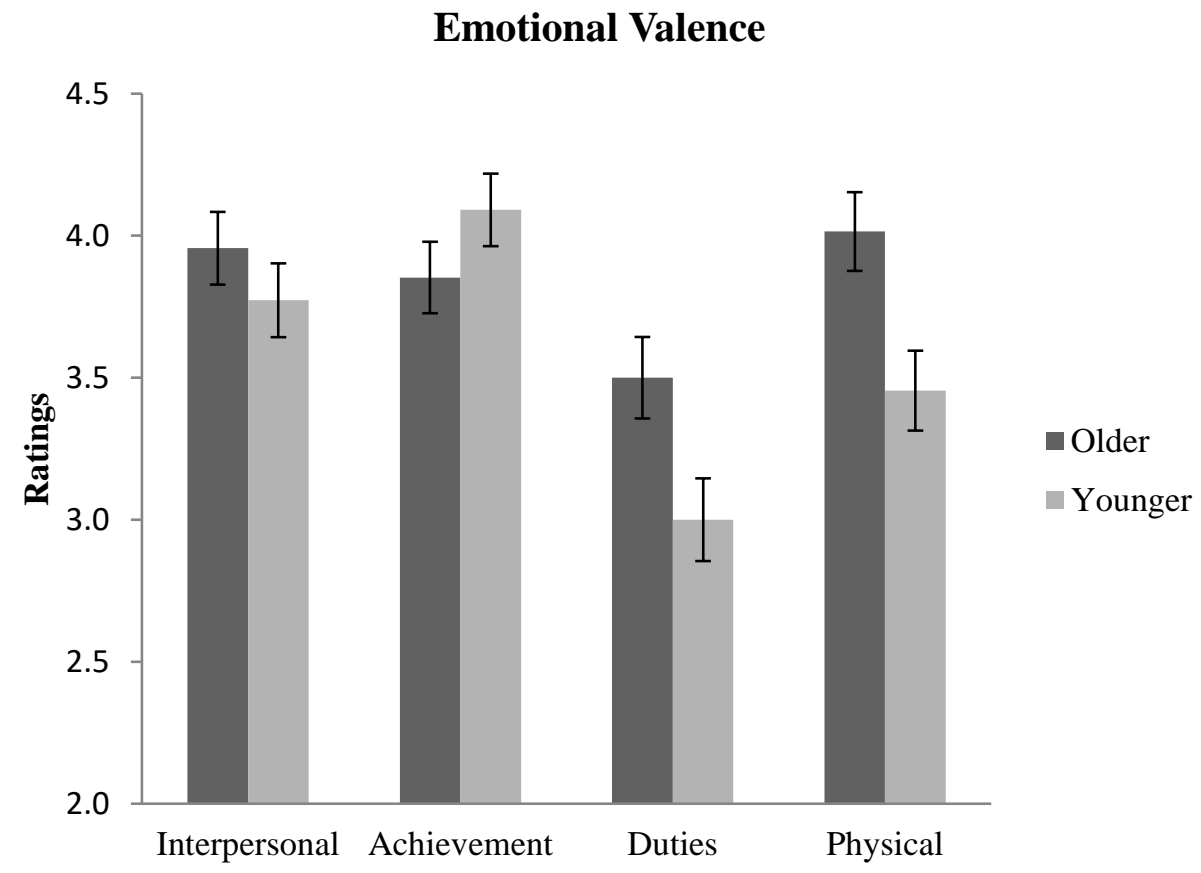

Figure 2. Phenomenological Ratings: Emotional Valence

Mean ratings of emotional valence for each cue type. Ratings correspond to $1=$ very negative, to $5=$ very positive. Standard errors are represented by the error bars attached to each column. 


\section{Emotional Intensity}

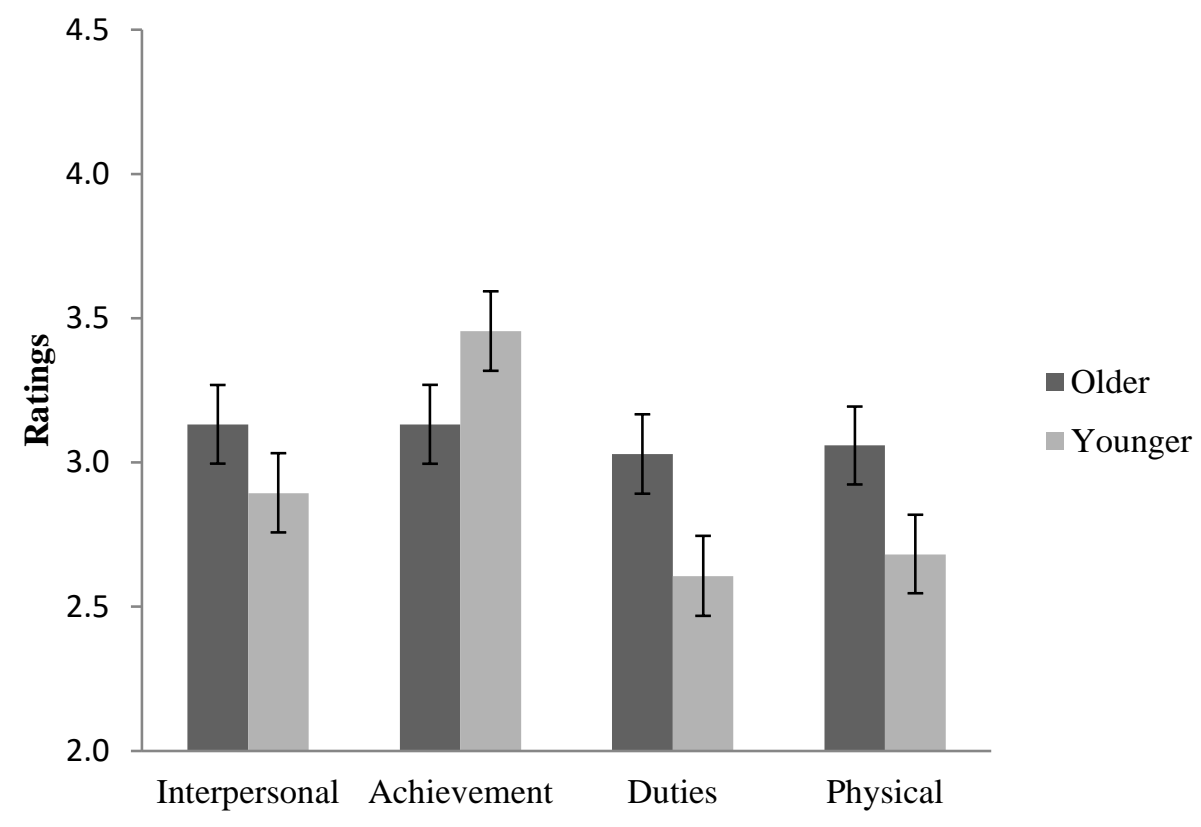

Figure 3. Phenomenological Ratings: Emotional Intensity

Mean ratings of emotional intensity for each cue type. Ratings range from $1=$ non emotional, to $5=$ highly emotional. Standard errors are represented by the error bars attached to each column. 


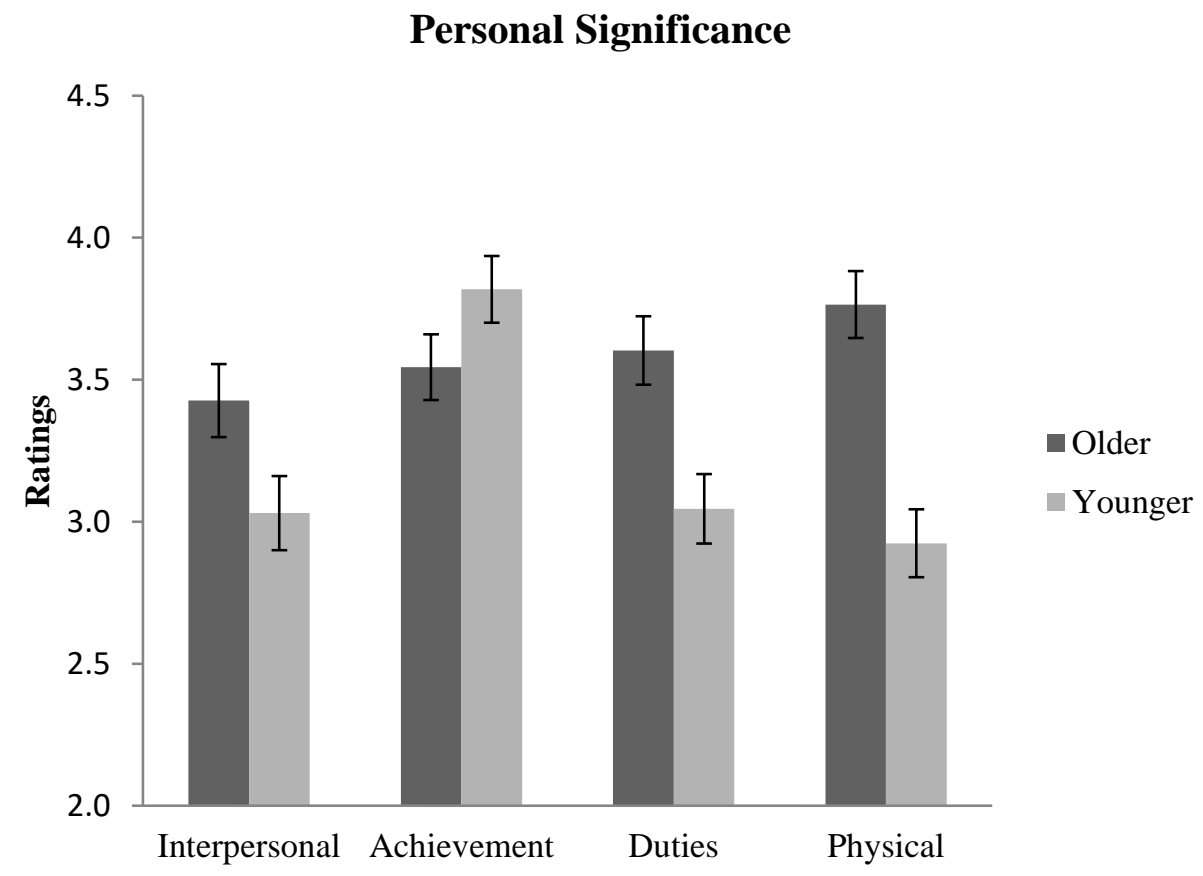

Figure 4. Phenomenological Ratings: Personal Significance

Mean ratings for personal significance ratings for each cue type. Ratings correspond to $1=$ insignificant, to $5=$ life changing. Standard errors are represented by the error bars attached to each column. 


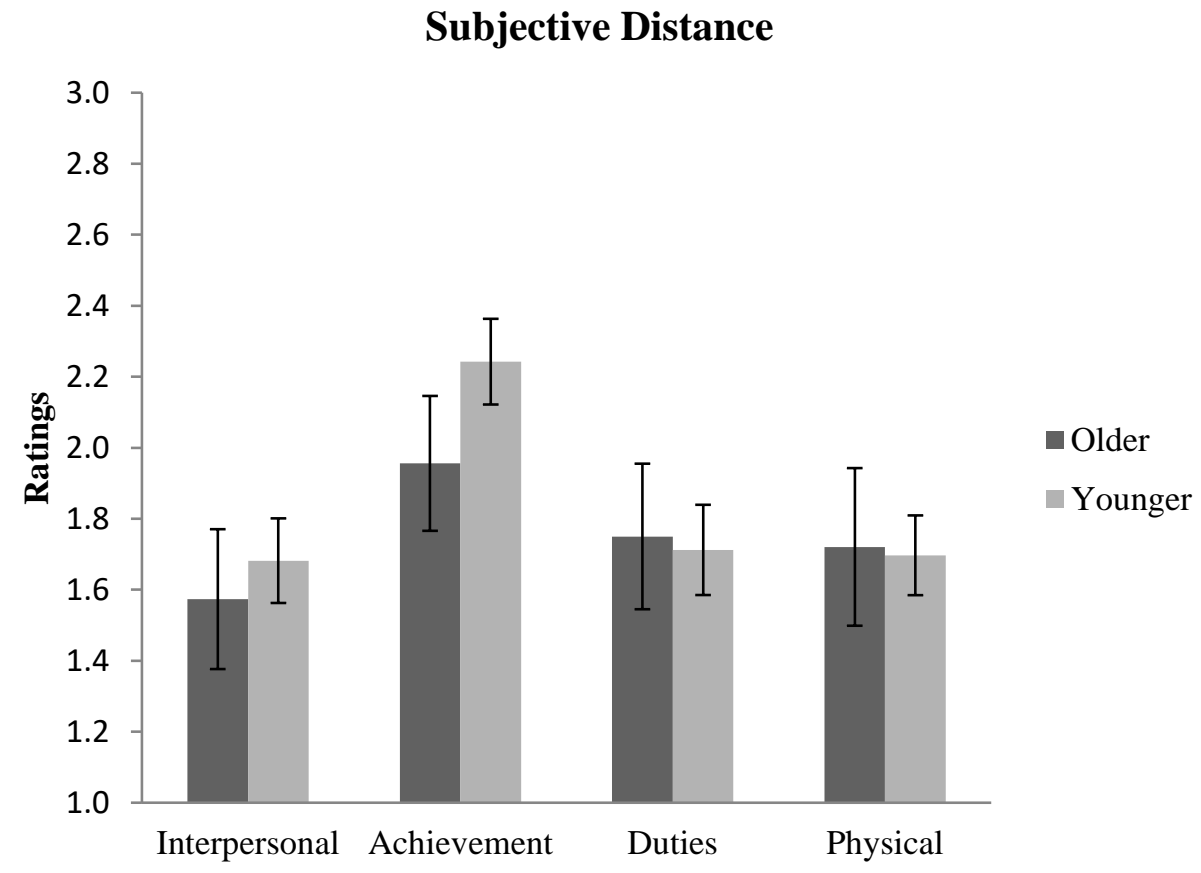

Figure 5. Phenomenological Ratings: Subjective Distance

Mean ratings for projected date of future simulation for each cue type. Ratings correspond to $1=$ next few weeks, $2=$ next few months, and $3=$ next few years. Standard errors are represented by the error bars attached to each column. 


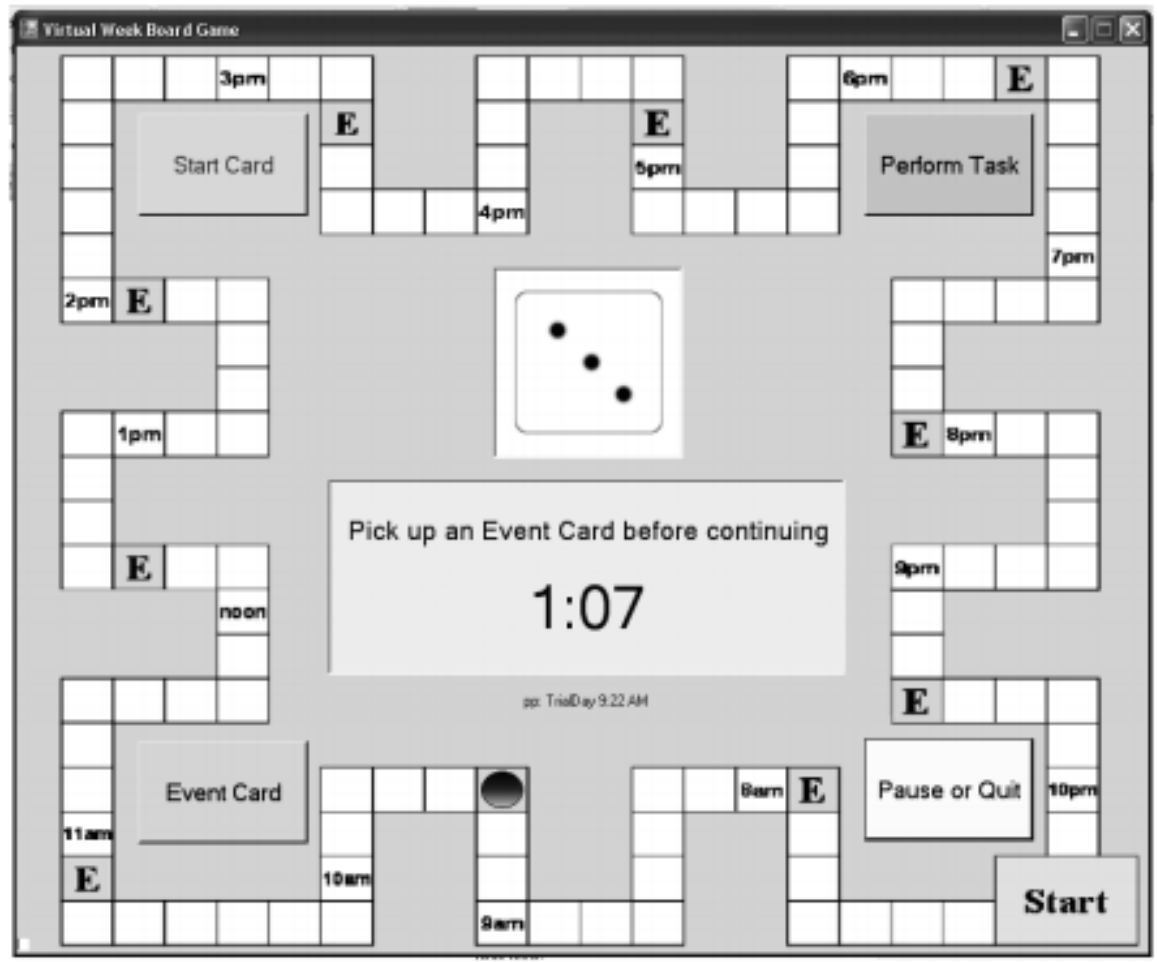

Figure 6. Snapshot of the Virtual Week Board Game 


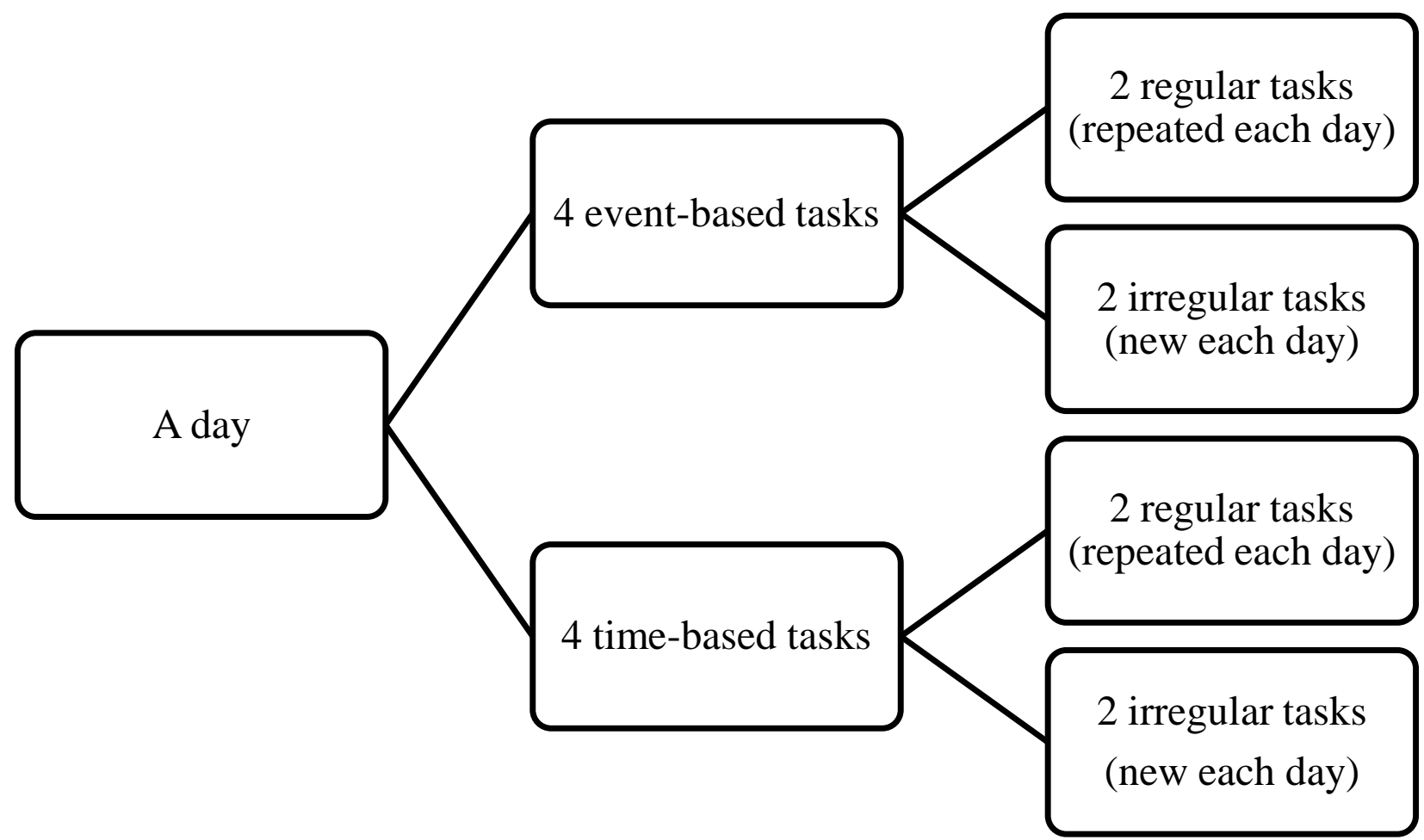

Figure 7. Depiction of Tasks within a "day" of the Virtual Week 


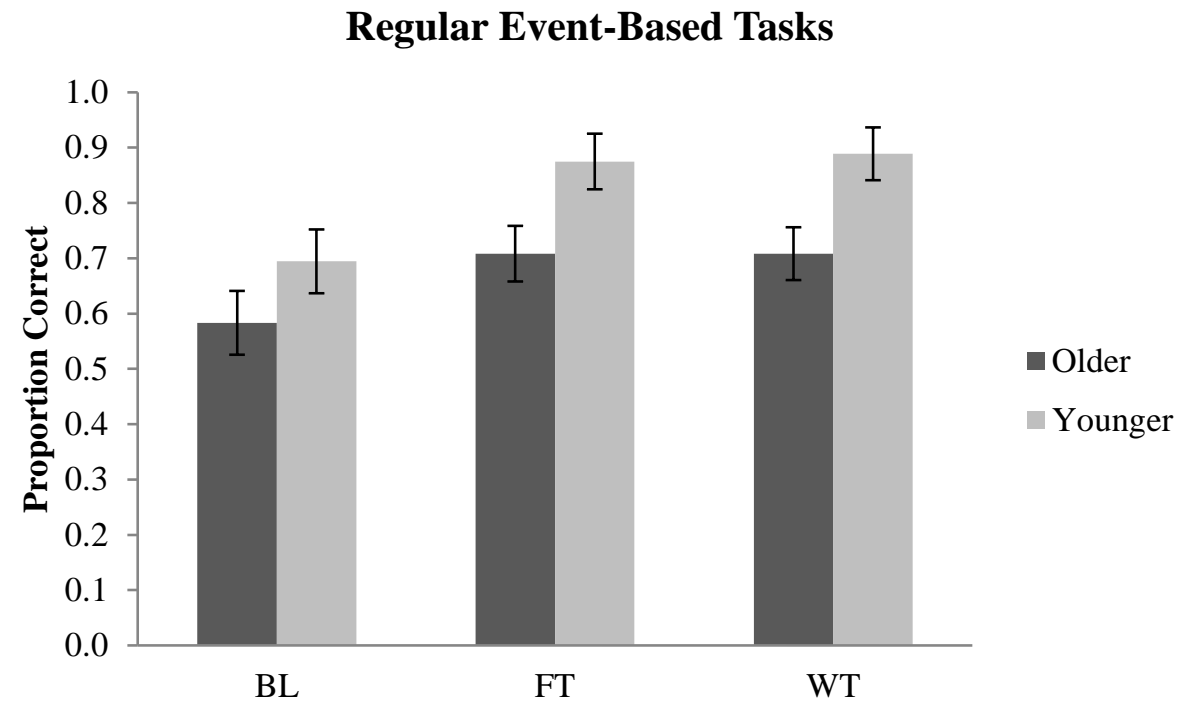

Figure 8. Regular Event-Based Tasks

Mean proportion correct for each condition (BL = baseline; FT = future thinking; WT = whenthen, or implementation intentions). Standard errors are represented by the error bars attached to each column. 


\section{Regular Time-Based Tasks}

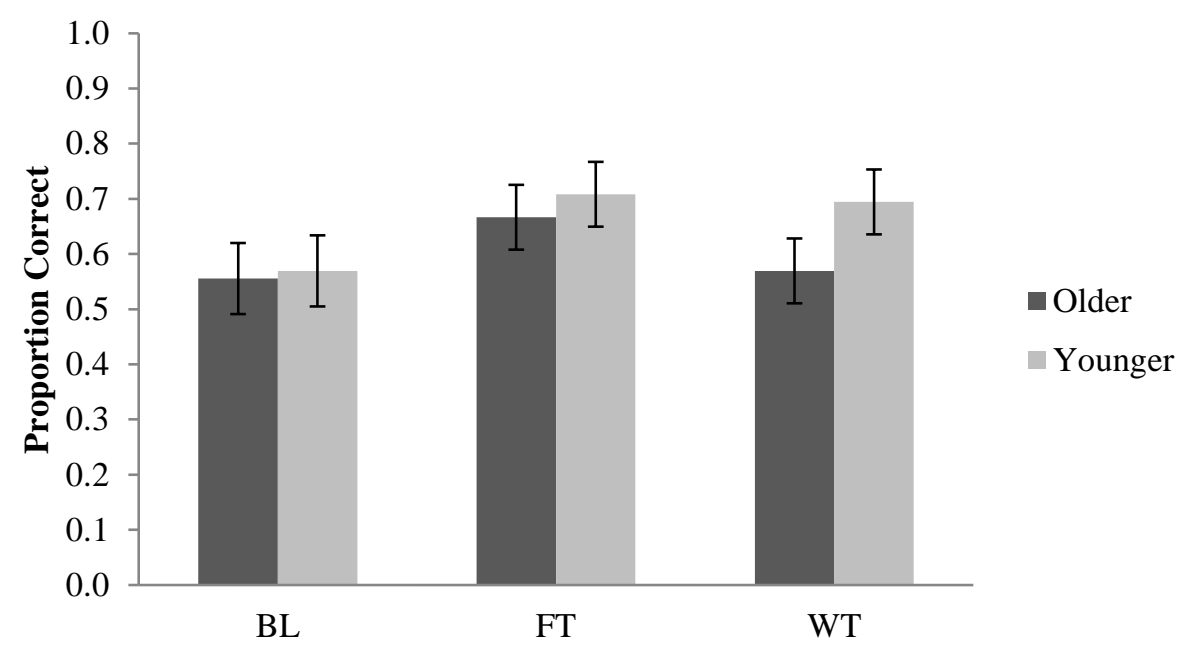

Figure 9. Regular Time-Based Tasks

Mean proportion correct for each condition (BL = baseline; FT = future thinking; WT = whenthen, or implementation intentions). Standard errors are represented by the error bars attached to each column. 


\section{Irregular Event-Based Tasks}

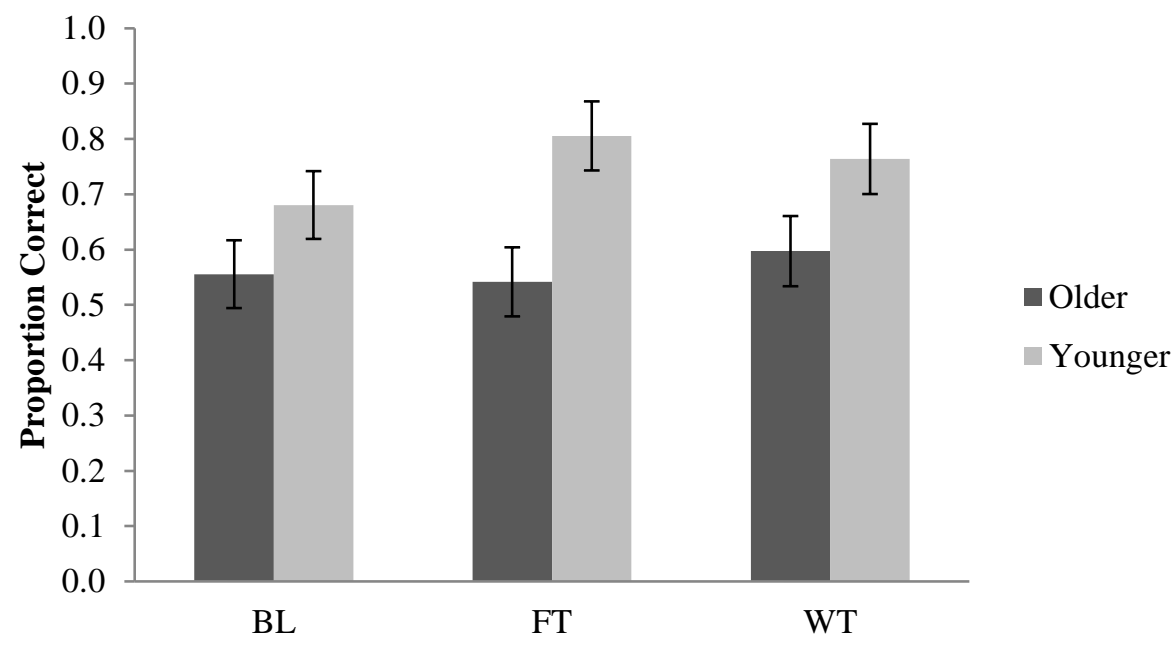

Figure 10. Irregular Event-Based Tasks

Mean proportion correct for each condition (BL = baseline; FT = future thinking; WT = whenthen, or implementation intentions). Standard errors are represented by the error bars attached to each column. 


\section{Irregular Time-Based Tasks}

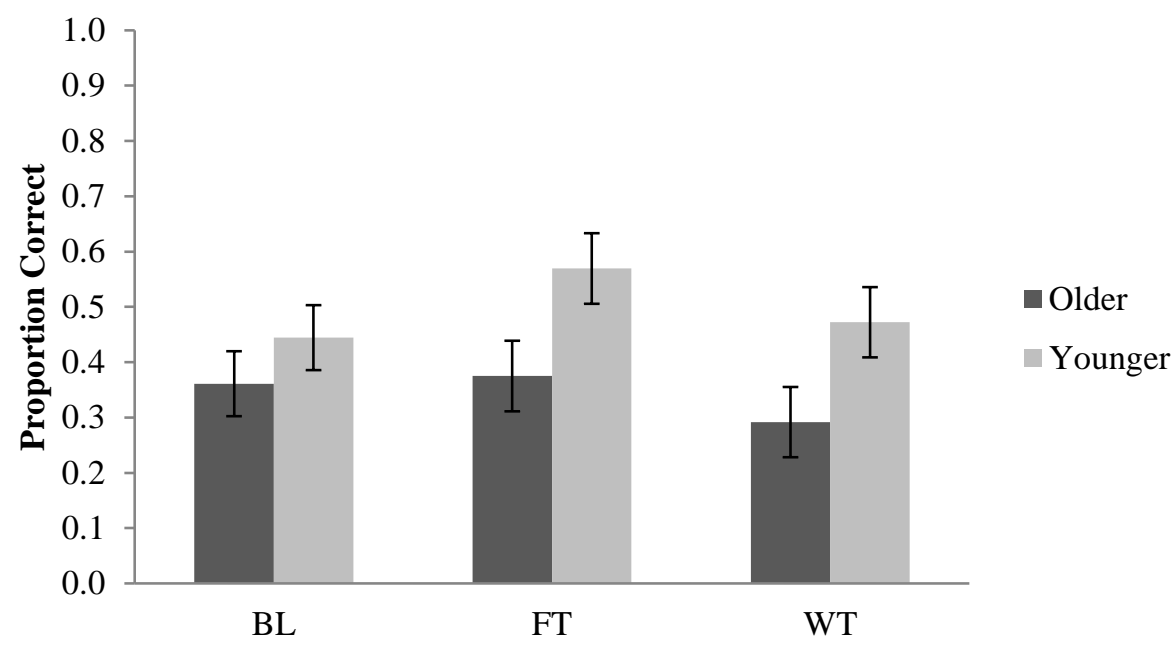

Figure 11. Irregular Time-Based Tasks

Mean proportion correct for each condition (BL = baseline; FT = future thinking; WT = whenthen, or implementation intentions). Standard errors are represented by the error bars attached to each column. 


\title{
Appendix A: Follow-Up Questionnaire - Experiment 1
}

\author{
Questionnaire: Goals
}

There are three sections to complete in this questionnaire.

1. Below you will find a list of words that describe who you might hope to be in the future. You can think of them as goal states, or as possible, desired versions of yourself. Please rank each word in order of importance for yourself. We recognize that this list may not capture all of the goals you have for yourself, and we also recognize that it might be difficult to rank them since some might be tied with others. Please just do your best to rank them anyway. Enter your numerical rating on the line to the right of each word. You must use all the numbers (1 through 8). There can be no ties and none left blank.

Scale:

$1=$ most important to $8=$ least important

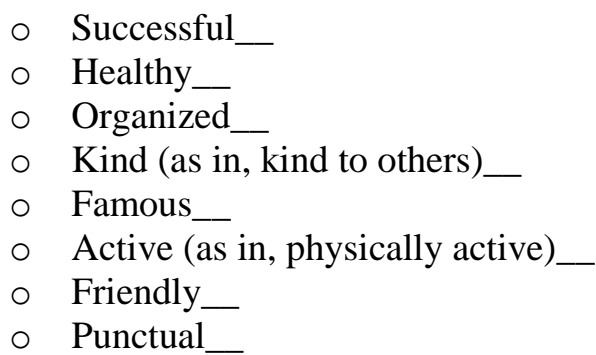

2. Below you will find additional goal themes that might be important to you. As best you can, please rank these themes in order of importance. Enter your numerical rating on the line to the right of each theme. Every theme must only have one rating. There can be no ties or themes left blank.

Scale:

$$
1=\text { most important to } 4=\text { least important }
$$

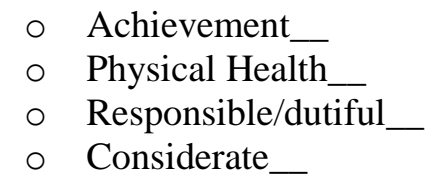

3. Please rate how well the word listed on the left is described by each of the four themes listed to its the right. Enter your numerical rating on the line to the left of each theme. Every theme must only have one rating. There can be no ties or entries left blank.

Scale:

$1=$ the word fits this theme the best, to $4=$ the word fits this theme the least

WORD

Successful:
THEME

_Achievement 
Physical Health

Responsible/dutiful

Considerate

Healthy:

Achievement

_Physical Health

_Responsible/dutiful

Considerate

Organized:

Achievement

_Physical Health

Responsible/dutiful

_ Considerate

Kind:

Achievement

_ Physical Health

_Responsible/dutiful

Considerate

Famous:

Achievement

_Physical Health

Responsible/dutiful

_ Considerate

Active:

_Achievement

_Physical Health

Responsible/dutiful

_ Considerate

Friendly:

Achievement

Physical Health

Responsible/dutiful

Considerate

Punctual:

Achievement

Physical Health

Responsible/dutiful

Considerate 


\section{Appendix B: Sample Narratives - Experiment 1}

\section{Participant: Older Adult \\ Cue word: Punctual}

OA: Okay. I am punctual. I am invariably on time, preferably 10 minutes early. I'm sorry I was born that way, I can't do anything about it. People find it very irritating because I am always there. I can just see myself, I can't help but arrive at a dinner party ahead of time, and I have to hold myself back. Everybody would be so grateful. So, I am going to imagine a get together on Friday afternoon as I always get together with a friend. What we call a "tea", it's not really tea. But - it's wine - but, we enjoy it very much. We've been getting together. It's the parent, the mother, my son's four-year-old's junior kindergarten class, so we get together, we've been getting together for almost forty years now. So, I am always there. I have to sit in the car, not to go to the door every time. So this date I am not going to be punctual, and I can just - and I do not think I am flattering myself to say that people would be very concerned. Concerned beyond belief if I did not get to that door at the appropriate time. I can see the looks of alarm on them sitting there. I can see astonishment. "Well, did [name] phone you?" "No, I haven't heard from her." "Well where is she? Does anybody know? Who should we call? Why isn't she there? She is always on time. Is she walking over or was she driving? What's going on? We better call somebody. Sure somebody knows where she is. Should we phone somebody?" End of story.

\section{Experimenter $^{22}:$ That's great. Any other details that you can imagine for this event?}

OA: I don't want to flatter myself, but I do believe there would be a high level of anxiety in that room, because I am always on time. It is very irritating. It's irritating to other people, it is. Who wants to be known for always being on time? Anyway, end of story.

\section{Participant: Younger adult}

Cue word: Successful

YA: Successful, okay. So this could happen on Friday. I'm like going to leave for this portaging trip. But I'm like sitting at my desk and I get an email about how I passed my exams and I like

\footnotetext{
${ }^{22}$ A prompt was provided because the participant stopped speaking before 2:20 minutes had elapsed.
} 
want to cry about it a little because I'm so happy. So I can see myself in like the living room and the like computer screen I can totally see what the email would look like from the professors, um... so congratulations and that I passed... and I just feel like this huge wave of relief like, I just feel like, "Oh my god thank god I don't have to do that horrible thing again". I'm like - so I have a like sense of euphoria and joy and my eyes water a little bit cause I'm just so happy because like I just remembered how much torture that process was, and um, so, yeah I'm just celebrating and I yell to my roommate and I tell her that I passed and then we open up this bad Champagne that we got for free that's in the fridge. It's like strawberry Champagne. So we're drinking that and remarking on how absolutely disgusting it is, um. But we're still really, really happy. Um, so we're just like kind of celebrating and just talking how we never ever have to do that again. Then I think about like writing about that or tweeting that or like Instagram-ing it cause then I'm like no that I'm not going to cause like that's kind of mean to other people. So I don't do that. But I'm still very happy inside. And then I just like, probably, do like, a hand stand to celebrate my happiness. That's the end of the story. 


\section{Appendix C: Consent \& Debriefing Forms - Experiment 1}

Younger Adults

\section{CONSENT AGREEMENT}

You are asked to participate in a research study. Before you give your consent to participate, please make sure you have read and understood the following information. We encourage you to ask questions if anything seems unclear. You will receive a copy of the consent form for your records.

Study Title: Future Thinking in Younger and Older Adults

Investigators: Julia Spaniol, $\mathrm{PhD}$, Department of Psychology, Ryerson University. Leann Lapp, MSc, MA, PhD candidate, Department of Psychology, Ryerson University.

Purpose of the Study: This is a study about future thinking in younger and older adults. We plan to recruit approximately 36 younger adults and 36 older adults.

The results of this study will contribute to Leann Lapp's $\mathrm{PhD}$ dissertation. This study is sponsored by NSERC.

\section{Description of the Study:}

- This study involves 1 session at Ryerson, which will last about 90 minutes (1.5 hours).

We will begin the session at Ryerson by asking you to complete several pencil and paper questionnaires about your demographics, your mood, and health-related information, as well as some questionnaires about vocabulary and symbol-drawing. Next, you will be guided to the computer, where you will imagine, out loud, several future scenarios in response to random cue words. You will provide several numerical ratings on the computer after generating each future scenario. Upon completion, you will complete another short pencil and paper questionnaire about your mood. The experimenter will explain the tasks to you in more detail before you begin and they will answer any questions you may have before signing this form.

What is Experimental in this Study: None of the procedures used in this study are experimental in nature. The only experimental aspect of this study is the gathering of information for the purpose of analysis.

Risks and Discomforts: Being involved in a study may be a new experience for you, and you may feel uncomfortable answering some of our questions. However, any potential discomfort is expected to be temporary and will not be greater than what you might experience in a typical day as a university student or at work. Remember that you do not have to answer all questions and that you may discontinue participation at any time with no penalty or loss of benefits to which you are entitled.

Benefits of the Study: There is no direct benefit to participants in this study other than learning about how a research study is conducted. However, the results of the study will provide important information about future thinking across the lifespan, which will benefit the cognitive aging research literature and potentially, society as a whole. 
Confidentiality: Each individual's results are confidential. Neither your identity nor any personal information will be available to anyone other than the investigators. Each participant will receive a numerical ID. Only the ID number will appear on electronic files data files as well as on hard-copy data forms and questionnaires. Only individuals involved in the research team will have access to a central password-protected electronic file that matches participant ID numbers with identifying information (name, date of birth, responses to demographic and health-related questions). This information is being kept in the event that a follow-up study is conducted in the future. No identifying information will be released or published without your informed consent.

All research records will be stored in a locked file cabinet in a locked laboratory. Electronic files will be password-protected and will be stored locally on a secure server on the Ryerson network. They will never be e-mailed or sent via file-sharing software. Electronic files will be kept for 5 years. Paper records will be destroyed after completion of the study.

\section{Incentives to Participate:}

- As you signed up for this study through SONA (i.e., the Intro Psychology Research Participant Pool), you will receive 1.5 credits towards your final mark in PSY102/202 for completion of the in-lab session. You will still receive the 1.5 credits even if you decide to discontinue your participation before the completion of the session.

Voluntary Nature of Participation: Your participation is voluntary. Your choice of whether or not to participate will not influence your future relations with Ryerson University. Please be aware that SONA participants have the option of a 'walk-through': if you decide to complete the study without allowing us to use your data, you will still receive course credit as compensation for your time.

This study has been reviewed and approved by the Research Ethics Board at Ryerson University. If you decide to participate, you are free to withdraw your consent and to stop your participation at any time without penalty or loss of benefits to which you are entitled. At any particular point in the study, you may refuse to answer any particular question or stop participation altogether.

If you have any questions about the research now, please ask. If you have questions about the research later, you may contact:

Dr. Julia Spaniol

jspaniol@psych.ryerson.ca

416-979-5000 (Ex: 2268)

Ms. Leann Lapp

leann.lapp@psych.ryerson.ca

If you have questions regarding your rights as a participant in this study, or you would like to speak to someone not connected to the research project, you may contact:

The Ryerson Research Ethics Board

c/o Office of the Vice President, Research and Innovation

Ryerson University

350 Victoria Street

Toronto, ON M5B 2K3

(416) 979-5042

If you any have questions about receiving your Psychology 102/202 credit for participation please contact: psychpool@ryerson.ca 


\section{Signature Section}

\section{Agreement:}

Your signature below indicates that you have read the information in this agreement and have had a chance to ask any questions you have about the study. Your signature also indicates that you agree to be in the study and have been told that you can change your mind and withdraw your consent to participate at any time. You have been given a copy of this agreement.

You have been told that by signing this consent agreement you are not giving up any of your legal rights.

Name of Participant (please print)

Signature of Participant

Date

$\square$ Please check this box if you would like to receive a copy of the final report of this research.

Signature of Investigator

Date 


\section{CONSENT AGREEMENT}

You are asked to participate in a research study. Before you give your consent to participate, please make sure you have read and understood the following information. We encourage you to ask questions if anything seems unclear. You will receive a copy of the consent form for your records.

Study Title: Future Thinking in Younger and Older Adults

Investigators: Julia Spaniol, PhD, Department of Psychology, Ryerson University. Leann Lapp, MSc, MA, PhD candidate, Department of Psychology, Ryerson University.

Purpose of the Study: This is a study about future thinking in younger and older adults. We plan to recruit approximately 36 younger adults and 36 older adults.

The results of this study will contribute to Leann Lapp's PhD dissertation. This study is sponsored by NSERC.

\section{Description of the Study:}

- This study involves 1 session at Ryerson, which will last about 120 minutes (2 hours).

We will begin the session at Ryerson by asking you to complete several pencil and paper questionnaires about your demographics, your mood, and health-related information, as well as some questionnaires about vocabulary and symbol-drawing. Next, you will be guided to the computer, where you will imagine, out loud, several future scenarios in response to random cue words. You will provide several numerical ratings on the computer after generating each future scenario. Upon completion, you will complete another short pencil and paper questionnaire about your mood. The experimenter will explain the tasks to you in more detail before you begin and they will answer any questions you may have before signing this form.

What is Experimental in this Study: None of the procedures used in this study are experimental in nature. The only experimental aspect of this study is the gathering of information for the purpose of analysis.

Risks and Discomforts: Being involved in a study may be a new experience for you, and you may feel uncomfortable answering some of our questions. However, any potential discomfort is expected to be temporary and will not be greater than what you might experience in a typical day as a university student or at work. Remember that you do not have to answer all questions and that you may discontinue participation at any time with no penalty or loss of benefits to which you are entitled.

Benefits of the Study: There is no direct benefit to participants in this study other than learning about how a research study is conducted. However, the results of the study will provide important information about future thinking across the lifespan, which will benefit the cognitive aging research literature and potentially, society as a whole.

Confidentiality: Each individual's results are confidential. Neither your identity nor any personal information will be available to anyone other than the investigators. Each participant will receive a numerical ID. Only the ID number will appear on electronic files data files as well as on hard-copy data 
forms and questionnaires. Only individuals involved in the research team will have access to a central password-protected electronic file that matches participant ID numbers with identifying information (name, date of birth, responses to demographic and health-related questions). This information is being kept in the event that a follow-up study is conducted in the future. No identifying information will be released or published without your informed consent.

All research records will be stored in a locked file cabinet in a locked laboratory. Electronic files will be password-protected and will be stored locally on a secure server on the Ryerson network. They will never be e-mailed or sent via file-sharing software. Electronic files will be kept for 5 years. Paper records will be destroyed after completion of the study.

\section{Incentives to Participate:}

You will receive compensation at a rate of $\$ 12$ per hour to cover your time and travel costs. This will be prorated if you take longer than the expected 2 hours.

Voluntary Nature of Participation: Your participation is voluntary. Your choice of whether or not to participate will not influence your future relations with Ryerson University.

This study has been reviewed and approved by the Research Ethics Board at Ryerson University. If you decide to participate, you are free to withdraw your consent and/or discontinue your participation at any time without penalty or loss of benefits to which you are entitled. At any particular point in the study, you may refuse to answer any particular question or stop participation altogether.

If you have any questions about the research now, please ask. If you have questions about the research later, you may contact:

Dr. Julia Spaniol

jspaniol@psych.ryerson.ca

416-979-5000 (Ex: 2268)

Ms. Leann Lapp

leann.lapp@psych.ryerson.ca

If you have questions regarding your rights as a participant in this study, or you would like to speak to someone not connected to the research project, you may contact:

The Ryerson Research Ethics Board

c/o Office of the Vice President, Research and Innovation

Ryerson University

350 Victoria Street

Toronto, ON M5B 2K3

(416) 979-5042 


\section{Signature Section}

\section{Agreement:}

Your signature below indicates that you have read the information in this agreement and have had a chance to ask any questions you have about the study. Your signature also indicates that you agree to be in the study and have been told that you can change your mind and withdraw your consent to participate at any time. You have been given a copy of this agreement.

You have been told that by signing this consent agreement you are not giving up any of your legal rights.

Name of Participant (please print)

Signature of Participant

Date

$\square$ Please check this box if you would like to receive a copy of the final report of this research.

Signature of Investigator

Date 


\section{Study Debriefing \\ Future Thinking in Younger and Older Adults}

This study is concerned with how younger and older adults compare on aspects of future thinking. Thinking about your future is an important human ability. For instance, we require future thinking to help us solve future problems and make plans. Research shows that future thinking relies on overlapping brain areas used to think about our pasts, suggesting that future thinking is significantly related to autobiographical memory. Newer lines of cognitive aging research are beginning to look at how motivational factors (such as personal goals) may influence certain human processes such as memory.

Main question:

Studies comparing autobiographical memory across the lifespan have indicated that younger and older adults show some differences when they remember their pasts as well as when they imagine their futures. Older adults tend to include more "semantic" information (facts, meanings, and knowledge) in their descriptions and fewer "episodic" details (indications of reexperiencing in a specific event in time and space) than younger adults. Nevertheless, newer lines of research have also showed that personal goals can influence aspects of certain mental processes.

Study summary:

In this study, you were given 8 , seemingly random cue words. However, the cues were in fact not random. They were selected from a previous study we did where we looked at which themes of personal goals are important to younger and older adults. For instance, many younger adults are concerned with goals related to achievement while many older adults hold goals related to the physical domain. Thus, we selected some cue words that were more relevant to older adults, some that were more significant for younger adults, and some that were important to both groups. In response to each cue, you were to generate a plausible, novel future scenario with as much detail as possible. After each response, you were asked to rate your scenario on various aspects such as vividness and emotional valence.

Study hypothesis:

We were interested to see if differences between age groups would depend on the type of cue word that was used. We expect that when older adults are prompted with themes that are more relevant to them, they will generate future scenarios in a way that is more detailed, vivid, and more similar to how younger adults describe future scenarios.

Why is this study important?

This study will tell us if basic thinking processes such as future, autobiographical thinking can be influenced by key motivational themes that change in importance across the lifespan. This will tell us more about how aging affects future thinking.

Thank you again for your participation! Please contact leann.lapp@ psych.ryerson.ca if you have further questions about this study.

Further reading: Addis, D., Wong, A., \& Schacter, D. (2012). Age-related changes in the episodic simulation of future events. Psychological Science, 19(1), 33-41. 


\section{Appendix D: Consent \& Debriefing Forms - Experiment 2}

Younger Adults

\section{CONSENT AGREEMENT}

You are asked to participate in a research study. Before you give your consent to participate, please make sure you have read and understood the following information. We encourage you to ask questions if anything seems unclear. You will receive a copy of the consent form for your records.

Study Title: Future Thinking in Younger and Older Adults

Investigators: Julia Spaniol, $\mathrm{PhD}$, Department of Psychology, Ryerson University. Leann Lapp, MSc, MA, PhD candidate, Department of Psychology, Ryerson University.

Purpose of the Study: This is a study about future thinking in younger and older adults. We plan to recruit approximately 36 younger adults and 36 older adults.

The results of this study will contribute to Leann Lapp's $\mathrm{PhD}$ dissertation. This study is sponsored by NSERC (Natural Sciences and Engineering Research Council of Canada), Canada's federal funding agency for university-based research.

\section{Description of the Study:}

- This study involves 1 session at Ryerson, which will last about 120 minutes (2 hours).

- We will begin the session at Ryerson by asking you to complete several pencil and paper questionnaires about your demographics, your mood, vocabulary, your imagination, and your memory. Next, you will be guided to the computer, where you will play a computerized board game that simulates activities you might complete in daily life. After some practice playing the game, the instructions will vary. For some of the board game activities, you will imagine yourself performing them before you actually do them while for other ones, you will just verbally state what the activities are that you must later do. Thus, parts of the board game will require you to use your imagination! The experimenter will explain the tasks to you in more detail before you begin and they will answer any questions you may have before signing this form.

What is Experimental in this Study: None of the procedures used in this study are experimental in nature. The only experimental aspect of this study is the gathering of information for the purpose of analysis.

Risks and Discomforts: Being involved in a study may be a new experience for you, and you may feel uncomfortable answering some of our questions. However, any potential discomfort is expected to be temporary and will not be greater than what you might experience in a typical day as a university student or at work. Remember that you do not have to answer all questions and that you may discontinue participation at any time with no penalty or loss of benefits to which you are entitled.

Benefits of the Study: There is no direct benefit to participants in this study other than learning about how a research study is conducted. However, the results of the study will provide important information about future thinking across the lifespan, which will benefit the cognitive aging research literature and potentially, society as a whole. 
Confidentiality: Each individual's results are confidential. Neither your identity nor any personal information will be available to anyone other than the investigators. Each participant will receive a numerical ID. Only the ID number will appear on electronic files data files as well as on hard-copy data forms and questionnaires. Only individuals involved in the research team will have access to a central password-protected electronic file that matches participant ID numbers with identifying information (name, date of birth, responses to demographic and health-related questions). This information is being kept in the event that a follow-up study is conducted in the future. No identifying information will be released or published without your informed consent.

All research records will be stored in a locked file cabinet in a locked laboratory. Electronic files will be password-protected and will be stored locally on a secure server on the Ryerson network. They will never be e-mailed or sent via file-sharing software. Electronic files will be kept for 5 years. Paper records will be destroyed after completion of the study.

\section{Incentives to Participate:}

- As you signed up for this study through SONA (i.e., the Intro Psychology Research Participant Pool), you will receive 2 credits towards your final mark in PSY102/202 for completion of the inlab session. You will still receive the 2 credits even if you decide to discontinue your participation before the completion of the session.

Voluntary Nature of Participation: Your participation is voluntary. Your choice of whether or not to participate will not influence your future relations with Ryerson University. Please be aware that SONA participants have the option of a 'walk-through': if you decide to complete the study without allowing us to use your data, you will still receive course credit as compensation for your time.

This study has been reviewed and approved by the Research Ethics Board at Ryerson University. If you decide to participate, you are free to withdraw your consent and to stop your participation at any time without penalty or loss of benefits to which you are entitled. At any particular point in the study, you may refuse to answer any particular question or stop participation altogether.

If you have any questions about the research now, please ask. If you have questions about the research later, you may contact:
Dr. Julia Spaniol
jspaniol@psych.ryerson.ca
416-979-5000 (Ex: 2268)
Ms. Leann Lapp
leann.lapp@psych.ryerson.ca

If you have questions regarding your rights as a participant in this study, or you would like to speak to someone not connected to the research project, you may contact:

The Ryerson Research Ethics Board

c/o Office of the Vice President, Research and Innovation

Ryerson University

350 Victoria Street

Toronto, ON M5B 2K3

(416) 979-5042

email: rebchair@ryerson.ca

If you any have questions about receiving your Psychology 102/202 credit for participation please contact: thepool@psych.ryerson.ca 


\section{$\underline{\text { Signature Section }}$}

\section{Agreement:}

Your signature below indicates that you have read the information in this agreement and have had a chance to ask any questions you have about the study. Your signature also indicates that you agree to be in the study and have been told that you can change your mind and withdraw your consent to participate at any time. You have been given a copy of this agreement.

You have been told that by signing this consent agreement you are not giving up any of your legal rights.

Name of Participant (please print) 


\section{CONSENT AGREEMENT}

You are asked to participate in a research study. Before you give your consent to participate, please make sure you have read and understood the following information. We encourage you to ask questions if anything seems unclear. You will receive a copy of the consent form for your records.

Study Title: Future Thinking in Younger and Older Adults

Investigators: Julia Spaniol, PhD, Department of Psychology, Ryerson University. Leann Lapp, MSc, MA, PhD candidate, Department of Psychology, Ryerson University.

Purpose of the Study: This is a study about future thinking in younger and older adults. We plan to recruit approximately 36 younger adults and 36 older adults.

The results of this study will contribute to Leann Lapp's PhD dissertation. This study is sponsored by NSERC (Natural Sciences and Engineering Research Council of Canada), Canada's federal funding agency for university-based research.

\section{Description of the Study:}

- This study involves 1 session at Ryerson, which will last about 150 minutes (2.5 hours).

- We will begin the session at Ryerson by asking you to complete several pencil and paper questionnaires about your demographics, your mood, vocabulary, your ability to generate words within a time limit, your imagination, and your memory. Next, you will be guided to the computer, where you will play a computerized board game that simulates activities you might complete in daily life. After some practice playing the game, the instructions will vary. For some of the board game activities, you will imagine yourself performing them before you actually do them while for other ones, you will just verbally state what the activities are that you must later do. Thus, parts of the board game will require you to use your imagination! The experimenter will explain the tasks to you in more detail before you begin and they will answer any questions you may have before signing this form.

What is Experimental in this Study: None of the procedures used in this study are experimental in nature. The only experimental aspect of this study is the gathering of information for the purpose of analysis.

Risks and Discomforts: Being involved in a study may be a new experience for you, and you may feel uncomfortable answering some of our questions. However, any potential discomfort is expected to be temporary and will not be greater than what you might experience in a typical day as a university student or at work. Remember that you do not have to answer all questions and that you may discontinue participation at any time with no penalty or loss of benefits to which you are entitled.

Benefits of the Study: There is no direct benefit to participants in this study other than learning about how a research study is conducted. However, the results of the study will provide important information about future thinking across the lifespan, which will benefit the cognitive aging research literature and potentially, society as a whole. 
Confidentiality: Each individual's results are confidential. Neither your identity nor any personal information will be available to anyone other than the investigators. Each participant will receive a numerical ID. Only the ID number will appear on electronic files data files as well as on hard-copy data forms and questionnaires. Only individuals involved in the research team will have access to a central password-protected electronic file that matches participant ID numbers with identifying information (name, date of birth, responses to demographic and health-related questions). This information is being kept in the event that a follow-up study is conducted in the future. No identifying information will be released or published without your informed consent.

All research records will be stored in a locked file cabinet in a locked laboratory. Electronic files will be password-protected and will be stored locally on a secure server on the Ryerson network. They will never be e-mailed or sent via file-sharing software. Electronic files will be kept for 5 years. Paper records will be destroyed after completion of the study.

\section{Incentives to Participate:}

You will receive $\$ 30$ compensation to cover your time and travel costs. This will be prorated if you take longer than the expected 2.5 hours.

Voluntary Nature of Participation: Your participation is voluntary. Your choice of whether or not to participate will not influence your future relations with Ryerson University.

This study has been reviewed and approved by the Research Ethics Board at Ryerson University. If you decide to participate, you are free to withdraw your consent and/or discontinue your participation at any time without penalty or loss of benefits to which you are entitled. At any particular point in the study, you may refuse to answer any particular question or stop participation altogether.

If you have any questions about the research now, please ask. If you have questions about the research later, you may contact:
Dr. Julia Spaniol
jspaniol@psych.ryerson.ca
416-979-5000 (Ex: 2268)
Ms. Leann Lapp
leann.lapp@psych.ryerson.ca

If you have questions regarding your rights as a participant in this study, or you would like to speak to someone not connected to the research project, you may contact:

The Ryerson Research Ethics Board

c/o Office of the Vice President, Research and Innovation

Ryerson University

350 Victoria Street

Toronto, ON M5B 2K3

(416) 979-5042

email: rebchair@ryerson.ca 


\section{$\underline{\text { Signature Section }}$}

\section{Agreement:}

Your signature below indicates that you have read the information in this agreement and have had a chance to ask any questions you have about the study. Your signature also indicates that you agree to be in the study and have been told that you can change your mind and withdraw your consent to participate at any time. You have been given a copy of this agreement.

You have been told that by signing this consent agreement you are not giving up any of your legal rights.

Name of Participant (please print)

Signature of Participant

Date

Signature of Investigator

Date 


\section{Study Debriefing}

Background: This study is concerned with how younger and older adults remember to complete daily activities. This type of memory is called prospective memory, or remembering to perform a planned action or intention at some future time point. Some common examples include deciding to buy bread later on your way home from work, or remembering to take your medication after breakfast every day. Sometimes, remembering to carry out these actions just "pops" into your mind when you see the cue (e.g. once you pass the grocery store, you remember you need to buy something), while at other times, you must consciously keep your attention on your planned intention or action. Difficulties with prospective memory are a common difficulty, especially among older adults. As such, there is great interest in understanding how prospective memory works and how it can be improved. Research suggests that forming simple but specific verbal statements (which are called "implementation intentions" (or the "when-then technique)) can help increase the success of your prospective memory. Early research also suggests that visualizing yourself carrying out the future action may also improve prospective memory, but very few studies have investigated this so far.

Main Study Question: In this study, you performed the Virtual Week, which is a computerized board game that involves prospective memory tasks on each virtual day. We wondered if imagining yourself doing the tasks before carrying them out would increase your likelihood of remembering to do them later. As a comparison, we also had you go through virtual days without any special instructions, while on other days, we asked you to form simple verbal statements ("when-then technique") about what you planned to do later that day.

Study hypotheses: We expect that on days where you imagined yourself carrying out the tasks, you would be more likely to remember to do them as compared to when you had no special instructions. We also expect that on days where you formed the verbal statements, your likelihood of carrying out the daily tasks would also be increased compared to baseline, but to a lesser degree than the imagination condition.

Why is this study important? This study will tell us if visualization can help improve prospective memory performance on a lab-based task. If this is the case, then future studies could investigate this strategy in real life activities outside the lab. It is also very possible that the strategy might not help with prospective memory, which would also be helpful for researchers trying to understand prospective memory. A null finding such as this would tell researchers that they should investigate different strategies to improve prospective memory.

Thank you again for your participation! Please contact leann.lapp@psych.ryerson.ca or jspaniol@psych.ryerson.ca (Dr. Julia Spaniol; supervisor) if you have further questions about this study. If you have questions regarding your rights as a participant in this study, or you would like to speak to someone not connected to the research project, you may contact:

The Ryerson Research Ethics Board c/o Office of the Vice President, Research and Innovation

Ryerson University

350 Victoria Street

Toronto, ON M5B 2K3 
Further reading: Altgassen, M., et al. (2015). Future thinking improves prospective memory performance and plan enactment in older adults. The Quarterly Journal of Experimental Psychology, 68(1), 192-204.

http://www.psychologicalscience.org/index.php/news/releases/when-we-forget-to-rememberfailures-in-prospective-memory-range-from-annoying-to-lethal.html 


\title{
Appendix E: Strategy Instruction Slides - Experiment 2
}

\author{
Future Thinking E-Prime Tutorial Slides
}

\begin{abstract}
For the next two days of the Virtual Week, you will be provided with additional instructions for the new tasks that you are assigned each day.

These instructions will not apply to the tasks you repeat everyday (e.g. taking your medication or checking your lung function); rather, they will apply only to the NEW TASKS that become assigned to you as the day proceeds.
\end{abstract}

\begin{abstract}
As a reminder, you'll see a prompt under the NEW TASKS
\end{abstract} which will tell you when to use the new instructions.

Press the spacebar to continue instructions.

When you are assigned a new task for the day, use the "Future Thinking Technique."

This involves taking $\mathbf{3 0}$ seconds to close your eyes and imagine yourself carrying out this activity. Imagine yourself completing this activity as if you were doing it in your everyday life (rather than in the game or at the lab).

Press the spacebar for an example.

How would this scenario unfold? You might imagine yourself passing the post office, walking in, and going through the procedure of buying envelopes.

Describe this imagined event aloud.

We want you to imagine the specific steps of this event.

To help enrich your imagined scenario, try using your five senses: What might you see around you? What might you hear? What might you think or feel? What might you smell, taste or touch?

Press the spacebar to continue instructions.

You will be asked to describe what you are imagining out loud. The experimenter will tell you when your time is up. If there is extra time remaining, the experimenter will also ask if you have any additional details.

You will practice here before returning to the Virtual Week so that you get the hang of using the "Future Thinking Technique."

Do you have any questions?

Press the spacebar to begin practice trials.
Your first appointment for Wednesday.

-take dog for walk when you get home

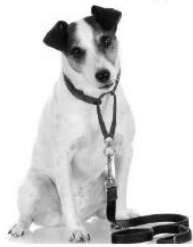

FUTURE THINKING TECHNIQUE

Press the spacebar to continue.
Your second appointment for Wednesday.

-bring car to mechanic's garage at 11:30am

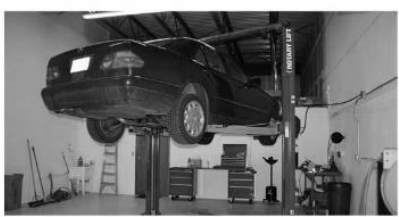

FUTURE THINKING TECHNIQUE

Press the spacebar to continue. 


\section{When-Then (Implementation Intentions) E-Prime Tutorial Slides}

For the next two days of the Virtual Week, you will be provided with additional instructions for the new tasks that you are assigned each day.

These instructions will not apply to the tasks you repeat everyday (e.g. taking your medication or checking your lung function); rather, they will apply only to the NEW TASKS that become assigned to you as the day proceeds.

As a reminder, you'll see a prompt under the NEW TASKS which will tell you when to use the new instructions.

Press the spacebar to continue instructions.
When you are assigned a new task for the day, use the "WhenThen Technique."

This involves forming a verbal statement taking the following format:

"When situation $\mathrm{X}$ arises, then I will perform specific task $\mathrm{Y}$."

Press the spacebar for an example.

You will practice here before returning to the Virtual Week so that you get the hang of coming up with these "WhenThen" statements.

If you were assigned to call your dentist's office at 10am to make an appointment, you would say:

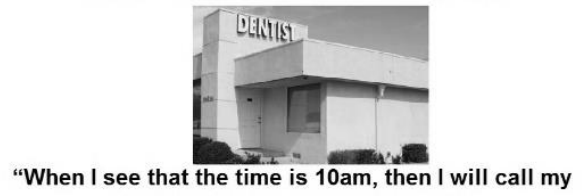
dentist to make an appointment."

Press the spacebar to continue.

Press the spacebar to begin practice trials.

Your first appointment for Friday.

Your second appointment for Friday.

-check mailbox for package at 5:00pm

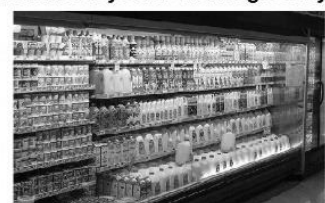

WHEN THEN TECHNIQUE

Press the spacebar to continue.

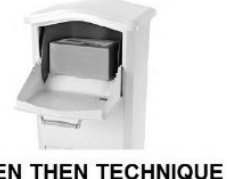

Press the spacebar to continue. 


\section{References}

Abram, M., Picard, L., Navarro, B., \& Piolino, P. (2014). Mechanisms of remembering the past and imagining the future - New data from autobiographical memory tasks in a lifespan approach. Consciousness and Cognition, 29, 76-89. doi:10.1016/j.concog.2014.07.011

Adams, C., Smith, M. C., Nyquist, L., \& Perlmutter, M. (1997). Adult age-group differences in recall for the literal and interpretive meanings of narrative text. The Journals of Gerontology: Series B: Psychological Sciences and Social Sciences,52B, P187-P195.

Adams, C., Smith, M. C., Pasupathi, M., \& Vitolo, L. (2002). Social context effects on story recall in older and younger women: Does the listener make a difference? The Journals of Gerontology: Series B: Psychological Sciences and Social Sciences,57B, P28-P40. doi:10.1093/geronb/57.1.P28

Addis, D. R., Musicaro, R., Pan, L., \& Schacter, D. L. (2010). Episodic simulation of past and future events in older adults: Evidence from an experimental recombination task. Psychology and Aging, 25, 369-376. doi:10.1037/a0017280

Addis, D. R., Roberts, R. P., \& Schacter, D. L. (2011). Age-related neural changes in autobiographical remembering and imagining. Neuropsychologia, 49, 3656-3669. doi:10.1016/j.neuropsychologia.2011.09.021

Addis, D. R., Sacchetti, D. C., Ally, B. A., Budson, A. E., \& Schacter, D. L. (2009). Episodic simulation of future events is impaired in mild Alzheimer's disease. Neuropsychologia, 47, 2660-2671. doi:10.1016/j.neuropsychologia.2009.05.018

Addis, D. R., Wong, A. T., \& Schacter, D. L. (2007). Remembering the past and imagining the future: Common and distinct neural substrates during event construction and elaboration. Neuropsychologia, 45, 1363-1377. doi:10.1016/j.neuropsychologia.2006.10.016 
Addis, D. R., Wong, A. T., \& Schacter, D. L. (2008). Age-related changes in the episodic simulation of future events. Psychological Science, 19, 33-41. doi:10.1111/j.14679280.2008.02043.x

Alea, N., \& Bluck, S. (2003). Why are you telling me that? A conceptual model of the social function of autobiographical memory. Memory, 11, 165-178. doi:10.1080/741938207

Altgassen, M., Kretschmer, A., \& Schnitzspahn, K. M. (2016). Future thinking instructions improve prospective memory performance in adolescents. Child Neuropsychology, 1-18. doi:10.1080/09297049.2016.1158247

Altgassen, M., Rendell, P. G., Bernhard, A., Henry, J. D., Bailey, P. E., Phillips, L. H., \& Kliegel, M. (2015). Future thinking improves prospective memory performance and plan enactment in older adults. The Quarterly Journal of Experimental Psychology, 68, 192204. doi:10.1080/17470218.2014.956127

Baltes, P. B. (1997). On the incomplete architecture of human ontogeny: Selection, optimization, and compensation as foundation of developmental theory. American Psychologist, 52, 366-380. doi:10.1037/0003-066X.52.4.366

Bandura, A. (1986). Social foundations of thought and action: A social cognitive theory. Englewood Cliffs, NJ: Prentice-Hall, Inc.

Barsics, C., Van, d. L., \& D'Argembeau, A. (2016). Frequency, characteristics, and perceived functions of emotional future thinking in daily life. The Quarterly Journal of Experimental Psychology, 69, 217-233. doi:10.1080/17470218.2015.1051560

Bartlett, F.C. (1932). Remembering. Cambridge, England: Cambridge University Press.

Beck, J. S. (1976). Cognitive therapy and the emotional disorders. New York: International Universities Press. 
Bélanger-Gravel, A., Godin, G., Bilodeau, A., \& Poirier, P. (2013). The effect of implementation intentions on physical activity among obese older adults: A randomised control study. Psychology \& Health, 28, 217-233. doi:10.1080/08870446.2012.723711

Benoit, R. G., \& Schacter, D. L. (2015). Specifying the core network supporting episodic simulation and episodic memory by activation likelihood estimation. Neuropsychologia, 75, 450-457. doi:10.1016/j.neuropsychologia.2015.06.034

Berntsen, D., \& Bohn, A. (2010). Remembering and forecasting: The relation between autobiographical memory and episodic future thinking. Memory \& Cognition, 38, 265278. doi:10.3758/MC.38.3.265

Berntsen, D., \& Jacobsen, A. S. (2008). Involuntary (spontaneous) mental time travel into the past and future. Consciousness and Cognition: An International Journal, 17, 1093-1104. doi:10.1016/j.concog.2008.03.001

Berntsen, D., \& Rubin, D. C. (2004). Cultural life scripts structure recall from autobiographical memory. Memory \& Cognition,32, 427-442. doi:10.3758/BF03195836

Bluck, S., \& Alea, N. (2011). Crafting the TALE: Construction of a measure to assess the functions of autobiographical remembering. Memory, 19, 470-486. doi:10.1080/09658211.2011.590500

Bowie, C. R., \& Harvey, P. D. (2006). Administration and interpretation of the Trail Making Test. Nature Protocols, 1, 2277-2281.

Brandimonte, M., Einstein, G. O., \& McDaniel, M. A. (Eds.). (1996). Prospective memory: Theory and applications. Mahwah, NJ: Erlbaum.

Braver, T.S., Barch, D.M., Keys, B.A., Carter, C.S., Cohen, J.D., Kaye, J.A., ... \& Jagust, W.J. (2001). Context processing in older adults: Evidence for a theory relating cognitive 
control to neurobiology in healthy aging. Journal of Experimental Psychology: General, 130, 746-763.

Brewer, G. A., Knight, J., Thadeus Meeks, J., \& Marsh, R. L. (2011). On the role of imagery in event-based prospective memory. Consciousness and Cognition: An International Journal, 20, 901-907.

Buckner, R. L., \& Carroll, D. C. (2007). Self-projection and the brain. Trends in Cognitive Sciences, 11, 49-57. doi:10.1016/j.tics.2006.11.004

Burgess, P. W., Gonen-Yaacovi, G., \& Volle, E. (2011). Functional neuroimaging studies of prospective memory: What have we learnt so far? Neuropsychologia, 49, 2246-2257. doi:10.1016/j.neuropsychologia.2011.02.014

Burkard, C., Rochat, L., Blum, A., Emmenegger, J., Van der Linden, A.C., \& Van der Linden, M. (2014). A daily-life-oriented intervention to improve prospective memory and goaldirected behaviour in ageing: A pilot study. Neuropsychological Rehabilitation, 24, 266295. doi:10.1080/09602011.2014.887023

Burkard, C., Rochat, L., Van, d. L., Gold, G., \& Van, d. L. (2014). Is working memory necessary for implementation intentions to enhance prospective memory in older adults with cognitive problems? Journal of Applied Research in Memory and Cognition, 3, 37-43. doi:10.1016/j.jarmac.2014.01.004

Butler, M., Retzlaff, P., \& Vanderploeg, R. (1991). Neuropsychological test usage. Professional Psychology Research and Practice, 22, 510-512. doi:10.1037/0735-7028.22.6.510

Cabeza, R., Anderson, N. D., Houle, S., Mangels, J. A., \& Nyberg, L. (2000). Age-related differences in neural activity during item and temporal-order memory retrieval: A 
positron emission tomography study. Journal of Cognitive Neuroscience, 12, 197-206. doi:10.1162/089892900561832

Carstensen, L. L., Isaacowitz, D. M., \& Charles, S. T. (1999). Taking time seriously: A theory of socioemotional selectivity. American Psychologist, 54, 165-181. doi:10.1037/0003066X.54.3.165

Carstensen, L. L., \& Mikels, J. A. (2005). At the intersection of emotion and cognition: Aging and the positivity effect. Current Directions in Psychological Science, 14, 117-121. doi:10.1111/j.0963-7214.2005.00348.x

Carstensen, L. L., Mikels, J. A., \& Mather, M. (2006). Aging and the intersection of cognition, motivation, and emotion. In J. E. Birren, \& K. W. Schaire (Eds.), 6th ed.; Handbook of the psychology of aging ( pp. 343-362, Chapter xxi) Amsterdam: Elsevier. doi:10.1016/B978-012101264-9/50018-5

Charles, S. T., Mather, M., \& Carstensen, L. L. (2003). Aging and emotional memory: The forgettable nature of negative images for older adults. Journal of Experimental Psychology: General, 132, 310-324. doi:10.1037/0096-3445.132.2.310

Chasteen, A. L., Park, D. C., \& Schwarz, N. (2001). Implementation intentions and facilitation of prospective memory. Psychological Science, 12, 457-461.

Chen, X., Wang, Y., Liu, L., Cui, J., Gan, M., Shum, D. H. K., \& Chan, R. C. K. (2015). The effect of implementation intention on prospective memory: A systematic and metaanalytic review. Psychiatry Research, 226, 14-22. doi:10.1016/j.psychres.2015.01.011

Chessell, Z. J., Rathbone, C. J., Souchay, C., Charlesworth, L., \& Moulin, C. J. A. (2014). Autobiographical memory, past and future events, and self-images in younger and older adults. Self and Identity, 13, 380-397. doi:10.1080/15298868.2013.836132 
Christian, B. M., Miles, L. K., Fung, F. H. K., Best, S., \& Macrae, C. N. (2013). The shape of things to come: Exploring goal-directed prospection. Consciousness and Cognition, 22, 471-478. doi:10.1016/j.concog.2013.02.002

Cohen, A., \& Gollwitzer, P. M. (2008). The cost of remembering to remember: Cognitive load and implementation intentions influence ongoing task performance. Prospective memory: Cognitive, neuroscience, developmental, and applied perspectives. (pp. 367-390) New York: Erlbaum.

Cole, S. (2016). A timely dose of theory in future thinking research. The Quarterly Journal of Experimental Psychology. 70, 347-349. doi: 10.1080/17470218.2016.1245347

Cole, S. N., Morrison, C. M., \& Conway, M. A. (2013). Episodic future thinking: Linking neuropsychological performance with episodic detail in young and old adults. The Quarterly Journal of Experimental Psychology, 66, 1687-1706.

doi:10.1080/17470218.2012.758157

Cole, S. N., Staugaard, S., \& Berntsen, D. (2016). Inducing involuntary and voluntary mental time travel using a laboratory paradigm. Memory \& Cognition, 44, 376-389. doi:10.3758/s13421-015-0564-9

Conway, M. A. (2005). Memory and the self. Journal of Memory and Language, 53, 594-628. doi:10.1016/j.jml.2005.08.005

Conway, M. A., \& Holmes, A. (2004). Psychosocial stages and the accessibility of autobiographical memories across the life cycle. Journal of Personality, 72, 461-480. doi:10.1111/j.0022-3506.2004.00269.x

Conway, M.A., \& Loveday, C. (2015). Remembering, imagining, false memories and personal meanings. Consciousness and Cognition, 33, 574-581. doi:10/1016/j.concog.2014-12.002 
Conway, M. A., Loveday, C., \& Cole, S. N. (2016). The remembering-imagining system. Memory Studies, 9, 256-265. doi:10.1177/1750698016645231

Conway, M. A., \& Pleydell-Pearce, C. (2000). The construction of autobiographical memories in the self-memory system. Psychological Review, 107, 261-288. doi:10.1037/0033295X.107.2.261

Correia, S.P.C., Dickinson, A., \& Clayton, N. (2007). Western scrub-jays anticipate future needs independently of their current motivational states. Current Biology, 17, 856-861. doi:10.1016/j.cub.2007.03.063

Craik, E I. M. (1983). On the transfer of information from temporary to permanent memory. Philosophical Transactions of the Royal Society of London, B302, 341-359

Craik, F.I.M. (1986). A functional account of age differences in memory. In F. Klix \& H. Hagendorf (Eds.), Human memory and cognitive capabilities, mechanisms, and performances (pp. 409-422). North Holland: Elsevier Science Publishers B.V.

Craik, F. I. M., \& Grady, C. L. (2002). Aging, memory, and frontal lobe functioning. New York: Oxford University Press. doi:10.1093/acprof:oso/9780195134971.003.0031

Craik, F.I.M. \& Salthouse, T.A. (Eds.). (2000). The handbook of aging and cognition. Mahwah, N.J.: Erlbaum.

Cross, S., \& Markus, H. (1991). Possible selves across the life span. Human Development, 34, $230-255$.

D'Argembeau, A., Lardi, C., \& Van de Lindsen (2012). Self-defining future projections: Exploring the identity function of thinking about the future. Memory, 20, 110-120. doi:10.1080/09658211.2011.647697 
D'Argembeau, A., \& Mathy, A. (2011). Tracking the construction of episodic future thoughts. Journal of Experimental Psychology: General, 140, 258-271. doi:10.1037/a0022581

D'Argembeau, A., Ortoleva, C., Jumentier, S., \& Van der Linden, M. (2010). Component processes underlying future thinking. Memory \& Cognition, 38, 809-819. doi:10.3758/MC.38.6.809

D'Argembeau, A., Renaud, O., \& Van der Linden, M. (2011). Frequency, characteristics and functions of future-oriented thoughts in daily life. Applied Cognitive Psychology, 25, 96103. doi:10.1002/acp.1647

D'Argembeau, A., \& Van der Linden. (2006). Individual differences in the phenomenology of mental time travel: The effect of vivid visual imagery and emotion regulation strategies. Consciousness and Cognition: An International Journal, 15, 342-350. doi:10.1016/j.concog.2005.09.001

Demblon, J., \& D'Argembeau, A. (2014). The organization of prospective thinking: Evidence of event clusters in freely generated future thoughts. Consciousness and Cognition: An International Journal, 24, 75-83. doi:10.1016/j.concog.2014.01.002

Duval, C., Desgranges, B., Sayette, d. L., Belliard, S., Eustache, F., \& Piolino, P. (2012). What happens to personal identity when semantic knowledge degrades? A study of the self and autobiographical memory in semantic dementia. Neuropsychologia, 50, 254-265. doi:10.1016/j.neuropsychologia.2011.11.019

Ebner, N. C., Freund, A. M., \& Baltes, P. B. (2006). Developmental changes in personal goal orientation from young to late adulthood: From striving for gains to maintenance and prevention of losses. Psychology and Aging, 21, 664-678. doi:10.1037/08827974.21.4.664 
Ebner, N. C., Riediger, M., \& Lindenberger, U. (2009). Schema reliance for developmental goals increases from early to late adulthood: Improvement for the young, loss prevention for the old. Psychology and Aging, 24, 310-323. doi:10.1037/a0015430

Einstein, G. O., \& McDaniel, M. A. (1990). Normal aging and prospective memory. Journal of Experimental Psychology: Learning, Memory, and Cognition, 16, 717-726. doi:10.1037/0278-7393.16.4.717

Einstein, G. O., \& McDaniel, M. A. (1996). Retrieval processes in prospective memory: Theoretical approaches and some new empirical findings. Prospective memory: Theory and applications. (pp. 115-141) Mahwah, NJ: Erlbaum.

El Haj, M., Antoine, P., \& Kapogiannis, D. (2015). Similarity between remembering the past and imagining the future in Alzheimer's disease: Implication of episodic memory. Neuropsychologia, 66, 119-125. doi:10.1016/j.neuropsychologia.2014.11.015

Faude-Koivisto, T., Wuerz, D., \& Gollwitzer, P. M. (2009). Implementation intentions: The mental representations and cognitive procedures of if-then planning. Handbook of imagination and mental simulation. (pp. 69-86) Psychology Press, New York, NY.

Faytell, M. P., Doyle, K. L., Naar-King, S., Outlaw, A. Y., Nichols, S. L., Casaletto, K. B., \& Woods, S. P. (2015). Visualisation of future task performance improves naturalistic prospective memory for some younger adults living with HIV disease. Neuropsychological Rehabilitation. doi:10.1080/09602011.2015.1122636

Fish, J., Wilson, B. A., \& Manly, T. (2010). The assessment and rehabilitation of prospective memory problems in people with neurological disorders: A review. Neuropsychological Rehabilitation, 20, 161-179. doi:10.1080/09602010903126029 
Folstein, M. F., Folstein, S. E., \& McHugh, P. R. (1975). Mini-mental state: A practical method for grading the cognitive state of patients for the clinician. Journal of Psychiatric Research, 12, 189-198. doi:10.1016/0022-3956(75)90026-6

Folstein, M. F., Folstein, S. E., McHugh, P. R., \& Fanjiang, G. (2001). Mini-Mental State Examination: User's Guide. Odessa, FL: Psychological Assessment Resources.

Frazier, L. D., Cotrell, V., \& Hooker, K. (2003). Possible selves and illness: A comparison of individuals with Parkinson's disease, early-stage Alzheimer's disease, and healthy older adults. International Journal of Behavioral Development, 27, 1-11. doi:10.1080/01650250143000526

Freund, A. M., \& Ebner, N. C. (2005). The aging self: Shifting from promoting gains to balancing losses. In W. Greve, K. Rothermund, \& D. Wentura (Eds.), The adaptive self: Personal continuity and intentional self-development. (pp. 185-202, Chapter 10). Ashland, OH: Hogrefe \& Huber.

Gaesser, B., Sacchetti, D. C., Addis, D. R., \& Schacter, D. L. (2011). Characterizing age-related changes in remembering the past and imagining the future. Psychology and Aging, 26, 80-84. doi:10.1037/a0021054

Gamboz, N., De Vito, S., Brandimonte, M. A., Pappalardo, S., Galeone, F., Iavarone, A., \& Della Sala, S. (2010). Episodic future thinking in amnesic mild cognitive impairment. Neuropsychologia, 48, 2091-2097. doi:10.1016/j.neuropsychologia.2010.03.030

Gerlach, K. D., Spreng, R. N., Gilmore, A. W., \& Schacter, D. L. (2011). Solving future problems: Default network and executive activity associated with goal-directed mental simulations. NeuroImage, 55, 1816-1824. doi:10.1016/j.neuroimage.2011.01.030 
Giovanello, K. S., Schnyer, D. M., \& Verfaellie, M. (2004). A critical role for the anterior hippocampus in relational memory: Evidence from an fMRI study comparing associative and item recognition. Hippocampus, 14, 5-8. doi:10.1002/hipo.10182

Giovagnoli, A. R., Del Pesce, M., Mascheroni, S., Simoncelli, M., Laiacona, M., \& Capitani, E. (1996). Trail Making Test: Normative values from 287 normal adult controls. The Italian Journal of Neurological Sciences, 17, 305-309. doi:10.1007/BF01997792

Gloster, A. T., Rhoades, H. M., Novy, D., Klotsche, J., Senior, A., Kunik, M., . . Stanley, M. A. (2008). Psychometric properties of the Depression Anxiety and Stress Scale-21 in older primary care patients. Journal of Affective Disorders, 110, 248-259. doi:10.1016/j.jad.2008.01.023

Gollwitzer, P. M. (1990). Action phases and mind-sets. Handbook of motivation and cognition: Foundations of social behavior (Vol. 2, pp. 53-92) New York: Guilford Press.

Gollwitzer, P. M. (1999). Implementation intentions: Strong effects of simple plans. American Psychologist, 54, 493-503. doi:10.1037/0003-066X.54.7.493

Gollwitzer, P. M., \& Sheeran, P. (2006). Implementation intentions and goal achievement: A meta-analysis of effects and processes. Advances in experimental social psychology (Vol 38, pp. 69-119) San Diego, CA: Elsevier. doi:10.1016/S0065-2601(06)38002-1

Gonen-Yaacovi, G., \& Burgess, P. W. (2012). Prospective memory: The future for future intentions. Psychologica Belgica, 52, 173-204.

Grady, C. (2012). Trends in neurocognitive aging. Nature Review Neuroscience, 13, 491-505. doi:10.1038/nrn3256.

Graf, P., \& Uttl, B. (2001). Prospective memory: A new focus for research. Consciousness and Cognition: An International Journal, 10, 437-450. doi:10.1006/ccog.2001.0504 
Greitemeyer, T., \& Würz, D. (2005). Mental simulation and the achievement of health goals: The role of goal difficulty.Imagination, Cognition and Personality, 25, 239-251. doi:10.2190/D4UA-RQFQ-0H5T-W9YY

Greenwood, P. M., \& Parasuraman, R. (2010). Neuronal and cognitive plasticity: A neurocognitive framework for ameliorating cognitive aging. Frontiers in Aging Neuroscience, 2, 14. doi:10.3389/fnagi.2010.00150

Griffiths, A., Hill, R., Morgan, C., Rendell, P. G., Karimi, K., Wanagaratne, S., \& Curran, H. V. (2012). Prospective memory and future event simulation in individuals with alcohol dependence. Addiction, 107, 1809-1816. doi:10.1111/j.1360-0443.2012.03941.x

Grilli, M. D., \& Glisky, E. L. (2010). Self-imagining enhances recognition memory in memoryimpaired individuals with neurological damage. Neuropsychology, 24, 698-710. doi:10.1037/a0020318

Grilli, M. D., \& Glisky, E. L. (2011). The self-imagination effect: Benefits of a self-referential encoding strategy on cued recall in memory-impaired individuals with neurological damage. Journal of the International Neuropsychological Society, 17, 929-933.

Grilli, M. D., \& McFarland, C. P. (2011). Imagine that: Self-imagination improves prospective memory in memory-impaired individuals with neurological damage. Neuropsychological Rehabilitation, 21, 847-859.

Gross, A. L., Parisi, J. M., Spira, A. P., Kueider, A. M., Ko, J. Y., Saczynski, J. S., . . Rebok, G. W. (2012). Memory training interventions for older adults: A meta-analysis. Aging \& Mental Health, 16, 722-734. doi:10.1080/13607863.2012.667783 
Grühn, D., Sharifian, N., \& Chu, Q. (2016). The limits of a limited future time perspective in explaining age differences in emotional functioning. Psychology and Aging, 31, 583-593. doi:10.1037/pag0000060

Grysman, A., Prabhakar, J., Anglin, S.M., \& Hudson, J.A. (2010). Self-enhancement and the life script in future thinking across the lifespan. Memory. doi:10.1080/09658211.2014.927505.

Gutchess, A. H., Kensinger, E. A., Yoon, C., \& Schacter, D. L. (2007). Ageing and the selfreference effect in memory. Memory, 15, 822-837. doi:10.1080/09658210701701394

Hamami, A., Serbun, S. J., \& Gutchess, A. H. (2011). Self-referencing enhances memory specificity with age. Psychology and Aging, 26, 636-646. doi:10.1037/a0022626

Hartman, D. E. (2009). Test review Wechsler Adult Intelligence Scale IV (WAIS IV): Return of the gold standard. Applied Neuropsychology, 16, 85-87. http://dx.doi.org/10.1080/09084280802644466

Henry, J. D., MacLeod, M. S., Phillips, L. H., \& Crawford, J. R. (2004). A meta-analytic review of prospective memory and aging. Psychology and Aging, 19, 27-39. doi:10.1037/08827974.19.1.27

Henry, J. D., Rendell, P. G., Kliegel, M., \& Altgassen, M. (2007). Prospective memory in schizophrenia: Primary or secondary impairment? Schizophrenia Research, 95, 179-185. doi:10.1016/j.schres.2007.06.003

Hering, A., Phillips, L.H., \& Kliegel, M. (2014). Importance effects on age differences in performance in event-based prospective memory. Gerontology, 60, 73-78. doi: $10.1159 / 000355057$ 
Hering, A., Rendell, P. G., Rose, N. S., Schnitzspahn, K. M., \& Kliegel, M. (2014). Prospective memory training in older adults and its relevance for successful aging. Psychological Research, 78, 892-904. doi:10.1007/s00426-014-0566-4

Hess, T. M. (2005). Memory and aging in context. Psychological Bulletin, 131, 383-406. doi:10.1037/0033-2909.131.3.383

Hess, T.M. (2006). Attitudes towards aging and their effects on behavior. In J.E. Birren \& K.W. Schaie (Eds.), Handbook of the psychology of aging (6 ${ }^{\text {th }}$ ed., pp. 379-406). San Diego: Academic Press.

Hess, T. M. (2014). Selective engagement of cognitive resources: Motivational influences on older adults' cognitive functioning. Perspectives on Psychological Science, 9, 388-407. doi:10.1177/1745691614527465

Higgins, E. T. (1997). Beyond pleasure and pain. American Psychologist, 52, 1280-1300. doi:10.1037/0003-066X.52.12.1280

Holmes, A., \& Conway, M. A. (1999). Generation identity and the reminiscence bump: Memory for public and private events. Journal of Adult Development, 6, 21-34. doi:10.1023/A:1021620224085

Hooker, K. (1992). Possible selves and perceived health in older adults and college students. Journal of Gerontology,47(2), P85-P95.

Ihle, A., Hering, A., Mahy, C. E. V., Bisiacchi, P. S., \& Kliegel, M. (2013). Adult age differences, response management, and cue focality in event-based prospective memory: A meta-analysis on the role of task order specificity. Psychology and Aging,28, 714-720. doi:10.1037/a0033653 
Irish, M., Addis, D. R., Hodges, J. R., \& Piguet, O. (2012). Exploring the content and quality of episodic future simulations in semantic dementia. Neuropsychologia, 50, 3488-3495. doi:10.1016/j.neuropsychologia.2012.09.012

Irish, M., \& Piolino, P. (2016). Impaired capacity for prospection in the dementias-Theoretical and clinical implications. British Journal of Clinical Psychology, 55, 49-68. doi:10.1111/bjc. 12090

Jason, L. A., Schade, J., Furo, L., Reichler, A., \& al, e. (1989). Time orientation: Past, present, and future perceptions. Psychological Reports, 64, 1199-1205. doi:10.2466/pr0.1989.64.3c.1199

Jing, H. G., Madore, K. P., \& Schacter, D. L. (2016). Worrying about the future: An episodic specificity induction impacts problem solving, reappraisal, and well-being. Journal of Experimental Psychology: General, 145, 402-418. doi:10.1037/xge0000142

Kelemen, W. L., Weinberg, W. B., Alford, H. S., Mulvey, E. K., \& Kaeochinda, K. F. (2006). Improving the reliability of event-based laboratory tests of prospective memory. Psychonomic Bulletin \& Review, 13, 1028-1032. doi:10.3758/BF03213920

Kennedy, Q., Mather, M., \& Carstensen, L. L. (2004). The role of motivation in the age-related positivity effect in autobiographical memory. Psychological Science, 15, 208-214. doi:10.1111/j.0956-7976.2004.01503011.x

Klein, S. B. (2012). Self, memory, and the self-reference effect: An examination of conceptual and methodological issues. Personality and Social Psychology Review, 16, 283-300. doi: $10.1177 / 1088868311434214$ 
Kliegel, M., Altgassen, M., Hering, A., \& Rose, N. S. (2011). A process-model based approach to prospective memory impairment in Parkinson's disease. Neuropsychologia, 49, 21662177. doi:10.1016/j.neuropsychologia.2011.01.024

Kliegel, M., \& Jäger, T. (2006). Delayed-execute prospective memory performance: The effects of age and working memory. Developmental Neuropsychology, 30, 819-843. doi:10.1207/s15326942dn3003_4

Kliegel, M., Jäger, T., \& Phillips, L. H. (2008). Adult age differences in event-based prospective memory: A meta-analysis on the role of focal versus nonfocal cues. Psychology and Aging, 23, 203-208. doi:10.1037/0882-7974.23.1.203

Kretschmer-Trendowicz, A., Ellis, J. A., \& Altgassen, M. (2016). Effects of episodic future thinking and self-projection on children's prospective memory performance. PloS one, 11(6:, e0158366. doi:10.1371/journal.pone.0158366

Kwan, D., Kurczek, J., \& Rosenbaum, R. S. (2016). Specific, personally meaningful cues can benefit episodic prospection in medial temporal lobe amnesia. British Journal of Clinical Psychology, 55, 137-153. doi:10.1111/bjc.12095

Labouvie-Vief, G., \& Blanchard-Fields, F. (1982). Cognitive ageing and psychological growth. Ageing and Society, 2, 183-209.

Lang, F. R., \& Carstensen, L. L. (2002). Time counts: Future time perspective, goals, and social relationships. Psychology and Aging, 17, 125-139. doi:10.1037/0882-7974.17.1.125

Lamont, R. A., Swift, H. J., \& Abrams, D. (2015). A review and meta-analysis of age-based stereotype threat: Negative stereotypes, not facts, do the damage. Psychology and Aging, 30, 180-193. http://dx.doi.org/10.1037/a0038586 
Lapp, L. K., \& Spaniol, J. (2016). Aging and self-discrepancy: Evidence for adaptive change across the life span. Experimental aging research, 42, 212-219. doi:10.1080/0361073X.2016.1132900

Lapp, L. K., \& Spaniol, J. (2017). Impact of age-relevant goals on future thinking in younger and older adults. Memory, 1-14. doi:10.1080/09658211.2017.1284240

Lardi, C., D'Argembeau, A., Chanal, J., Ghisletta, P., \& Van, d. L. (2010). Further characterisation of self-defining memories in young adults: A study of a Swiss sample. Memory, 18, 293-309. doi:10.1080/09658211003601522

Leitz, J. R., Morgan, C. J. A., Bisby, J. A., Rendell, P. G., \& Curran, H. V. (2009). Global impairment of prospective memory following acute alcohol. Psychopharmacology, 205, 379-387. doi:10.1007/s00213-009-1546-Z

Levine, B., Svoboda, E., Hay, J. F., Moscovitch, M., \& Winocur, G. (2002). Aging and autobiographical memory: Dissociating episodic from semantic retrieval. Psychology and Aging, 17, 677-689.

Lezak, M. D., Howieson, D. B., Bigler, E. D., \& Tranel, D. (2012). Neuropsychological assessment (5th ed.). New York: Oxford University Press.

Lindenberger, U., \& Mayr, U. (2014). Cognitive aging: Is there a dark side to environmental support? Trends in Cognitive Science, 18, 7-15. doi:10.1016/j.tics.2013.10.006

Liu, L. L., \& Park, D. C. (2004). Aging and medical adherence: The use of automatic processes to achieve effortful things. Psychology and Aging, 19, 318-325. doi:10.1037/08827974.19.2.318

Locke, E. A., \& Latham, G. P. (1990). A theory of goal setting and task performance. Englewood Cliffs, NJ: Prentice-Hall. 
Loftus, E.F. (2003). Make-believe memories. American Psychology, 58, 867-873.

Lopez, M. N., Charter, R. A., Mostafavi, B., Nibut, L. P., \& Smith, W. E. (2005). Psychometric properties of the Folstein Mini-Mental State Examination. Assessment, 12, 137-144. doi:10.1177/1073191105275412

Lovibond, P. F., \& Lovibond, S. H. (1995). The structure of negative emotional states: Comparison of the Depression Anxiety Stress Scales (DASS) with the Beck Depression and Anxiety Inventories. Behaviour Research and Therapy 33, 335-343.

Madore, K. P., Gaesser, B., \& Schacter, D. L. (2014). Constructive episodic simulation: Dissociable effects of a specificity induction on remembering, imagining, and describing in young and older adults. Journal of Experimental Psychology: Learning, Memory, and Cognition, 40, 609-622. doi:10.1037/a0034885

Maguire, E.A. (2001). Neuroimaging studies of autobiographical memory. Philosophical Transactions of the Royal Society of London, Series B, 356, 1441-1451. doi:10.1098/rstb.2001.0944

Marks, D. F. (1973). Visual imagery differences in the recall of pictures. British Journal of Psychology, 64, 17-24.

Markus, H., \& Nurius, P. (1986). Possible selves. American Psychologist, 41, 954-969. doi:10.1037/0003-066X.41.9.954

Martin-Ordas, G., Atance, C., \& Louw, A. (2012). The role of episodic and semantic memory in episodic foresight. Learning and Motivation, 43, 209-219.

doi:10.1016/j.lmot.2012.05.011 
Martinelli, P., Anssens, A., Sperduti, M., \& Piolino, P. (2013). The influence of normal aging and Alzheimer's disease in autobiographical memory highly related to the self. Neuropsychology, 27, 69-78. doi:10.1037/a0030453

Masumoto, K., Nishimura, C., Tabuchi, M., \& Fujita, A. (2011). What factors influence prospective memory for elderly people in a naturalistic setting? Japanese Psychological Research, 53, 30-41. doi:10.1111/j.1468-5884.2010.00453.x

Mather, M., \& Carstensen, L. L. (2005). Aging and motivated cognition: The positivity effect in attention and memory. Trends in Cognitive Sciences, 9, 496-502. doi:10.1016/j.tics.2005.08.005

Maylor, E. A. (1990). Age and prospective memory. The Quarterly Journal of Experimental Psychology A: Human Experimental Psychology, 42, 471-493.

McAdams, D. P., Diamond, A., de St. Aubin, E., \& Mansfield, E. (1997). Stories of commitment: The psychosocial construction of generative lives. Journal of Personality and Social Psychology, 72, 678-694. doi:10.1037/0022-3514.72.3.678

McDaniel, M. A., \& Bugg, J. M. (2012). Memory training interventions: What has been forgotten? Journal of Applied Research in Memory and Cognition, 1, 45-50.

McDaniel, M. A., \& Einstein, G. O. (2007). Prospective memory: An overview and synthesis of an emerging field. Thousand Oaks, CA: Sage.

McDaniel, M. A., Einstein, G. O., \& Rendell, P. G. (2008). The puzzle of inconsistent agerelated declines in prospective memory: A multiprocess explanation. Meeting of the Psychonomic Society (pp. 141-160) New York: Erlbaum. 
McDaniel, M. A., \& Einstein, G. O. (2000). Strategic and automatic processes in prospective memory retrieval: A multiprocess framework. Applied Cognitive Psychology, 14, S127S144. doi:10.1002/acp.775

McEvoy, P. M., Erceg-Hurn, D., Saulsman, L. M., \& Thibodeau, M. A. (2015). Imagery enhancements increase the effectiveness of cognitive behavioural group therapy for social anxiety disorder: A benchmarking study. Behaviour Research and Therapy, 65, 42-51. doi:10.1016/j.brat.2014.12.011

McFarland, C. P., \& Glisky, E. L. (2011). Implementation intentions and prospective memory among older adults: An investigation of the role of frontal lobe function. Aging, Neuropsychology, and Cognition, 18(6), 633-652. doi:10.1080/13825585.2011.613449

McFarland, C., \& Glisky, E.L. (2012). Implementation intentions and imagery: Individual and combined effects on prospective memory among young adults. Memory \& Cognition, 40, 62-69. doi:10.3758/s13421-011-0126-8

McKelvie, S. J. (1986). Effects of format of the vividness of visual imagery questionnaire on content validity, split-half reliability, and the role of memory in test-retest reliability. British Journal of Psychology, 77, 229-236. doi:10.1111/j.2044-8295.1986.tb01997.x

Meeks, J. T., \& Marsh, R. L. (2010). Implementation intentions about nonfocal event-based prospective memory tasks. Psychological Research, 74, 82-89. doi:10.1007/s00426-0080223-X

Michaelian, K., Klein, S. B., \& Szpunar, K. K. (Eds.). (2016). Seeing the future: Theoretical perspectives on future-oriented mental time travel. New York: Oxford University Press. doi:10.1093/acprof:oso/9780190241537.001.0001 
Mikels, J. A., Larkin, G. R., Reuter-Lorenz, P., \& Carstensen, L. L. (2005). Divergent trajectories in the aging mind: Changes in working memory for affective versus visual information with age. Psychology and Aging, 20, 542-553. doi:10.1037/08827974.20.4.542

Miloyan, B., Pachana, N. A., \& Suddendorf, T. (2016). Future-oriented thought patterns associated with anxiety and depression in later life: The intriguing prospects of prospection. The Gerontologist, 57. doi:10.1093/geront/gnv695

Mioni, G., Bertucci, E., Rosato, A., Terrett, G., Rendell, P. G., Zamuner, M., \& Stablum, F. (2017). Improving prospective memory performance with future event simulation in traumatic brain injury patients. British Journal of Clinical Psychology, doi:10.1111/bjc. 12126

Mioni, G., Rendell, P. G., Stablum, F., Gamberini, L., \& Bisiacchi, P. S. (2015). Test-retest consistency of virtual week: A task to investigate prospective memory. Neuropsychological Rehabilitation, 25, 419-447. doi:10.1080/09602011.2014.941295

Naveh-Benjamin, M. (2000). Adult age differences in memory performance: Tests of an associative deficit hypothesis. Journal of Experimental Psychology: Learning, Memory, and Cognition, 26, 1170-1187. doi:10.1037/0278-7393.26.5.1170

Neisser, U. (1988). Five kinds of self-knowledge. Philosophical Psychology, 1, 35-59.

Neroni, M. A., Gamboz, N., \& Brandimonte, M. A. (2014). Does episodic future thinking improve prospective remembering? Consciousness and Cognition: An International Journal, 23, 53-62. doi:10.1016/j.concog.2013.12.001 
Osvath, M. \& Osvath, H. (2008). Chimpanzee (Pan troglodytes) and orangutan (Pongo abelii) forethought: self-control and pre-experience in the face of future tool-use. Animal Cognition, 11, 661-674. doi:10.1007/s10071-008-0157-0

Palombo, D. J., Williams, L. J., Abdi, H., \& Levine, B. (2013). The survey of autobiographical memory (SAM): A novel measure of trait mnemonics in everyday life. Cortex: A Journal Devoted to the Study of the Nervous System and Behavior, 49, 1526-1540. doi:10.1016/j.cortex.2012.08.023

Paraskevaides, T., Morgan, C. J. A., Leitz, J. R., Bisby, J. A., Rendell, P. G., \& Curran, H. V. (2010). Drinking and future thinking: Acute effects of alcohol on prospective memory and future simulation. Psychopharmacology, 208, 301-308. doi:10.1007/s00213-009$1731-0$

Pasupathi, M., Lucas, S., \& Coombs, A. (2002). Conversational functions of autobiographical remembering: Long-married couples talk about conflicts and pleasant topics. Discourse Processes, 34, 163-192. doi:10.1207/S15326950DP3402_3

Penningroth, S. L., \& Scott, W. D. (2007). A motivational-cognitive model of prospective memory: The influence of goal relevance. Psychology of motivation. (pp. 115-128) Hauppauge, NY: Nova Science.

Perrin, D., \& Michaelian, K. (2017). Memory as mental time travel. In Bernecker, S., \& Michaelian, K. (Eds.), The Routledge Handbook of Philosophy of Memory (pp. 228-239). Routledge.

Pham, L. B., \& Taylor, S. E. (1999). From thought to action: Effects of process- versus outcomebased mental simulations on performance. Personality and Social Psychology Bulletin, $25,250-260$. 
Phillips, L. H., Henry, J. D., \& Martin, M. (2008). Adult aging and prospective memory: The importance of ecological validity. Prospective memory: Cognitive, neuroscience, developmental, and applied perspectives. (pp. 161-185) New York: Erlbaum.

Pillemer, D. B., Thomsen, D., Kuwabara, K. J., \& Ivcevic, Z. (2013). Feeling good and bad about the past and future self. Memory, 21, 210-218. doi:10.1080/09658211.2012.720263

Piolino, P., Desgranges, B., \& Eustache, F. (2009). Episodic autobiographical memories over the course of time: Cognitive, neuropsychological and neuroimaging findings. Neuropsychologia, 47, 2314-2329. doi:10.1016/j.neuropsychologia.2009.01.020

Piras, F., Borella, E., Incoccia, C., \& Carlesimo, G. A. (2011). Evidence-based practice recommendations for memory rehabilitation. European Journal of Physical and Rehabilitation Medicine, 47, 149-75.

Platt, B., Kamboj, S. K., Italiano, T., Rendell, P. G., \& Curran, H. V. (2016). Prospective memory impairments in heavy social drinkers are partially overcome by future event simulation. Psychopharmacology, 233, 499-506. doi:10.1007/s00213-015-4145-1

Raes, F., Williams, J. M., \& Hermans, D. (2009). Reducing cognitive vulnerability to depression: A preliminary investigation of MEmory Specificity Training (MEST) in inpatients with depressive symptomatology. Journal of Behavior Therapy and Experimental Psychiatry, 40, 24-38. doi:10.1016/j.jbtep.2008.03.001

Rathbone, C. J., Conway, M. A., \& Moulin, C. J. A. (2011). Remembering and imagining: The role of the self. Consciousness and Cognition: An International Journal, 20, 1175-1182. doi:10.1016/j.concog.2011.02.013 
Raz, N., \& Rodrigue, K.M. (2006). Differential aging of the brain: Patterns, cognitive correlates and modifiers. Neuroscience \& Biobehavioral Reviews, 30, 730-748. doi:10.1016/j.neubiorev.2006.07.001

Reed, A. E., Chan, L., \& Mikels, J. A. (2014). Meta-analysis of the age-related positivity effect: Age differences in preferences for positive over negative information. Psychology and Aging, 29, 1-15. doi:10.1037/a0035194

Reichman, W. E., Fiocco, A. J., \& Rose, N. S. (2010). Exercising the brain to avoid cognitive decline: Examining the evidence. Aging Health, 6, 565-584. doi:10.2217/ahe.1054

Reitan, R. M., \& Wolfson, D. (1985). The Halstead-Reitan Neuropsychological Test Battery: Therapy and clinical interpretation. Tucson, AZ: Neuropsychological Press.

Rendell, P. G., \& Craik, F. I. M. (2000). Virtual week and actual week: Age-related differences in prospective memory. Applied Cognitive Psychology, 14, S43-S62. doi:10.1002/acp.770

Rendell, P. G., \& Henry, J. D. (2009). A review of virtual week for prospective memory assessment: Clinical implications. Brain Impairment, 10, 14-22. doi:10.1375/brim.10.1.14

Rendell, P. G., \& Thomson, D. M. (1999). Aging and prospective memory: Differences between naturalistic and laboratory tasks. The Journals of Gerontology: Series B: Psychological Sciences and Social Sciences, 54B, P256-P269.

Reynolds, K., Pietrzak, R. H., El-Gabalawy, R., Mackenzie, C. S., \& Sareen, J. (2015). Prevalence of psychiatric disorders in US older adults: findings from a nationally representative survey. World Psychiatry, 14, 74-81.

Richardson, J. T. (1999). Imagery. East Sussex, UK: Psychology Press, Ltd. 
Rose, N. S., Rendell, P. G., Hering, A., Kliegel, M., Bidelman, G. M., \& Craik, F. I. M. (2015). Cognitive and neural plasticity in older adults' prospective memory following training with the virtual week computer game. Frontiers in Human Neuroscience, 9, 1-13.

Rose, N. S., Rendell, P. G., McDaniel, M. A., Aberle, I., \& Kliegel, M. (2010). Age and individual differences in prospective memory during a "virtual week": The roles of working memory, vigilance, task regularity, and cue focality. Psychology and Aging, 25, 595-605. doi:10.1037/a0019771

Rosenbaum, R. S., Köhler, S., Schacter, D. L., Moscovitch, M., Westmacott, R., Black, S. E., .. . Tulving, E. (2005). The case of K.C.: Contributions of a memory-impaired person to memory theory. Neuropsychologia, 43, 989-1021.

doi:10.1016/j.neuropsychologia.2004.10.007

Rubin, D. C., Berntsen, D., \& Hutson, M. (2009). The normative and the personal life: Individual differences in life scripts and life story events among USA and danish undergraduates. Memory, 17, 54-68. doi:10.1080/09658210802541442

Schacter, D. L. (2012). Adaptive constructive processes and the future of memory. American Psychologist, 67, 603-613. doi:10.1037/a0029869

Schacter, D.L. \& Addis, D.R. (2007). The cognitive neuroscience of constructive memory: Remembering the past and imagining the future. Philosophical Transactions of the Royal Society B: Biological Sciences, 362, 773-786. doi:10.1098/rstb.2007.2087

Schacter, D. L., Chamberlain, J., Gaesser, B., \& Gerlach, K. D. (2012). Neuroimaging of true, false, and imaginary memories: Findings and implications. Memory and law. (pp. 233262) New York: Oxford University Press. 
Schacter, D. L., Gaesser, B., \& Addis, D. R. (2013). Remembering the past and imagining the future in the elderly. Gerontology,59(2), 143-151. doi:10.1159/000342198

Schneider, B.A., Avivi-Reich, M., \& Mozuraitis, M. (2015). A cautionary note on the use of the analysis of covariance (ANCOVA) in classification designs with and without withinsubject factors. Frontiers in Psychology, 6, 1-12. doi:10.3389/fpsyg.2015.00474

Schnitzspahn, K. M., Ihle, A., Henry, J. D., Rendell, P. G., \& Kliegel, M. (2011). The ageprospective memory-paradox: An exploration of possible mechanisms. International Psychogeriatrics, 23, 583-592. doi:10.1017/S1041610210001651

Schnitzspahn, K. M., \& Kliegel, M. (2009). Age effects in prospective memory performance within older adults: The paradoxical impact of implementation intentions. European Journal of Ageing, 6, 147-155. doi:10.1007/s10433-009-0116-х

Seligman, M. E., Railton, P., Baumeister, R. F., \& Sripada, C. (2016). Homo prospectus. New York: Oxford University Press.

Seligman, M. E. P., Railton, P., Baumeister, R. F., \& Sripada, C. (2013). Navigating into the future or driven by the past. Perspectives on Psychological Science, 8, 119-141. doi:10.1177/1745691612474317

Sellers, A. H., \& Nadler, J. D. (1992). A survey of current neuropsychological assessment procedures used for different age groups. Psychotherapy in Private Practice, 11, 47-57. doi:10.1300/J294v11n03_10

Sheldon, S., \& Chu, S. (2017). What versus where: Investigating how autobiographical memory retrieval differs when accessed with thematic versus spatial information. The Quarterly Journal of Experimental Psychology, 70, 1909-1921.

doi:10.1080/17470218.2016.1215478 
Sheldon, S., Farb, N., Palombo, D. J., \& Levine, B. (2016). Intrinsic medial temporal lobe connectivity relates to individual differences in episodic autobiographical remembering. Cortex: A Journal Devoted to the Study of the Nervous System and Behavior, 74, 206216. doi:10.1016/j.cortex.2015.11.005

Sheldon, S., McAndrews, M. P., \& Moscovitch, M. (2011). Episodic memory processes mediated by the medial temporal lobes contribute to open-ended problem solving. Neuropsychologia, 49, 2439-2447. doi:10.1016/j.neuropsychologia.2011.04.021

Shipley, W. C. (1940). A self-administering scale for measuring intellectual impairment and deterioration. Journal of Psychology: Interdisciplinary and Applied, 9, 371-377. doi:10.1080/00223980.1940.9917704

Simons, J. S., Schölvinck, M. L., Gilbert, S. J., Frith, C. D., \& Burgess, P. W. (2006). Differential components of prospective memory? Evidence from fMRI. Neuropsychologia, 44, 1388-1397. doi:10.1016/j.neuropsychologia.2006.01.005

Singer, J. A., \& Blagov, P. (2004). The integrative function of narrative processing: Autobiographical memory, self-defining memories, and the life story of identity. In D. R. Beike, J. M. Lampinen \& D. A. Behrend (Eds.), The self and memory; The self and memory (pp. 117-138) New York: Psychology Press.

Singer, J., Rexhaj, B., \& Baddeley, J. (2007). Older, wiser, and happier? Comparing older adults' and college students' self-defining memories. Memory, 15, 886-898. doi:10.1080/09658210701754351

Smith, J., \& Freund, A. M. (2002). The dynamics of possible selves in old age. The Journals of Gerontology: Series B: Psychological Sciences and Social Sciences, 57B, P492-P500. doi:10.1093/geronb/57.6.P492 
Smith, B. T., \& Hess, T. M. (2015). The impact of motivation and task difficulty on resource engagement: Differential influences on cardiovascular responses of young and older adults. Motivation Science, 1, 22-36. doi:10.1037/mot0000012

Smith, G., Sala, S. D., Logie, R. H., \& Maylor, E. A. (2000). Prospective and retrospective memory in normal ageing and dementia: A questionnaire study. Memory, 8, 311-321.

Spaniol, J., Schain, C., \& Bowen, H. J. (2014). Reward-enhanced memory in younger and older adults. The Journals of Gerontology: Series B: Psychological Sciences and Social Sciences, 69B, 730-740. doi:10.1093/geronb/gbt044

Spaniol, J., Voss, A., Bowen, H. J., \& Grady, C. L. (2011). Motivational incentives modulate age differences in visual perception. Psychology and Aging, 26, 932-939. doi:10.1037/a0023297

Spreng, R. N., \& Levine, B. (2013). Doing what we imagine: Completion rates and frequency attributes of imagined future events one year after prospection. Memory, 21, 458-466. doi:10.1080/09658211.2012.736524

Spreng, R. N., \& Levine, B. (2006). The temporal distribution of past and future autobiographical events across the lifespan.Memory \& Cognition, 34, 1644-1651. doi:10.3758/BF03195927

Squire, L. R. (2004). Memory systems of the brain: A brief history and current perspective. Neurobiology of Learning and Memory, 82, 171-177. doi:10.1016/j.nlm.2004.06.005

Stawarczyk, D., \& D'Argembeau, A. (2015). Neural correlates of personal goal processing during episodic future thinking and mind-wandering: An ALE meta-analysis. Human Brain Mapping, 36, 2928-2947. doi:10.1002/hbm.22818 
Stokes, M., Thompson, R., Cusack, R., \& Duncan, J. (2009). Top-down activation of shapespecific population codes in visual cortex during mental imagery. The Journal of Neuroscience, 29, 1565-1572. doi:10.1523/JNEUROSCI.4657-08.2009

Strauss, E., Sherman, E. M., \& Spreen, O. (2006). A compendium of neuropsychological tests: Administration, norms, and commentary. New York: Oxford University Press.

Stuss, D. T., Stethem, L. L., \& Poirier, C. A. (1987). Comparison of three tests of attention and rapid information processing across six age groups. The Clinical Neuropsychologist, 1,

139-152. doi:10.1080/13854048708520046

Suddendorf, T., \& Corballis, M. C. (1997). Mental time travel and the evolution of the human mind. Genetic, Social, and General Psychology Monographs, 123, 133-167.

Szpunar, K. K. (2010). Episodic future thought: An emerging concept. Perspectives on Psychological Science, 5(2), 142-162. doi:10.1177/1745691610362350

Szpunar, K.Z., Spreng, N.R., \& Schacter, D.L. (2014). A taxonomy of prospection: Introducing organizational framework for future-oriented cognition. Proceedings of the National Academy of Sciences, 111, 1-8. doi:10.1073/pnas.1417144111

Taylor, S. E., Pham, L. B., Rivkin, I. D., \& Armor, D. A. (1998). Harnessing the imagination: Mental simulation, self-regulation, and coping. American Psychologist, 53, 429-439. doi:10.1037/0003-066X.53.4.429

Teasdale, J. D., Segal, Z. V., Williams, J. M., Ridgeway, V. A., Soulsby, J. M., \& Lau, M. A. (2000). Prevention of relapse/recurrence in major depression by mindfulness-based cognitive therapy. Journal of Consulting and Clinical Psychology, 68, 615-623. doi:10.1037/0022-006X.68.4.615 
Terrett, G., Rose, N. S., Henry, J. D., Bailey, P. E., Altgassen, M., Phillips, L. H., . . Rendell, P. G. (2016). The relationship between prospective memory and episodic future thinking in younger and older adulthood. The Quarterly Journal of Experimental Psychology, 69, 310-323. doi:10.1080/17470218.2015.1054294

Terry, W. S. (1988). Everyday forgetting: Data from a diary study. Psychological Reports, 62, 299-303.

Tombaugh, T. N. (2004). Trail Making Test A and B: Normative data stratified by age and education. Archives of Clinical Neuropsychology, 19, 203-214. doi:10.1016/S08876177(03)00039-8

Tombaugh, T. N., Kozak, J., \& Rees, L. (1999). Normative data stratified by age and education for two measures of verbal fluency: FAS and animal naming. Archives of Clinical Neuropsychology, 14, 167-177.

Tombaugh, T. N., \& McIntyre, N. J. (1992). The Mini-Mental State Examination: A comprehensive review. Journal of the American Geriatrics Society, 40, 922-935. doi:10.1111/j.1532-5415.1992.tb01992.x

Trope, Y., \& Liberman, N. (2003). Temporal construal. Psychological Review, 110, 403-421. doi:10.1037/0033-295X.110.3.403

Trunk, D. L., \& Abrams, L. (2009). Do younger and older adults' communicative goals influence off-topic speech in autobiographical narratives? Psychology and Aging, 24, 324-337. doi:10.1037/a0015259

Tulving, E. (1983). Elements of episodic memory (Vol. 2). New York: Oxford University Press. Tulving, E. (1985). Memory and consciousness. Canadian Psychology/Psychologie Canadienne, 26, 1-12. doi: $10.1037 / \mathrm{h} 0080017$ 
Tulving, E., \& Thomson, D. M. (1973). Encoding specificity and retrieval processes in episodic memory. Psychological Review, 80, 352-373. doi:10.1037/h0020071

Uttl, B. (2011). Transparent meta-analysis: Does aging spare prospective memory with focal vs. non focal cues? PLoS ONE,6. doi:10.1371/journal.pone.0016618

Velleman, P.F., \& Wilkinson, L. (1993). Nominal, ordinal, interval, and ratio typologies are misleading. The American Statistician, 47, 65-72.

Viard, A., Chetelat, G., Lebreton, K., Desgranges, B., Landeau, B., Sayette, V., Eustach, F., \& Piolino, P. (2011). Mental time travel into the past and the future in healthy aged adults: An fMRI study. Brain and Cognition, 75, 1-9. doi:10.1016/j.bandc.2010.10.009.

Walters, S., \& Meier, B. (2014). How important is importance for prospective memory? A review. Frontiers in Psychology, 5, 1-9.

Wang, Q. (2016). Remembering the self in cultural contexts: A cultural dynamic theory of autobiographical memory. Memory Studies, 9, 295-304. doi.10.1177/1750698016645238

Ward, A. M. (2016). A critical evaluation of the validity of episodic future thinking: A clinical neuropsychology perspective. Neuropsychology, 30, 887-905. doi:10.1037/neu0000274

Watson, D., Clark, L. A., \& Tellegen, A. (1988). Development and validation of brief measures of positive and negative affect: The PANAS scales. Journal of Personality and Social Psychology, 54, 1063-1070. doi:10.1037/0022-3514.54.6.1063

Wechsler, D. (2009). Advanced clinical solutions for the WAIS-IV and WMS-IV. San Antonio: Pearson.

West, R. L. (1988). Prospective memory and aging. In M. M. Grunebberg, P. E. Morris, \&R. N.Sykes (Eds.), Practical aspects of memory: Current research and issues: Vol. 2. Clinical and educational implications (pp. 119-125). New York: Wiley. 
Wheeler, M. A., Stuss, D. T., \& Tulving, E. (1997). Toward a theory of episodic memory: The frontal lobes and autonoetic consciousness. Psychological Bulletin, 121, 331-354. doi:10.1037/0033-2909.121.3.331

Williams, J. M. (2006). Capture and rumination, functional avoidance, and executive control (CaRFAX): Three processes that underlie overgeneral memory. Cognition and Emotion, 20, 548-568. doi:10.1080/02699930500450465

Zacks, R. T., Hasher, L., \& Li, K. Z. H. (2000). Human memory. In Craik F. I. M., Salthouse T. A. (Eds.), The Handbook of Cognitive Aging, $2^{\text {nd }}$ Edition (pp. 293-357). Mahwah, NJ: Erlbaum.

Zimmermann, T. D., \& Meier, B. (2010). The effect of implementation intentions on prospective memory performance across the lifespan. Applied Cognitive Psychology, 24, 645-658. doi:10.1002/acp.1576 